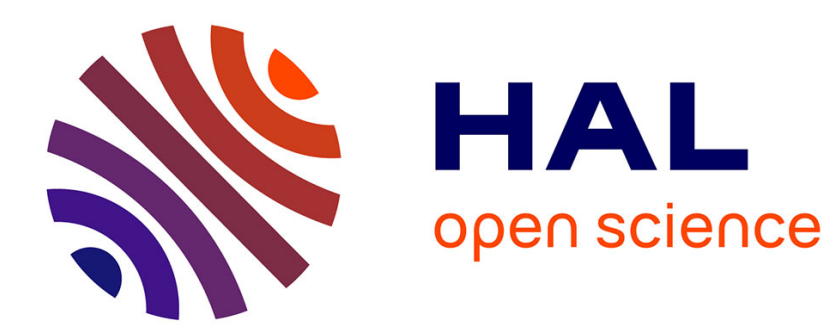

\title{
Les mineurs non accompagnés : au croisement des rôles sociaux
}

Amélie Thomas

\section{To cite this version:}

Amélie Thomas. Les mineurs non accompagnés: au croisement des rôles sociaux. Sociologie. 2019. dumas-03359536

\section{HAL Id: dumas-03359536 https://dumas.ccsd.cnrs.fr/dumas-03359536}

Submitted on 30 Sep 2021

HAL is a multi-disciplinary open access archive for the deposit and dissemination of scientific research documents, whether they are published or not. The documents may come from teaching and research institutions in France or abroad, or from public or private research centers.
L'archive ouverte pluridisciplinaire HAL, est destinée au dépôt et à la diffusion de documents scientifiques de niveau recherche, publiés ou non, émanant des établissements d'enseignement et de recherche français ou étrangers, des laboratoires publics ou privés. 


\title{
Université $/$ Faculté de sociologie
}

Master 2 Sciences Sociales

Parcours Intervention et Innovation Sociales

\section{LES MINEURS NON ACCOMPAGNÉS \\ AU CROISEMENT DES RÔLES SOCIAUX}

\author{
Mémoire présenté par Amélie THOMAS
}

Soutenu le 03/10/2019

Sous la direction de Claire SCHIFF

Maîtresse de conférences en sociologie

Jury composé de Claire SCHIFF, Agnès VILLECHAISE, Hervé BRUN 


\section{LES MINEURS NON ACCOMPAGNÉS AU CROISEMENT DES RÔLES SOCIAUX}


Université de Bordeaux

Faculté de Sociologie

Master 2 Sciences Sociales

Parcours Intervention et Innovation Sociales

\section{LES MINEURS NON ACCOMPAGNÉS AU CROISEMENT DES RÔLES SOCIAUX}

Mémoire présenté par Amélie THOMAS

Soutenu le 03/10/2019

Sous la direction de Claire SCHIFF

Maîtresse de conférences en sociologie

Jury composé de Claire SCHIFF, Agnès VILLECHAISE, Hervé BRUN 
Claire SCHIFF

Agnès VILLECHAISE

Hervé BRUN

Marine PISANI

Les relecteurs

Les collègues de promotion

Les collègues du Service de Réadaptation Sociale pour Adolescents

L'équipe du Service d'Accueil et d'Évaluation des Mineurs Non Accompagnés

L'équipe du Carré d'Accueil pour les Mineurs Non Accompagnés

L'équipe de $\mathrm{R} d$ 'accueil

Les bénévoles du Squid

Les jeunes qui ont participé

Ceux que j'accompagne... 


\section{PRÉAMBULE}

Un appartement institutionnel, un déménagement...

L'approche des 21 ans et la fin de prise en charge en perspective...

- Je ne sais même pas qui tu es !

- Bah si tu sais, je m'appelle Redei D.

- Redei $D, c^{\prime}$ est ton vrai nom ?

- Oui.

- Et tu es né le 8 juin 1997 ?

- 1993. Je vais avoir 25 ans.

Après un silence...

- Ça te fait quoi d'avoir deux histoires?

- Je sais qui je suis... C'est là, dans ma tête !

Un épisode comme en suspens dans le suivi éducatif... 


\section{RÉSUMÉ}

Dans le contexte actuel de "crise migratoire », les Mineurs Non Accompagnés arrivent en nombre dans les services de protection de l'enfance, venant ainsi interroger les pratiques professionnelles. L'étude donne la parole à ces jeunes afin de recueillir leurs vécus de la situation post-migratoire. La réflexion porte sur les expériences qu'ils font de la rencontre avec la société d'accueil au regard de leurs projets de départ et des différents rôles qui leur sont attribués là-bas et ici. Trente-trois entretiens ont été menés à différentes étapes du parcours au sein de l'Aide Sociale à l'Enfance, allant de l'évaluation de la minorité à la sortie du dispositif. II s'est agi d'explorer les trajectoires entre deux cultures, mais aussi entre deux âges, dans une dialectique entre l'individuel et le collectif. En filigrane est ressortie la question du statut. La recherche montre que tour à tour agents et acteurs, les Mineurs Non Accompagnés s'inscrivent dans un processus d'adaptation à la société d'accueil et voient la réalisation de leurs projets facilitée par les exigences des dispositifs d'accompagnement. Recueillir la subjectivité des acteurs sous l'angle de l'interculturalité confère à l'étude une dimension anthropologique.

\section{MOTS CLÉS}

Acteur / agent - autonomie - expérience - individuation - jeunesse - Mineurs Non Accompagnés - processus d'adaptation - projet migratoire -rôle social - statut. 


\title{
INTRODUCTION
}

\section{PARTIE 1. UNE MIGRATION AUX CONTOURS PARTICULIERS}

1. Une expérience nouvelle de la migration

2. Tantôt acteur, tantôt agent du projet migratoire

3. Prendre la route ou la suivre

\section{PARTIE 2. RENCONTRE AVEC UNE SOCIETE D'ACCUEIL EXIGEANTE}

1. Des droits et des démarches

2. Des attentes envers l'aide sociale à l'enfance évolutives

3. Exigences de régularisation et d'autonomisation

\section{PARTIE 3. UNE JEUNESSE DANS L'ENTRE-DEUX}

1. Entre deux âges, entre deux cultures

2. Des projets et des acteurs

3. Adaptation, intégration et individuation

\section{CONCLUSION}

SOURCES ET RÉFÉRENCES BIBLIOGRAPHIQUES

\author{
TABLE DES MATIÈRES
}

\section{ANNEXES}




\section{INTRODUCTION}

\section{LES MINEURS NON ACCOMPAGNÉS : ACTUALITÉ ET CONTEXTE LEGISLATIF}

En 2015 s'impose dans les médias l'expression « crise migratoire " en référence à "l'afflux » de migrants arrivant aux frontières européennes, du fait des conflits aux Proche et Moyen-Orient principalement. Si le nombre de demandes d'asile augmente significativement cette année-là, la question migratoire était déjà centrale depuis de nombreuses décennies, du fait de l'écart de développement entre l'Europe et d'autres régions du monde. Les "printemps arabes » de 2011 avaient par ailleurs généré une émigration massive qui s'était ajoutée au déplacement des populations d'Afrique subsaharienne fuyant les guerres, les dictatures ou encore la précarité économique. En parallèle à ces migrations d'adultes et de familles, augmente le nombre de mineurs arrivant seuls. Cette catégorie de migrants est apparue en Europe dès le début des années 1990, principalement en Allemagne, au Royaume-Uni, en Belgique et en France, puis quelques années plus tard, en Italie et en Espagne, en provenance du Maghreb et de l'Afrique de l'Ouest (Blanchard et Rodier, 2016).

Très rapidement, le constat est fait d'un vide juridique pour encadrer l'accueil de ces mineurs étrangers. Chaque État membre de l'Union européenne improvise des réponses allant de l'inclusion dans le droit commun à la mise en place de législations spécifiques. En 2014, Daniel Senovilla-Hernández se penche sur la question et retrace l'évolution de la prise en compte de cette catégorie juridique spécifique en Europe. II relève qu'en 1997, le Conseil de l'Union européenne propose un assouplissement des règles en matière d'immigration en direction des "mineurs non accompagnés ressortissants de pays tiers ". Par ailleurs, il est spécifié dans l'article 2 de la CIDE $^{1}$ ratifiée le 20 novembre 1989 qu'elle s'applique à tous les mineurs, sans distinction aucune. Cependant, son interprétation concernant l'intérêt supérieur de l'enfant reste à l'appréciation de chaque État ${ }^{2}$.

Ce n'est qu'en 2005 que le Comité des droits de l'enfant des Nations Unies énonce des principes de traitement des "mineurs étrangers non accompagnés ". Cette terminologie fait référence à tout « enfant de moins de dix-huit ans qui a été séparé de ses deux parents et d'autres membres proches de sa famille et n'est pas pris en charge par un adulte investi de cette responsabilité par la loi et la coutume ». La notion de "mineur séparé » vient compléter la précédente en désignant un enfant « qui a été séparé de ses deux parents ou des personnes qui en avaient la charge à

\footnotetext{
${ }^{1}$ Convention Internationale des Droits de l'Enfant.

${ }^{2} \mathrm{Cf}$. annexe 1 p.l.
} 
titre principal auparavant en vertu de la loi ou de la coutume, mais pas nécessairement d'autres membres de sa famille. Un enfant séparé peut donc être accompagné par un autre membre adulte de sa famille. " Durant de nombreuses années, aucun terme commun n'existe en Europe pour désigner cette catégorie de migrants. Pourtant, dans la circulaire du Parlement européen et du Conseil du 13 décembre 2011 est posée la notion de "Mineur Non Accompagné " définie comme " un mineur qui entre sur le territoire des États membres sans être accompagné d'un adulte qui est responsable de lui, de par le droit ou la pratique en vigueur dans l'État membre concerné, et tant qu'il n'est pas effectivement pris en charge par une telle personne ; cette expression couvre aussi le mineur qui a été laissé seul après être entré sur le territoire des États membres ${ }^{3}$. " En France, on utilise l'expression "Mineur Isolé Étranger ", jusqu'à ce que la garde des Sceaux institue lors du comité de suivi du 7 mars 2016 le recours à la dénomination de "Mineur Non Accompagné " pour être en adéquation avec la directive européenne énoncée précédemment, mais aussi afin de mettre l'accent sur l'isolement plutôt que sur l'extranéité des jeunes concernés.

L'étude comparative réalisée en 2010 par Daniel Senovilla-Hernández met en avant l'existence de trois modèles différents régissant en Europe l'accueil de ces mineurs. Certains pays comme l'Allemagne et le Royaume-Uni n'accordent le bénéfice de la protection qu'à ceux qui effectuent une demande d'asile. D'autres, tels que I'Italie et l'Espagne, n'excluent aucune catégorie de mineurs mais ne distinguent nullement leur migration de celle des adultes venant pour des raisons économiques et leurs pratiques administratives en matière de régularisation sont arbitraires, avec la mise en place de mesures de rapatriement. Le troisième modèle est celui appliqué en France et en Belgique, où l'on considère les mineurs étrangers comme étant en danger et devant être protégés, mais où s'opère un filtrage à la frontière avec détention et retours possibles dans les pays d'origine.

Dans un contexte de crise économique et de gestion des flux migratoires, les États adoptent des stratégies visant à exclure des dispositifs de protection de l'enfance certains mineurs étrangers, pratique qui va à l'encontre de la $\operatorname{CIDE}^{4}$. Quelle que soit l'expression retenue pour désigner cette catégorie juridique, elle revêt trois éléments que sont la minorité d'âge, la condition d'étranger et enfin, la potentielle situation de danger liée à l'absence de représentants légaux. Filtrer l'accès aux services d'assistance éducative consiste à mettre en doute l'appartenance à cette catégorie et s'opère dans la première phase d'identification, qui précède la prise en charge et la recherche de solutions durables. Il peut s'agir de nier la condition d'isolement en officialisant

\footnotetext{
${ }^{3} \mathrm{Cf}$. annexe 1 p.l.

${ }^{4}$ Convention Internationale des Droits de l'Enfant.
} 
l'accueil chez des proches ou en rapatriant l'enfant vers son pays d'origine, sans que soit forcément pris en compte son intérêt supérieur. Une autre pratique porte sur la contestation de la minorité.

$L^{\prime}$ article 20 de la $\operatorname{CIDE}^{5}$ du 20 novembre 1989 pose le principe de la protection par l'État de tout enfant privé de son milieu familial ou pour qui un éloignement est dans son intérêt. En France, la loi du 5 mars 2007 réformant la protection de l'enfance stipule que les Mineurs Non Accompagnés relèvent des services de protection de l'enfance. La loi du 14 mars 2016 relative à la protection de l'enfant précise que le lieu de placement est décidé dans l'intérêt du mineur. Elle donne lieu à la mise en place du dispositif de répartition des Mineurs Non Accompagnés entre les départements. Le décret en Conseil d'État du 24 juin 2016 fixe les conditions d'accueil, d'évaluation et d'orientation. Le président du Conseil Départemental statue sur la reconnaissance ou non de la qualité de mineur privé temporairement ou définitivement de la protection de sa famille à partir du rapport de synthèse et de l'avis du service chargé de l'évaluation. En cas de remise en question de l'authenticité des documents, le préfet peut être interpellé. Si un doute existe sur l'âge, l'autorité judiciaire est saisie. Au sein de la SDMPJE ${ }^{6}$, la $\mathrm{MMNA}^{7}$ coordonne le dispositif national de mise à l'abri, $d^{\prime}$ évaluation et d'orientation des Mineurs Non Accompagnés ${ }^{8}$.

La presse écrite et les rapports nationaux donnent à partir de 2014 des chiffres portant sur la migration des mineurs. Des données statistiques néanmoins incertaines, d'autant qu'elles laissent de côté les personnes pour qui la minorité a été contestée. Par ailleurs, il n'est pas précisé si sont pris en compte ou non les Mineurs Non Accompagnés devenus majeurs et toujours pris en charge dans le dispositif. Nous pouvons supposer qu'ils sont exclus de la comptabilisation et pourtant, bien qu'ayant accédé à la majorité, notre intérêt porte aussi sur eux, du moins dans cette étude.

Estimés à 2500 en 2004 sur le territoire français, les Mineurs Non Accompagnés étaient entre 4000 et 8000 en 2010. La MMNA créée en 2013 au sein de la DPJ ${ }^{9}$ effectue dorénavant un recueil de données plus précis auprès des Conseils Départementaux. On peut retrouver une partie de ces statistiques dans le rapport du Sénat du 28 juin 2017, dit rapport Doineau. Le nombre de Mineurs Non Accompagnés pris en charge par le dispositif de l'Aide Sociale à l'Enfance est passé de 10194 en 2015 à 13008 en 2016 et 21013 en 2017 . Alors que 8054 nouvelles personnes étaient reconnues mineures en 2016, ce chiffre passait à 14908 en 2017 et pour l'année 2018,

\footnotetext{
${ }^{5}$ Convention Internationale des Droits de l'Enfant.

${ }^{6}$ Sous-Direction des Missions de Protection Judiciaire et d'Éducation.

${ }^{7}$ Mission Mineurs Non Accompagnés.

${ }^{8} \mathrm{Cf}$. annexe 1 p.l.

${ }^{9}$ Direction de la Protection Judiciaire de la Jeunesse.
} 
il était déjà de 10424 au 24 août. Au 17 mai 2019, 8103 nouvelles ordonnances de placement ont été délivrées. On estime à 25000 le nombre de Mineurs Non Accompagnés qui seront accueillis dans les services de l'Aide Sociale à l'Enfance à la fin de l'année.

En Gironde, l'augmentation des prises en charge est tout aussi significative qu'au niveau national. D'après la presse locale, mais aussi les informations transmises par le Conseil Départemental, elles seraient passées de 200 en 2015 à 529 en 2017 (dont 387 nouveaux accueils pour cette seule année). En 2018, 361 ordonnances de placement ont été prononcées. On dénombrait 560 Mineurs Non Accompagnés en Gironde en mars 2018, 1000 début 2019. Au 17 mai 2019, la MMNA ${ }^{10}$ du ministère de la justice enregistrait 188 saisines depuis le 1er janvier. Le département attendait 400 arrivées au cours de l'année 2019.

Nous avons vu que la prise en charge des Mineurs Non Accompagnés en France relevait des services départementaux de l'Aide Sociale à l'Enfance, au même titre que pour tout enfant se trouvant en situation de danger. L'arrivée massive ces dernières années de ces publics, dans le contexte de "crise migratoire " que nous avons décrit brièvement, a inévitablement impacté le dispositif d'accompagnement. En Gironde, pour faire face aux besoins d'accueil aussi bien en direct que dans le cadre de la péréquation, des places supplémentaires sont ouvertes au sein des établissements existants, en parallèle à l'émergence de nouveaux dispositifs, innovants pour certains. De nouveaux postes sont aussi créés (assistants sociaux, juristes, coordinateurs infirmiers, ou encore chargés de missions).

\section{UN PUBLIC SPÉCIFIQUE FAISANT ÉMERGER DE NOUVELLES INTERROGATIONS}

Avec l'arrivée massive des Mineurs Non Accompagnés dans le dispositif, des réajustements sont opérés et de nombreux questionnements émergent. Au niveau éducatif, les professionnels expérimentés du secteur de la protection de l'enfance se voient interpellés, remués, voire déstabilisés par les spécificités de ce public et ses besoins particuliers. Dans d'autres institutions plus spécialisées, les compétences techniques prévalent, au risque parfois d'en oublier le statut d'enfant en danger au profit de celui de migrant. Il s'agit pour les équipes éducatives de se former aux spécificités des Mineurs Non Accompagnés tout en veillant à conserver une approche globale.

Pour encadrer les démarches administratives, le suivi médical ou encore l'accès à la formation, qui nécessitent une attention particulière, des partenariats sont développés et des outils élaborés. Mais qu'en est-il de la relation éducative en ellemême ? Que se joue-t-il entre les jeunes et les éducateurs ? Quelles interrogations

\footnotetext{
${ }^{10}$ Mission Mineurs Non Accompagnés.
} 
sont soulevées ? A quels obstacles se heurtent les intervenants? II semble que de nouvelles grilles de lecture soient à adopter pour appréhender les besoins des Mineurs Non Accompagnés. Ainsi, lorsqu'un jeune effectue des demandes financières incessantes, point de crispation récurrent dans les suivis, n'est-il pas nécessaire d'en comprendre les enjeux? Quelles attentes les Mineurs Non Accompagnés ont-ils des services éducatifs? Par ailleurs, le travail de l'éducateur repose sur la relation, supposée de confiance, qu'établit avec lui la personne accompagnée. Quid de cette relation lorsqu'un décalage existe potentiellement entre l'histoire de vie et le récit qui en est fait, entre l'identité réelle et celle figurant sur les documents administratifs ? Dans ce contexte, comment travailler avec un jeune sa place dans la société, son rapport aux autres?

$\mathrm{Au}$ début de ce travail de recherche, nous partions du constat que des décalages existaient entre les représentations des Mineurs Non Accompagnés et les modalités de prise en charge proposées. Nous envisagions alors de mener une étude comparative auprès des jeunes d'une part, des équipes de l'autre, l'objectif étant de mieux identifier les besoins, mais aussi de repérer les points de blocage, en vue d'améliorer les modes d'accompagnement. Nous avons pourtant écarté l'approche centrée sur les équipes pour deux raisons. Premièrement par crainte d'ouvrir la porte aux doléances et de ne pas pouvoir nous décaler des thématiques récurrentes concernant ce public, telles que l'argent, la scolarisation, les démarches administratives ou encore la santé. Deuxièmement parce qu'il nous semblait pertinent de nous tourner vers les jeunes à qui nous avons peu l'occasion de donner la parole. Nous avons souhaité également nous décentrer de l'évaluation des dispositifs d'accompagnement, de nombreux rapports existant déjà sur ce sujet, émanant d'organismes dédiés (nous en évoquerons brièvement quelques-uns).

Nous nous sommes alors demandé quels étaient les vécus de ces jeunes en situation post-migratoire au regard de leurs aspirations initiales. En quoi les projets de départ des Mineurs Non Accompagnés évoluent-ils au contact de la société d'accueil ? C'est donc l'expression utilisée dans la circulaire européenne de 2011 énoncée précédemment et dont l'usage a été depuis généralisé en France, que nous avons retenue pour désigner le public cible de l'étude. Bien que le premier aspect de cette catégorie juridique renvoie à un critère d'âge, nous utiliserons le terme de Mineur Non Accompagné en référence aussi bien aux mineurs qu'aux jeunes majeurs. Cet abus de langage prend effectivement sens du fait que la recherche porte sur l'ensemble de ces publics, l'accès à la majorité constituant une continuité dans les parcours, avec de nouveaux enjeux que nous interrogerons. En nous penchant sur le "vécu " de ces jeunes, nous nous inspirons de l'approche anthropologique centrée sur la subjectivité 
des acteurs: partir ainsi de ressentis individuels pour tenter de dégager des tendances ; sortir des a priori et des visions stéréotypées qui mettent de côté la parole des principaux intéressés. Quant à la situation post-migratoire, elle renvoie à une étape spécifique des parcours que Joël Fronteau s'attelle à expliciter (dans Legault, 2000), en articulation avec les contextes pré-migratoires et migratoires. Les personnes auxquelles nous consacrons cette recherche se trouvent généralement dans la phase dite "d'adaptation ", entre l'imaginaire et le réel. Ces deux derniers concepts sont directement en lien avec ce que nous nommons les projets de départ et la rencontre avec la société d'accueil. "L'ailleurs fantasmé », que décrit Thomas Fouquet (2007) et qui est souvent à l'origine des migrations, se confronte à l'épreuve de la réalité, dans ses différents aspects. Le sujet doit se conformer aux attentes existant à son égard làbas et ici. Les attentes, mais aussi les perceptions qu'il en a, renvoient au concept de "rôle social ", repris par Jacques Coenen-Huther (2005). C'est la notion que nous retiendrons pour interroger la façon dont les Mineurs Non Accompagnés se positionnent et agissent. Notre problématique de recherche sera la suivante : quelles expériences font les Mineurs Non Accompagnés de la rencontre avec la société d'accueil au regard de leurs projets de départ et des différents rôles qui leur sont attribués là-bas et ici?

En premier lieu, il s'agira de comprendre qui sont ces Mineurs Non Accompagnés. Et pour cela, un détour par les contextes de départ est incontournable. Comment vivaient-ils dans leurs pays d'origine et qu'est-ce qui les a poussés à partir ? Quels rôles leur sont attribués au sein de leurs familles? Quelles représentations avaient-ils de l'Europe et de la France ? Que venaient-ils chercher ici ? II conviendra ensuite d'interroger la rencontre avec la société d'accueil et de quelle manière elle fait évoluer leurs projets. Quelles attentes pèsent sur eux dans la société française ? Comment vivent-ils le dispositif de l'Aide Sociale à l'Enfance? En avaient-ils connaissance avant leur arrivée et si c'est le cas, qu'en espéraient-ils ? Quels effets ont sur eux les exigences liées au droit au séjour? Nous comparerons les rôles sociaux attribués en fonction de l'âge des jeunes, mais aussi au fil de leurs parcours au sein du dispositif de l'Aide Sociale à l'Enfance. Enfin, nous nous intéresserons à la manière dont les Mineurs Non Accompagnés s'adaptent à leur nouvel environnement. Quels sont leurs modes de socialisation et sur qui s'appuient-ils ? Dans la continuité de ces différentes interrogations, nous nous demanderons ce que signifie pour eux " être jeunes » et de quelle manière ils vivent le passage à l'âge adulte dans ce contexte postmigratoire. Comment se pensent-ils en tant que futurs adultes entre là-bas et ici ?

Face à ces questionnements, nous émettons plusieurs hypothèses fondées sur les observations de terrain dans le cadre de l'accompagnement de ces publics. La première est que la société d'accueil présente des exigences en décalage avec les 
représentations et les attentes des Mineurs Non Accompagnés, tout en favorisant la réalisation de leurs projets. La seconde est que ces jeunes adoptent des stratégies d'action pouvant menacer leur " identité intégratrice ». Cette notion, empruntée ici à François Dubet (1994), renvoie au vécu subjectif de l'intégration à une organisation sociale, à l'intériorisation de rôles à travers la socialisation primaire, aux assignations sociales, à la personnalité de la base, en somme. Elle désigne les appartenances, intégrant le Moi au Nous. "Aucun de nous n'échappe à cette forme d'identification à travers la filiation, un nom, le fantasme d'une tradition familiale, l'attachement à quelques valeurs si profondément enfouies qu'elles sont une seconde nature ». (Ibid., p.113).

Plusieurs écrits et rapports ont été édités concernant la prise en charge des Mineurs Non Accompagnés. Le guide Foire Aux Questions. Mineurs Non Accompagnés, proposé par le ministère de la justice en 2016, reprend le cadre législatif, les modalités de fonctionnement de la cellule nationale, la procédure d'évaluation, et l'orientation. La brochure réalisée par l'association FTDA ${ }^{11}$ en 2017, intitulée L'accueil et la prise en charge des Mineurs Isolés Étrangers en France, réaffirme les droits des usagers une fois dans le dispositif de l'Aide Sociale à l'Enfance et s'intéresse aux parcours d'insertion. Quant au dossier Mineurs non accompagnés: quels besoins et quelles réponses?, publié en février 2017 par l'ONPE ${ }^{12}$, il met en avant les besoins spécifique de ces jeunes et présente des dispositifs départementaux innovants. La CNAPE ${ }^{13}$ édite en février 2018 le rapport Accompagner et accueillir les Mineurs Non Accompagnés au regard de leurs besoins, qui donne lieu à des préconisations sur la prise en charge de ce public et sur l'homogénéisation des pratiques au niveau national. Enfin, I'UNHCR ${ }^{14}$ publie en décembre 2018 une étude, C'est bien qu'on nous écoute, donnant la parole aux Mineurs Non Accompagnés pour savoir comment ils vivent le parcours qui les mène de l'évaluation à l'entrée dans les services éducatifs.

Par ailleurs, nous avons retenu trois films documentaires particulièrement parlants pour notre sujet d'étude. Migrants - retour d'enfer, de Patrick Fandio (2017), recueille des témoignages de migrants revenus en Côte d'Ivoire après le dangereux voyage à destination de l'Eldorado européen, mettant en avant le caractère traumatique de l'expérience. A travers son film intitulé J'ai marché jusqu'à vous - récits d'une jeunesse exilée, (2016), Rachid Oujdi relate les obstacles rencontrés pour accéder à la mise à l'abri et à une prise en charge au sein des services de protection de l'enfance. Enfin, en 2018, le jeune réalisateur guinéen Thierno Souleymane Diallo

\footnotetext{
${ }^{11}$ France Terre D’Asile.

12 Observatoire National de la Protection de l'Enfance.

${ }^{13}$ Convention Nationale des Associations de Protection de l'Enfance.

${ }^{14}$ Haut-Commissariat des Nations Unies pour les Réfugiés.
} 
réalise Difficile à raconter (Nô Mëti Sîfâdhe), dans lequel, à travers les portraits de Mineurs Non Accompagnés installés en Gironde, il évoque les désillusions et il alerte sur la désinformation opérée dans les pays d'origine.

Les rapports et les documentaires donnant la parole aux jeunes sont ceux qui se rapprochent le plus de notre étude par la forme. Ils ciblent néanmoins une étape précise du parcours, alors que notre recherche englobe les trois périodes prémigratoire, migratoire et post-migratoire. Notre objectif n'est ni de décrire les phénomènes, ni d'évaluer les dispositifs, ni de dénoncer dans une posture militante. Appréhender les trajectoires de ce public sous l'angle sociologique des rôles sociaux constitue une approche nouvelle.

\section{DES INSTITUTIONS AUX RÉSEAUX INFORMELS}

Nous avons donc choisi d'aller à la rencontre des Mineurs Non Accompagnés pour recueillir leurs expériences et leurs subjectivités. L'entretien semi-directif nous est apparu approprié : laisser émerger la parole librement tout en orientant par des questions. Afin de faciliter les échanges, nous avons mis en place une méthodologie en deux temps. La première étape consistait en l'animation d'un entretien collectif (à trois ou quatre) à partir du visionnage du film documentaire J'ai marché jusqu'à vous - récits d'une jeunesse exilée, cité précédemment (Oujdi, 2016). Nous allions ensuite recevoir ces mêmes jeunes à l'occasion d'entretiens individuels. Les observations effectuées dans le cadre des pratiques professionnelles auprès de ce public viendraient bien entendu appuyer l'étude. Ayant dans l'idée que le parcours à l'Aide Sociale à l'Enfance avait un impact sur le vécu de la situation post-migratoire, nous avons opté pour une sélection de terrains de stage couvrant à la fois les différentes étapes de la prise en charge et des modes d'accueil diversifiés. Il s'agissait en effet de rencontrer des jeunes durant la phase d'évaluation, le temps de leur accueil à l'Aide Sociale à l'Enfance et une fois sortis du dispositif.

Il est à souligner un paramètre qui constitue une limite de l'étude, à savoir qu'en l'absence d'interprètes, les personnes interrogées devaient avoir une maîtrise minimum de la langue française. Une partie des jeunes a de fait été écartée de la démarche, principalement dans les services de premier accueil, et certaines nationalités ne sont pas représentées. Toutefois, la surreprésentation dans l'échantillon de jeunes en provenance d'Afrique subsaharienne est en adéquation avec les chiffres rencontrés dans les services. Notons également que selon les niveaux de langue, tous n'avaient pas les mêmes facilités à s'exprimer, ce qui nécessitait régulièrement de reformuler pour éviter tout malentendu. De plus, les accents, lorsqu'ils étaient difficiles à comprendre, ont complexifié les échanges et la retranscription des entretiens. 


\section{Terrain d'étude $\mathbf{n}^{\circ} \mathbf{1}:$ le SAEMNA}

En Gironde, la mission d'évaluation, qui depuis 2009 s'effectuait au DROMIE $^{15}$, rattaché au $\operatorname{CDEF}^{16}$, a été confiée en 2013 au SAEMNA ${ }^{17}$, géré par l'association le $\cos ^{18}$. Une semaine $d^{\prime}$ immersion nous a permis d'observer le fonctionnement de ce service aux missions si particulières et d'en comprendre les enjeux. Huit entretiens ont pu être menés auprès de jeunes pour qui la minorité avait été reconnue, accueillis à I'hôtel le temps que l'orientation soit effective vers une structure de l'Aide Sociale à l'Enfance. Écarter ceux qui se trouvaient en phase d'évaluation visait ainsi à éviter toute ambiguïté entre les missions du SAEMNA et notre étude. Alors que nous craignions qu'il ne soit compliqué d'aborder certaines questions délicates à cette étape décisive du parcours, les échanges qui se sont enclenchés sont parmi les plus riches, peut-être par habitude des entretiens à leur arrivée sur le territoire, ou du fait qu'un espace de parole décentré de l'évaluation venait constituer un nouveau souffle pour eux.

\section{Terrain d'étude $\mathbf{n}^{\circ} \mathbf{2}$ : le Squid}

Ce précédent stage a aussi révélé l'existence d'autres catégories de jeunes qu'il nous a semblé judicieux de rencontrer. Il s'agit de ceux qui ont été enregistrés au SAEMNA ${ }^{19}$ mais pour qui la mise à l'abri et l'évaluation n'ont pas encore débuté, et de ceux pour qui la minorité n'a pas été reconnue et qui ont déposé un recours en justice. $C^{\prime}$ est vers les services de prévention spécialisée que nous nous sommes alors dirigée. Leur action s'adressant aux jeunes en risque de marginalisation, ils sont effectivement amenés à rencontrer ces publics. C'est particulièrement le cas sur le secteur de Bordeaux centre. Nous avons ainsi été introduits au centre social autogéré dit «le Squid ", dans le squat dédié aux Mineurs Non Accompagnés. Une première rencontre nous a permis de présenter l'étude aux jeunes qui se sont montrés très curieux. II nous aura fallu toutefois défendre auprès de l'organisateur la démarche déconnectée de toute visée caritative, pour que la porte nous soit finalement ouverte. C'est ainsi qu'au cours de deux demi-journées, nous avons interrogé cinq jeunes portés volontaires, dont quatre en voie de recours et un inscrit au SAEMNA mais pas encore mis à l'abri. La passation des entretiens a requis une certaine souplesse dans la forme, la salle mise à disposition étant un lieu de passage. Dans la plupart des cas, la prise de notes a été privilégiée à l'enregistrement, du fait du contexte spécifique.

\footnotetext{
${ }^{15}$ Dispositif de Recueil et d'Observation des Mineurs Isolés Étrangers.

${ }^{16}$ Centre Départemental de l’Enfance et de la Famille.

${ }^{17}$ Service d'Accueil et d'Évaluation des Mineurs Non Accompagnés.

${ }^{18}$ Comité des CEuvres Sociales.

${ }^{19}$ Service d'Accueil et d'Évaluation des Mineurs Non Accompagnés.
} 


\section{Terrain d'étude $\mathbf{n}^{\circ} \mathbf{3}$ : le SRSA}

Pour ce qui est des jeunes pris en charge à l'Aide Sociale à l'Enfance, majoritaires dans l'échantillon, nous avons souhaité diversifier les types de structure et d'accueil. Les critères retenus étaient I'OPP ${ }^{20}$ ou l'APJM ${ }^{21}$, I'hébergement collectif ou individuel, l'accueil dans un service dédié à ce public ou en $\mathrm{MECS}^{22}$ classique, et enfin, l'admission directe ou dans le cadre de la péréquation. Les premiers entretiens ont été effectués au sein du SRSA ${ }^{23}$ de l'association laïque du Prado, une MECS classique accueillant des jeunes entre 16 et 21 ans, dont les Mineurs Non Accompagnés représentaient au moment de l'étude un tiers de l'effectif. La connaissance de la structure et la proximité avec l'équipe éducative ont favorisé la mobilisation des jeunes. II avait été convenu d'écarter ceux que nous connaissions pour éviter toute ambiguïté entre l'accompagnement éducatif et la recherche. Nous en avons ainsi rencontré huit, dont quatre avec lesquels nous avons expérimenté l'entretien collectif. Les échanges se sont rapidement enclenchés à partir du support vidéo, ce qui a favorisé une grande fluidité lors des entretiens individuels qui ont suivi. Cette formule collective, bien qu'efficace, n'a cependant pas pu être réitérée sur les autres terrains de stage pour des questions d'organisation et face à une difficulté, parfois, à mobiliser les jeunes.

\section{Terrain d'étude $n^{\circ} 4$ : le CAMINA}

Nous avons opté ensuite pour un stage au CAMINA ${ }^{24}$, ouvert par l'association laïque du Prado en janvier 2018 suite aux appels à projets du Conseil Départemental de la Gironde. Il nous intéressait de recueillir les ressentis des jeunes accueillis dans ce dispositif dédié spécifiquement à ce public. II propose des modes de prise en charge diversifiés afin de favoriser des parcours individualisés, dans différents territoires du département. Le stage devait avoir lieu à Langon, structure qui accueille les jeunes au sein de plusieurs colocations. Un seul a cependant accepté de participer à l'étude, les autres étant peu disponibles après leurs journées de formation et peu enclins à répondre de nouveau à des questions, sachant qu'ils avaient déjà été sollicités par la presse peu de temps auparavant. C'est finalement au sein d'une autre unité située à Bordeaux Caudéran et accueillant dans une "petite maison " cinq jeunes, avec présence quotidienne d'une équipe éducative sur place, que le stage s'est poursuivi. Sur les cinq, nous avons dû renoncer à mener l'entretien avec l'un d'entre eux qui allait trop mal pour pouvoir s'exprimer (il n'est pas comptabilisé dans l'échantillon). Un autre a accepté de jouer le jeu mais son mal-être a également constitué un frein à

\footnotetext{
${ }^{20}$ Ordonnance de Placement Provisoire.

${ }^{21}$ Accueil Provisoire Jeune Majeur.

${ }^{22}$ Maison d'Enfants à Caractère Social.

${ }^{23}$ Maison d'Enfants à Caractère Social.

${ }^{24}$ Carré d'Accueil pour les Mineurs Non Accompagnés.
} 
l'échange et les données sont peu exploitables (nous l'avons malgré tout inclus dans l'étude car ses réponses défensives traduisent quelque chose de sa subjectivité). Réaliser l'étude se révélait laborieux et peu confortable. Trois entretiens ont finalement pu être menés à bien. En complément, nous avons rencontré plus tard un autre jeune pris en charge au CAMINA de Lormont, dans le service de chambres en ville.

\section{Terrain d'étude $\mathbf{n}^{\circ} \mathbf{5}: \mathbf{R} \mathbf{d}^{\prime}$ accueil}

Nous avions donc interrogé des mineurs et des majeurs, hébergés en individuel, en colocation, ou dans des "petites maisons ", au sein de $\mathrm{MECS}^{25}$ aussi bien classiques que dédiées à ce public. Le dispositif $\mathrm{R}$ d'Accueil nous apparaissait alors comme incontournable pour recueillir le vécu des jeunes à la fois du collectif et de la péréquation. Créé en novembre 2016 suite au démantèlement de la jungle de Calais, il accueille désormais quarante Mineurs Non Accompagnés dans un foyer situé à Mérignac, dans le cadre de la répartition nationale et à titre provisoire. Précisons que ses missions et ses modes d'accueil évoluent et se diversifient avec le développement en mai 2019 d'hébergements en colocation. L'équipe a accueilli le projet d'étude avec enthousiasme, curieuse de découvrir ce public sous un autre angle et dans des contextes différents de celui de la péréquation et du collectif qui peuvent générer des tensions. II s'est cependant avéré compliqué de mettre en place les entretiens à une période où le service se restructurait. Par ailleurs, une maîtrise minimale de la langue française était un critère que tous ne remplissaient pas. Nous avons finalement interrogé quatre jeunes. Parmi eux, deux n'étaient arrivés que quelques jours auparavant et avaient trop peu de recul sur leurs situations. Les deux autres, plus anciens, étaient pris dans les changements institutionnels et parvenaient difficilement à se décaler des revendications apparemment collectives. Plutôt que de réserver ces entretiens à la semaine de stage dédiée, il aurait été plus judicieux de les répartir sur une période plus longue avec davantage de souplesse.

\section{Terrain d'étude $n^{\circ} 6$ : les jeunes sortis du dispositif}

Enfin, nous envisagions, pour compléter l'étude, d'interroger des jeunes qui, après une prise en charge à l'Aide Sociale à l'Enfance en tant que Mineurs Non Accompagnés, avaient quitté le dispositif. Nous souhaitions en effet recueillir leurs témoignages avec quelques années de recul, en vue de mesurer l'impact qu'avait pu avoir dans leurs parcours la rencontre avec les services. Nous avons rencontré à ce titre I'ADEPAPE $33^{26}$ qui reçoit effectivement trois Mineurs Non Accompagnés

\footnotetext{
${ }^{25}$ Maisons d’Enfants à Caractère Social.

${ }^{26}$ Association Départementale d'Entraide des Personnes Accueillies en Protection de l'Enfance.
} 
anciennement sous mesure éducative. II n'a cependant pas été possible de nous mettre en lien avec eux pour des raisons éthiques. Nous avons aussi interpellé les inspecteurs du Conseil Départemental mais il a été convenu qu'il serait plus pertinent de passer directement par les services éducatifs. Les équipes que nous avons sollicitées n'étaient toutefois pas en mesure de nous transmettre ces contacts. C'est finalement par le biais du SRSA ${ }^{27}$ que nous avons pu rencontrer deux jeunes. Pour ne pas biaiser les réponses, nous souhaitions écarter ceux que nous avions accompagnés. Nous avons malgré tout fait un écart pour l'un d'entre eux avec qui le contact était maintenu et pour qui la fin de prise en charge avait libéré la parole. II avait manifesté la possibilité et le désir de « raconter » une fois qu'il serait sorti.

Mener cette étude a donc nécessité de pousser la porte des institutions et c'est grâce au soutien de différents responsables associatifs que nous avons pu avoir nos entrées. Au sein de chaque service, les échanges avec les professionnels sont venus alimenter les réflexions. En parallèle aux stages, un entretien avec la présidente et le vice-président de I'ADEPAPE $33^{28}$ a ouvert un autre regard sur les parcours à l'Aide Sociale à l'Enfance, celui d'anciens enfants placés. Une rencontre avec une psychologue de la consultation transculturelle de l'association Mana a également été très riche d'enseignements sur les questions de reconstruction après les traumatismes liés aux parcours migratoires, mais aussi de filiation et de passage à l'âge adulte. Nous ferons référence à ces apports dans le mémoire.

\section{DES RÉCITS, DES TENDANCES}

Sur les trente-trois entretiens menés, vingt-trois ont été enregistrés et la prise de notes a été privilégiée pour les dix autres, soit par réticence des jeunes face au dictaphone, soit parce que le contexte y était peu favorable. Nous avons testé la retranscription intégrale pour l'enregistrement de l'entretien collectif, mais cette méthode, bien que précise techniquement, inhibait la réflexion et le début d'analyse du contenu. Nous avons donc opté par la suite pour une retranscription synthétique avec collecte de verbatim, essentiels pour faire ressortir la subjectivité des acteurs. Précisons que les prénoms des personnes ont été changés dans l'écrit par mesure de discrétion.

Puisqu'il s'agissait non pas d'une étude quantitative mais qualitative, l'analyse ne visait nullement l'établissement de statistiques mais bien la mise en évidence de tendances dans les logiques d'action des jeunes interrogés et dans leurs ressentis face aux situations vécues. En s'attachant à la conception de Neal Gross du « rôle social »

\footnotetext{
${ }^{27}$ Service de Réadaptation Sociale pour Adolescents.

${ }^{28}$ Association Départementale d'Entraide des Personnes Accueillies en Protection de l'Enfance.
} 
entendu comme "les réactions d'un acteur aux attentes de son environnement " (Coenen-Huther, 2005), nous allions prendre en considération les deux environnements différents que sont d'une part le pays d'origine et la famille, d'autre part la société d'accueil et ses institutions.

La première partie du mémoire portera ainsi sur l'étude du contexte prémigratoire, en lien avec la culture migratoire, mais aussi en référence aux situations familiales dans le pays d'origine et à la manière dont s'organisent les départs. Cet aperçu donnera des indications sur les motifs, de même que sur la part de décision revenant aux jeunes, sur leurs rôles vis-à-vis des familles et sur leurs attentes à l'égard de l'Europe. Nous pourrons mettre en lien les modes migratoires repérés avec la typologie d'Angélina Etiemble et Omar Zanna (2012). Pour comprendre le sens profond de ces mobilités, nous nous appuierons sur des auteurs comme Thomas Fouquet et Francesco Vacchiano qui les attribuent à une quête de statut. Le critère de l'origine géographique sera à prendre en compte en tant qu'il est susceptible d'influer sur les résultats, même si nous ne prétendons pas pouvoir généraliser les éléments recueillis, certaines nationalités étant peu représentées dans l'échantillon sélectionné. De plus, les données relatives aux contextes de départ seront à manier avec prudence au regard des difficultés, voire de l'impossibilité pour certains jeunes à dire leur histoire, soit de par son caractère douloureux, soit par stratégie pour accéder à la prise en charge à l'Aide Sociale à l'Enfance. C'est selon nous un biais dont nous ne pouvons faire l'économie lorsque nous nous intéressons à ce public et qui nous a longuement questionnée au cours de l'étude, venant parfois mettre en doute sa pertinence. Les motifs de départ ne constituent néanmoins qu'une question parmi d'autres qu'il s'est agi de dépasser pour nous intéresser aux vécus de ces trajectoires migratoires et postmigratoires.

La seconde partie mettra en avant le rapport des jeunes à leur environnement actuel et aux institutions françaises. II s'agira, à chaque étape de la prise en charge à l'Aide Sociale à l'Enfance, de repérer les niveaux d'information et de compréhension qu'ont les jeunes des exigences qui leur sont posées, mais aussi leurs priorités et éventuellement leurs inquiétudes. Le passage à la majorité sera un critère essentiel à prendre en compte en ce qu'il induit de nouveaux enjeux. Le concept de "pensée d'État » développé par Abdelmalek Sayad éclairera notre réflexion.

Finalement, croiser les rôles sociaux dans le pays d'origine et dans la société d'accueil place les jeunes au cœur des dialectiques là-bas/ici, passé/présent. Cela revient à interroger les rôles sur l'échelle spatio-temporelle. C'est sous le prisme de la sociologie de la jeunesse que nous étudierons dans une troisième partie le public des Mineurs Non Accompagnés, avec Pierre Bourdieu et Olivier Galland comme principales 
références. Nous observerons ainsi comment s'effectue, pour ces jeunes, le passage à l'âge adulte. Derrière le critère administratif de l'âge que pose et impose notre société, c'est la dimension culturelle qui sera mise au premier plan.

\section{QUELQUES HISTOIRES DE VIE INTRODUCTIVES}

Ouma a 17 ans et est guinéen. II n'a pas connu ses parents et a été élevé par sa grand-mère avec son frère jumeau. Ce dernier est décédé très jeune, des voisins lui ayant jeté un mauvais sort dans le village. A l'âge de 7 ans, sa grand-mère ne pouvant pas financer sa scolarité, Ouma a été adopté par un ami prénommé Mahmoud. II a alors vécu avec "sa nouvelle famille " et allait à l'école. Mahmoud s'est séparé et remarié. Lorsque la seconde épouse a appris qu'Ouma n'était pas un fils biologique, elle l'a traité différemment. II n'allait plus à l'école tous les jours car il devait aider aux tâches quotidiennes de la maison. Mahmoud est tombé malade et a dû interrompre son travail, si bien que c'est l'épouse qui gérait le foyer. Un jour, le fils aîné de Mahmoud a volé l'argent de sa " tante " et a proposé à Ouma de partir. Il avait alors 15 ans. Il a beaucoup hésité car il ne voulait pas le faire sans informer son père adoptif. Ils ont finalement pris la route vers le Mali. Ouma explique avoir connu la guerre et les campements de clandestins. Il avait très peur et voulait rentrer mais ne pouvait plus faire marche arrière. Ils ont passé dix-huit mois dans la forêt au Maroc, puis Ouma a perdu la trace de son "cousin". II a finalement traversé la Méditerranée vers l'Espagne et a séjourné dans un centre pour mineurs à Motril, mais ne parlant pas la langue, il a préféré poursuivre vers la France. Lorsque nous le rencontrons, cela fait quatre mois qu'il est au SAEMNA ${ }^{29}$. Il attend une place dans un foyer.

Farhad, de nationalité afghane, a 17 ans. Il est arrivé en France à l'âge de 15 ans. Il a deux sœurs aînées, une plus jeune et un petit frère. Lorsqu'ils étaient enfants, ils vivaient dans un village, mais les trajets jusqu'à l'école étaient longs et la route parfois coupée du fait de la montée des eaux. La famille a finalement déménagé à Kaboul afin de faciliter la poursuite d'études. Lorsqu'il avait 13 ou 14 ans, Farhad a décidé de partir pour l'Europe. II a dû s'y reprendre à plusieurs reprises, car les frontières étaient fermées et il s'est fait arrêter. Ses parents soutenaient son projet, lui souhaitant de pouvoir suivre ses études en sécurité. Trouver du travail en Afghanistan était par ailleurs compliqué sans réseau. La traversée a été très difficile et elle lui a pris huit mois. Aujourd'hui, Farhad se sent bien. Il passe un CAP $^{30}$ électricien, en apprentissage. Il est pris en charge par le SRSA ${ }^{31}$ et est hébergé en famille logeuse. Une de ses sœurs

\footnotetext{
${ }^{29}$ Service d'Accueil et d'Evaluation des Mineurs Non Accompagnés.

${ }^{30}$ Certificat d'Études Professionnelles.

${ }^{31}$ Service de Réadaptation Sociale pour Adolescents.
} 
aînées est arrivée en France avec son mari il y a quelques mois et il leur rend visite régulièrement. II est également en contact avec ses parents restés au pays et il aimerait plus tard les faire venir. II explique que c'est souvent de cette façon que l'on procède chez lui, à savoir qu'une personne part en premier et facilite ensuite le regroupement familial.

Semei avait 14 ans lorsqu'il a décidé de quitter la Côte d'Ivoire. II voulait en effet rejoindre l'oncle d'un ami installé en Libye car il avait entendu que s'il parvenait à se faire sa place dans le football, il pourrait gagner de l'argent. Ses parents lui ont demandé de passer d'abord son brevet. Sa mère le trouvait trop jeune pour partir. Son père, quant à lui, trouvait bénéfique qu'il puisse travailler à l'extérieur. En tant que garçon, il était préparé à quitter un jour la maison. Depuis l'âge de 8 ans, Semei se levait tous les jours à 3 h00 du matin pour aider sa mère, commerçante. Le voyage s'est préparé et il a finalement pris la route pour la Libye. Là-bas, il a été incarcéré durant trois mois, puis acheté. II a alors fait un an de travail forcé pour une personne qui l'a ensuite embauché, avec salaire. Semei a séjourné sept mois de plus chez elle, avant de s'enfuir. II est passé par l'Italie et a rejoint la France en août 2017. De Cannes à Paris, puis à Nevers, où il a été évalué, sans être reconnu mineur. Face au froid de Paris et à l'absence de place dans les foyers, il a finalement choisi Bordeaux comme destination, pour l'équipe de football des Girondins. Il est arrivé en avril 2018. Étant donné qu'il avait déjà été évalué, il a dû entamer un recours en justice, période durant laquelle il a vécu dans un squat. II a alors été mis en relation avec différentes associations. Son premier accueil en foyer s'est mal passé. Avec les autres jeunes, ils ont dénoncé les conditions de prise en charge. Semei a pu faire reconnaître ses droits en s'appuyant sur son réseau associatif et a finalement été orienté au CAMINA ${ }^{32}$. II a aujourd'hui 18 ans et suit une formation en boulangerie. Il attend la délivrance de son premier titre de séjour.

Oussy est Malien et il est majeur depuis quelques mois. II explique avoir quitté son pays sans informer sa famille. Un matin, il a pris son sac à dos et il est parti avec un ami. Son père lui avait fait arrêter l'école pour travailler aux champs, alors qu'Oussy souhaitait poursuivre ses études. Comme il savait qu'il ne réussirait pas à le convaincre, il a choisi de prendre la route. Oussy voyait souvent des gens partir travailler à l'extérieur et revenir en hiver. Cela éveillait sa curiosité. II pensait lui aussi ne s'absenter que quelques mois. Il a d'abord travaillé dans un autre village et lorsque son ami est rentré, lui a décidé de suivre d'autres personnes qui partaient pour l'Algérie. De là, la Libye où il a subi l'exploitation, l'Italie, puis la France. Ce n'est

\footnotetext{
${ }^{32}$ Carré d'Accueil pour les Mineurs Non Accompagnés.
} 
qu'arrivé ici qu'il a recontacté sa mère, mais son absence avait suffi à lui faire comprendre qu'il avait pris le chemin de la migration. Après avoir passé trois mois à Cahors, dans un foyer, puis dans une famille d'accueil, il a été « envoyé " à Bordeaux. II ne savait pas qu'il y avait des dispositifs spécifiques pour mineurs en Europe et n'en comprenait pas les fonctionnements au début. Il était perdu. Aujourd'hui, il est en formation d'électricien et passe son $\mathrm{CAP}^{33}$. II compte ensuite poursuivre sur un baccalauréat. Oussys vit en colocation avec d'autres jeunes du service. Plus tard, il ne sait pas encore s'il restera en France ou s'il repartira. Aider sa famille, il n'y pense pas forcément. Peut-être sa mère, mais son père non, il est trop dur et de toute façon, il n'en a pas besoin. L'aider, ce serait travailler avec lui dans les champs. Oussy n'est pas parti comme d'autres pour soutenir sa famille financièrement mais pour les études.

${ }^{33}$ Certificat d'Aptitude Professionnelle. 


\section{PARTIE 1. UNE MIGRATION AUX CONTOURS PARTICULIERS}

\section{UNE EXPÉRIENCE NOUVELLE DE LA MIGRATION}

\subsection{Culture de la migration et imaginaire migratoire}

Dans certaines régions ou villes du monde, les départs sont fréquents. Les inégalités territoriales et la mondialisation favorisent la migration. On grandit au contact de ces mobilités. On voit un cousin ou un voisin partir, puis revenir à l'occasion de visites ponctuelles. Ces vies dont on se fait le spectateur nourrissent l'imaginaire. Dans certains cas, même, on sait que les enfants prendront la route.

"Ma famille savait que j'allais partir. Maintenant, dans mon pays, il n'y a presque que des vieux, toute la jeunesse est partie ! Les écoles ont fermé car il n'y a plus d'élèves. II y a trop de corruption. Je ne pense pas que mes parents étaient d'accord mais quand tu vois tous les gens qui partent, tu as envie toi aussi, même si tu ne sais pas où tu vas. " Anton, kosovar, 25 ans.

"Nous, notre communauté migre beaucoup. C'est culturel. Nous sommes des éleveurs et des commerçants. C'est comme ça. C'est pour cela qu'on dit que les Peuls en Afrique $n^{\prime}$ ont pas d'origine. A chaque fois, on se déplace d'un point $A$ à un point $B$. Et puis dans ma ville, il y avait tellement de jeunes qui étaient sortis! Certains volaient les parents pour partir!»

Ange, guinéen, 17 ans.

C'est Jeannette Schoorl qui propose en 2000 l'expression «culture de la migration " pour désigner "les modifications qu'induit au sein des sociétés d'origine l'expérience vécue au loin par les migrants » (dans Fouquet, 2007). II ne s'agit pas uniquement de voir partir. On est impacté par ce que vivent les migrants. Le premier élément qui frappe les yeux, c'est leur confort matériel. A leur tenue vestimentaire, on sait qu'ils ne manquent de rien. Nous ne serons pas surpris alors d'observer des Mineurs Non Accompagnés s'attacher à l'apparence physique. Comme tout adolescent ? Sûrement, mais avec une dimension en plus, celle de la supposée réussite sociale.

Au contact de ceux qui sont partis, donc, on rêve d'un ailleurs, d'une autre vie. «L'ailleurs dont il est ici question exprime un espace d'imaginaires dépositaire des aspirations à un mieux-être et à un mieux vivre. [...] Mais il demeure néanmoins largement du domaine de l'imaginaire, car il se construit surtout sur la base de représentations et fantasmes élaborés sur ces sociétés, qui n'ont pas ou peu d'équivalents dans le tangible. " (Fouquet, 2007). Sur les trente-trois jeunes interrogés, vingt et un avaient une idée de la France avant de partir et ces représentations étaient 
positives. Ils évoquent la possibilité d'une vie meilleure, les facilités pour réaliser les projets, le développement des infrastructures, la gratuité de l'école et des soins, le niveau élevé des diplômes, les opportunités de réussite dans le football ou encore une histoire commune et un supposé bon accueil aux étrangers. Ceux qui disent ne pas avoir eu d'informations n'ont nullement choisi la migration ou ne visaient pas forcément la France, souhaitant rejoindre l'Europe ou décidés à partir "n'importe où ».

" J'entendais que la France était un beau pays et que là-bas, tout était facile. Là-bas, tu peux travailler facilement. Tu peux gagner de l'argent facilement. Là-bas, tu peux vivre ta vie, tu n'as pas de problèmes. "

François, camerounais, 18 ans.

"Tu te dis que tu vas aller en France, en Europe. Ici, en Europe, dès que nous, les Noirs on arrive ici, on croit qu'on est entré au paradis. On se dit directement qu'on a trouvé Dieu quoi ! On est venu voir le Dieu. Tu vois? C'est un truc que je vous dis, franchement, au fond de mon cœur. Les Français sont Dieu. Les Blancs sont Dieu devant nous. " Issan, guinéen, 18 ans.

Ce n'est qu'une fois sur place que l'on peut confronter l'imaginaire à la réalité, rencontre qui peut être source de désillusion. Comme l'énonce Joël Fronteau, «les désillusions proviennent en grande part de l'écart entre le pays rêvé et la situation réelle " (dans Legault, 2000, p.28). Certains jeunes expliquent avoir reçu des informations erronées ou partielles. On leur avait parlé des difficultés de la traversée mais pas des obstacles rencontrés sur place. Ils ne trouvent pas ce à quoi ils s'attendaient.

"Je vous ai dit qu'en Afrique, on se met dans la tête que la France, c'est le paradis. Chez moi, c'est comme ça. La France, c'est le paradis de chez nous. Donc je ne regrette pas. $C^{\prime}$ est juste que je ne m'attendais pas à des trucs comme ça ! "

Issan, guinéen, 18 ans.

De fait, "l'exil imaginaire" dont parle Thomas Fouquet en tant que désinvestissement des modes de vie des aînés en parallèle à un surinvestissement des images de l'Ailleurs fantasmé, ne laisse pas la place au doute ni à la nuance. L'ailleurs est forcément bon et signe de réussite sociale. Ceux qui reviennent taisent les difficultés et même s'ils racontaient, on ne les croirait pas. On s'attache à l'espoir d'une vie meilleure, on met tout mettre en œuvre pour y accéder et on ne recule pas. Thomas Fouquet décrit ce phénomène: "Au demeurant, celui qui revient porteur d'avertissements et de mises en garde n'est souvent pas audible : on le suspecte de vouloir dissuader les autres de partir à leur tour, afin de garder pour lui seul tout le prestige de l'Ailleurs. À cela s'ajoute l'ironie parfois désabusée de ceux qui ont vu les deux côtés du miroir, et qui veulent en tirer les bénéfices au moins symboliques : les 
efforts et souffrances consentis loin du pays sont rendus acceptables par la seule certitude que l'on reviendra en champion, par la conscience que l'on a d'être devenu, à travers la migration, une potentielle " nouvelle figure de la réussite " (Banegas et Warner, 2001). (Dans Fouquet, 2007). Si l'on rentre au pays, on a forcément réussi. L'échec serait source de déshonneur. Comme en témoigne l'inquiétude de Redei au moment de faire sa valise pour le Cameroun. Il est pourtant à quatre mois du départ. Préparer sa valise, c'est mettre de l'argent de côté pour se racheter des vêtements neufs et faire le plein en cadeaux. II ne parvient pourtant pas à joindre les deux bouts en fin de mois.

"Tu demanderas à un Camerounais, ce que signifie repartir chez lui! Tu imagines ce qu'ils vont dire si je ne suis pas bien habillé et si je n'ai pas les cadeaux ? Ils vont dire que je souffre en Europe... Et que je n'ai pas réussi ma vie !»

Redei, camerounais, 21 ans.

Le "mensonge collectif », tels sont les termes utilisés par Abdelmalek Sayad pour nommer ce phénomène qui consiste à idéaliser son parcours en niant les difficultés, offrant ainsi une vision idyllique et justificatrice de son exil. Un « mensonge » qui explique en partie la perpétuation de l'émigration. (Témime, 1999)

"Ceux qui sont arrivés avant toi, lorsque tu es encore derrière, ils te disent des choses et ce n'est pas ce que tu vas trouver ici. Quand tu arrives, tu vois que ce n'est pas... En fait ce qu'ils t'ont dit, c'était en grande partie de fausses idées. Ils savent que s'ils te disent que c'est difficile, compliqué, ça va te décourager et tu n'auras plus le courage d'affronter ce qu'il y a dehors. Quand tu as tellement souffert, tu ne veux plus rien comprendre. Ton objectif, c'est d'avancer, plus de retourner. [...] Si je conseille de ne pas prendre la route et de ne pas venir, on va me répondre que si la route était si mauvaise, je ne serais pas ici. Les gens pensent que si j'ai réussi, ils le peuvent aussi. Après, tu es obligé de les encourager. Tu ne peux pas bloquer les frontières. "

François, camerounais, 18 ans

Thomas Fouquet (2007) ajoute que " les discours qui tendent à déconstruire les illusions sur l'Ailleurs sont encore moins acceptables lorsqu'ils sont le fait d'un " autresemblable ", dans la mesure où, pour reprendre les termes de Jodelet, celui-ci est le "médiateur de l'identité, de la représentation et de la connaissance en ce qu'il complète, réfléchit et donne sens à ce que le sujet éprouve. " Les " aventuriers ", pour reprendre la dénomination utilisée par François, s'identifient à "ceux qui sont devant ». Ils sont passés par là et ont surmonté les difficultés. Croire en leur succès revient à croire en sa propre réussite. Constater leur échec serait source de découragement. 
Paradoxalement, une fois de l'autre côté, dans cet ailleurs fantasmé, perpétuer le "mensonge " peut enfermer dans un mal-être solitaire. La tenue vestimentaire, cette carapace qui préserve le mythe et que l'on attribuait au succès, semble aux yeux de celui qui souffre trompeuse. II ne peut dire ses difficultés, on ne le croirait pas. II est bien habillé. II ne manque de rien.

"Les gens, ils te jugent selon la façon dont tu es habillé mais ils ne savent pas ce que l'on endure ici, comment on vit ici. [...] Quand ils te voient bien habillé, dans leur tête, ils savent que tu vis bien. Et pourtant, ils ne se posent pas la question de savoir comment tu as fait pour arriver, ce que tu as vécu en route... "

François, camerounais, 18 ans.

Croire, rêver, se projeter, partir. Confronter l'imaginaire à la réalité. Vivre parfois une certaine désillusion. Se voir contraint de la taire. Cela fait partie du processus migratoire.

\subsection{Des contextes de départ diversifiés}

Les trente-trois jeunes rencontrés sont de différentes nationalités, même si une grande majorité est originaire de l'Afrique subsaharienne: treize Guinéens, six Maliens, cinq Ivoiriens, deux Camerounais, un Sénégalais, une Congolaise, un Gambien, un Algérien, deux Afghans et un Kosovar. Les circonstances de départ peuvent être analysées à partir de nombreux critères : sexe, place dans la fratrie, lieu d'habitation, niveau d'études, contexte politique, catégorie socio-économique... Faire ressortir des données chiffrées nécessiterait cependant de mener une étude quantitative auprès d'un échantillon plus large et ce n'est pas l'objectif puisque nous avons choisi de nous centrer sur la subjectivité des acteurs. Malgré tout, pour comprendre les vécus actuels de ces jeunes, il est inévitable de prendre en considération les contextes pré-migratoires. "L'expérience migratoire est à la fois un projet (de vie), un trajet (le voyage), un parcours (des étapes) [...]. La migration est d'abord une émigration, ensuite une immigration. Le processus migratoire ne saurait être envisagé sans ses prémisses : l'existence ou non d'un projet migratoire. Un tel projet permet de faire la distinction entre immigrants et réfugiés, entre d'une part des personnes qui envisagent, élaborent et réalisent un projet migratoire, et d'autre part des personnes qui doivent quitter leur pays contre leur gré. " (Fronteau dans Legault, 2000, pp.1-2)

La typologie proposée par Angélina Etiemble et Omar Zanna en 2012 constitue un bon point de départ pour étudier les différentes formes de migrations des Mineurs Non Accompagnés : mineurs mandatés, exilés, exploités, errants, fugueurs, aspirants et rejoignants. Ces qualificatifs traduisent en effet à eux seuls quelque chose du 
contexte de départ ou des objectifs visés. Ils sont plus précis que la théorie économique classique de la migration fondée sur les facteurs d'attraction "pull » (attirant les migrants vers leurs destinations), indissociables des facteurs de répulsion "push» (provoquant le départ de personnes hors de leurs pays d'origine). En effet, au-delà de ces motifs structurels, la classification d'Angélina Etiemble et Omar Zanna prend en compte des dimensions plus subjectives comme le rôle au sein de la famille, les modes de vie dans le pays d'origine, ou encore les aspirations individuelles. Cependant, faire cadrer chaque situation spécifique à une de ces catégories n'est pas évident car elles s'entremêlent.

Nous n'avons pas rencontré de "mineurs-errants", vivant déjà dans la rue au pays et ayant fait de cette errance un mode de vie. Constat qui peut s'expliquer du fait que ces jeunes ne sont généralement pas demandeurs de prise en charge. C'est le cas de jeunes Marocains qui se trouvent actuellement dans cette configuration et qui une fois aux portes du Conseil Départemental, repartent avant d'être accueillis dans les foyers dont les fonctionnements ne répondent pas à leurs attentes.

Les "mineurs-exploités" au sens d'une exploitation organisée dès le départ (prostitution, vol, travail clandestin...) sont également absents de l'échantillon, même si certains des jeunes rencontrés ont pu vivre ce phénomène dans le pays d'origine (c'est l'exemple de Sekou qui avait été confié à un " maître " au Mali) ou durant la traversée (comme Semei qui a subi le travail forcé en Libye). Les filles, majoritairement concernées, sont peu visibles de par leur vulnérabilité et échappent aux services de protection.

La catégorie du " mineur-rejoignant » concerne une des personnes interrogées, confiée par sa famille à un proche installé en France, avant que des difficultés relationnelles ne l'amènent à solliciter une mesure de protection.

Par ailleurs, trois jeunes entrent dans la catégorie des " mineurs-exilés ", fuyant pour deux d'entre eux un contexte de guerre ou d'insécurité (un Kosovar et un Ivoirien) et pour la troisième un mariage forcé.

Les autres "profils" présentent quant à eux des frontières plus floues. Pour exemple, le projet d'étudier, fréquemment cité comme motif de départ, peut tout aussi bien relever de l'aspiration que du mandat : aspiration à « s'émanciper du milieu familial et de la société d'origine ", " dans une forme de quête plus personnelle ", ou mandat familial consistant à « soutenir financièrement ou à étudier en vue d'acquérir 
un prestige social » (Etiemble et Zanna, 2013). Pour distinguer le " mineur-aspirant » du "mineur-mandaté ", nous pouvons introduire les paramètres du libre choix, de l'accord parental et de la responsabilité énoncée vis-à-vis de la famille. Critères à manier malgré tout avec prudence et souplesse. En effet, un jeune s'étant vu imposer le départ peut s'être rapidement approprié le projet et devenir aspirant. A l'inverse, le fait d'avoir initié la migration ou d'affirmer un choix de vie contre une décision parentale n'écarte en rien la possibilité de soutenir sa famille. II peut y avoir un mandat implicite. Ces paramètres servent donc de grille de lecture mais doivent être croisés pour être efficaces.

Sept jeunes cadrent avec la définition du " mineur-aspirant ", ayant pris seuls la décision de partir, pour accéder à une vie meilleure, réaliser un rêve ou étudier. Ils n'ont pas forcément de responsabilité à l'égard des familles. "Dans certains cas, le jeune est lui-même très déterminé à partir, y voyant la perspective d'un accomplissement personnel, tandis que sa famille peut être plus réticente à un tel projet. » (Bricaud, 2012, p.21).

Sept jeunes ont pour projet d'étudier, soit pour répondre aux attentes de prestige des parents, soit en vue plus tard d'aider leurs familles. Ce sont des « mineursmandatés-étudiants ». Même si le mandat n'est pas verbalisé, ils se doivent de soutenir leurs proches. II y a donc mandat implicite. Trois autres ont pour seul objectif de travailler et de soutenir financièrement leurs familles. Ce sont les "mineursmandatés-travailleurs ». " Le départ a souvent beaucoup à voir avec les décisions des adultes qui entourent les jeunes. Les familles donnent le mandat, explicite ou non, de se rendre dans un pays étranger pour y " réussir ", faire des études, travailler pour rembourser leur passage et éventuellement envoyer de l'argent à leurs proches restés au pays. L'émigration est alors envisagée comme une stratégie familiale de réponse à la pauvreté ou à l'insécurité du pays d'origine. " (Ibid., p.20).

En outre, neuf jeunes décrivent des circonstances et une configuration familiale dans lesquelles ils ne trouvaient pas leur place. Ils avaient perdu leurs parents ou en étaient séparés et vivaient auprès de la famille élargie qui n'avait pas forcément la possibilité de les prendre en charge. II est question aussi de relations conflictuelles avec les marâtres. Dans ces contextes inconfortables, ils ont pris la décision de fuir ou ont été emmenés pour plus d'opportunités ailleurs. Ils n'entrent dans aucune des catégories de la typologie d'Angelina Etiemble et Omar Zanna. Nous pouvons en ajouter une nouvelle que nous nommerons les « mineurs-éloignés ». Éloignés de leurs 
familles, éloignés par les personnes les ayant recueillis, à la recherche d'une place et d'un statut à travers la migration.

Pour résumer, dix jeunes se rapprochent des "mineurs-mandatés ", sept correspondent aux "mineurs-aspirants", trois sont des "mineurs-exilés", une peut être considérée comme une " mineure-rejoignante » et neuf fondent la catégorie des " mineurs-éloignés ». Pour les trois derniers qui n’ont pas été cités, nous n'avons pas d'éléments précis sur les circonstances de départ et leurs motivations.

Plusieurs éléments ressortent de cette analyse: une proportion élevée de mineurs aspirants, l'émergence de la catégorie des éloignés, la notion de mandat implicite. Aborder la question migratoire renvoie généralement à deux modèles, à savoir l'exil politique et la migration économique. La loi Asile et Immigration du 10 septembre 2018 en témoigne puisqu'elle renforce la distinction entre ces deux types de migrations. Chez les mineurs, la migration économique se traduit par le mandat. Partir travailler ailleurs pour soutenir financièrement ses proches. Mais aussi rembourser la dette contractée. On pourrait donc s'attendre à trouver parmi les jeunes rencontrés une majorité de mineurs-mandatés-travailleurs et exilés. Ce n'est pourtant pas le cas. Le mandat officiel s'estomperait donc au profit d'un mandat plus implicite. II n'est pas énoncé mais il est intégré, comme faisant partie de la culture familiale. Celui qui a, même peu, donne. "Quand tu as un peu ici, cela devient beaucoup là-bas » explique Issam. Pour en revenir au profil aspirant, ajouté en 2012 par Angélina Etiemble et Omar Zanna à la première classification, il se situe à l'antipode du mandaté. II renvoie à cette figure moderne du migrant que nous développerons en suivant. Les mineurs éloignés ne relèvent-ils pas eux aussi de cette nouvelle forme de migration, visant à se réaliser dans un ailleurs?

\subsection{La migration comme traduction d'un rapport moderne au monde}

"Le fait migratoire ne peut être considéré du seul point de vue de la société d'accueil. La démarche doit être inversée : logiquement et chronologiquement, il faut partir, comme l'immigré, du pays d'origine, pour comprendre les causes et le sens de l'immigration. " (Sayad dans Témime, 1999). Comprendre le sens de la migration, tel est l'objectif des différentes typologies dressées. Elles peuvent aussi permettre d'attirer l'attention sur certaines fragilités à prendre en compte dans les accompagnements. Pourtant, comme nous l'avons vu précédemment, cette catégorisation est compliquée à établir et reste incertaine. D'une part du fait que les frontières sont poreuses entre chaque type de migration, avec des causalités 
s'entrecroisant, d'autre part car les éléments recueillis sont parfois insuffisamment détaillés ou partiellement fiables.

Effectivement, le contexte de nécessité dans lequel se trouvent les Mineurs Non Accompagnés les conduit à développer des stratégies pour accéder à un statut en Europe et parfois à se reconstruire une histoire, ou du moins à " nuancer » la leur. Ils ne peuvent pas tout dire. L'authenticité des récits peut être mise en doute. " II est bien évident qu'arrivés ici, cette parole-là ne va pas être donnée aux enquêteurs, au personnel ni aux travailleurs sociaux, si ce n'est après la mise en œuvre d'un long chemin d'apprivoisement, [...] d'une mise en confiance installée dans une durée. [...] II y a donc construction du discours et mise en mot de l'histoire, en fonction des intérêts recherchés.» (Tanon, 2011). (Nous reviendrons sur cet aspect dans la dernière partie du mémoire). Les professionnels intervenant avec ce public le savent et font avec cette donnée. Il n'est question ni de porter un jugement ni de chercher à tout prix à connaître la vérité. L'essentiel est d'accompagner les jeunes là où ils en sont, à partir de ce qu'ils donnent à voir et laissent transparaître d'eux-mêmes. "Au lieu de mettre leur parcours et leur parole en doute, pensons plutôt que cela fait partie du processus. » (Ibid.)

Cependant, pour une étude sociologique, l'enjeu est autre. Comment tirer des conclusions à partir d'éléments incertains ? Par exemple, l'un des jeunes rencontrés, en deux temps, a évoqué successivement des contextes de départ différents, le faisant passer de la catégorie de " mineur-aspirant » à celle de " mineur-mandaté ». Dans les services d'évaluation de la minorité, les récits de vie sont recueillis en nombre et lorsqu'ils se répètent, à une période donnée, pour une même nationalité, il est à supposer qu'ils ont été appris, dictés par les passeurs. Certains jeunes déplorent d'ailleurs d'avoir reçu de mauvaises informations de leur part, mais aussi de compatriotes, durant la traversée. Comme Osei qui explique s'être débarrassé de ses documents d'identité et avoir déclaré qu'il ne vivait plus avec ses parents, ce qui n'était pas le cas, sur les conseils du passeur, pour accéder plus facilement à la prise en charge, ce qui l’a ensuite pénalisé.

Plutôt que de nous attarder sur les motivations au départ, nous allons donc nous en décaler pour nous recentrer sur le vécu des jeunes. Qu'il faille apporter quelques " nuances » aux récits de vie, que la configuration familiale décrite ne soit pas tout à fait la réalité, cela ne modifie en rien l'expérience de la situation post-migratoire. Les mots pour exprimer la subjectivité des acteurs sont authentiques. "La migration est une aventure mystérieuse et initiatique ; mystérieuse parce que, si l'on en comprend 
le déroulement, il n'en va pas de même pour ses motifs, et initiatique parce que c'est précisément dans son déroulement que l'on peut en discerner les motifs. " (Fronteau dans Legault, 2000). C'est donc sous l'angle de l'anthropologie de l'expérience que nous abordons le phénomène de la migration des Mineurs Non Accompagnés, entendue par Victor Turner et Edward Bruner (1986) comme étant « au croisement de données objectives (des faits concrets auxquels les individus se confrontent) et de données subjectives (les modalités de penser et d'agir face à ces faits) " (ClavéMercier, 2014, p.19). François Dubet, qui a consacré une partie de ses écrits à la notion d'expérience, la relie à la subjectivité des acteurs. "Ce qu'ils disent constitue leurs constructions de la réalité. " (1991, p.16). Il souligne les deux dimensions de l'expérience, à savoir que d'une part, "l'acteur est engagé dans une activité sociale ", d'autre part « il juge cette activité » (Ibid., p.29). II s'agit de mettre à distance le vécu et d'engager un travail de réflexivité.

Derrière toutes ces formes de migration se dissimule la nécessité ou l'envie d'un ailleurs: quitter un contexte (facteurs «push») ou accéder à un autre (facteurs "pull»). Fuir la misère économique (pour soi et/ou ses proches), échapper à un mariage forcé, s'extraire d'un milieu familial conflictuel ou encore préférer les études au travail exigé par la famille, reviennent à s'émanciper d'une voie toute tracée. Les seuls pour qui ce concept ne prendrait pas forcément sens seraient les jeunes ayant subi une migration qu'ils n'ont pas choisie et dont ils ne se sont pas approprié le projet ensuite. Ils sont peu nombreux. S'émanciper d'une situation non désirée en aspirant à un ailleurs serait donc le dénominateur commun pour la majorité de ces jeunes. S'émanciper et partir à la conquête d'une place. Une place tout court pour ceux qui n'en avaient pas, une place en accord avec les aspirations initiales pour les autres.

Afin d'étayer ces propos, arrêtons-nous quelques instants sur les projets de vie. Tous les jeunes rencontrés, sans exception, quel que soit le stade auquel ils se trouvent dans leurs parcours à l'Aide Sociale à l'Enfance, ont des projets. En accord ou non avec leurs possibilités et sur la base de niveaux d'information disparates, certes, mais des projets. Ces jeunes, donc, ont tous des envies et des aspirations. Même ceux qui ne souhaitaient pas partir et qui peuvent souffrir aujourd'hui du déplacement se mobilisent pour se former et acquérir un métier. Tous tentent de rendre constructif et bénéfique le projet migratoire. "Faire quelque chose, [...] s'autoriser soi-même à investir l'ici, en y construisant son devenir dans le maintenant ", migrer pour se sentir « complet, entier, abouti » (Fouquet, 2007). 
Francesco Vacchiano reprend De Boeck et Honwana en indiquant que les jeunes sont « les protagonistes d'un processus de signification du monde, de négociation de l'appartenance et de revendication de citoyenneté qui trouve dans l'accès à la mobilité l'une de ses dimensions les plus considérables " (2014). II stipule que derrière les conditions matérielles se dissimule un désir migratoire en lien avec les transformations sociales générées par le capitalisme. Le projet de départ prend forme sur la comparaison entre la situation vécue au pays et les représentations de ce que doit être le bien-être, entre conditions de vie réelles et projections imaginaires. Pour lui, la migration des jeunes est à mettre en lien avec la construction du sujet « moderne ». "Leurs histoires laissent voir une pluralité de situations et de parcours qui montrent que la migration autonome des mineurs a des raisons complexes et des dynamiques très différentes [...]. Cependant, un élément transversal renvoie à une série de modèles d'existence considérés comme souhaitables, en opposition à ceux expérimentés et expérimentables, dans son propre contexte de vie. » (Ibid.). Les jeunes sont attirés par l'espoir d'une vie "plus digne " et des modes de vie "plus modernes ".

"Moi, je ne voulais pas cela parce que ce que l'on cultive, c'est ce que l'on va manger. On mange quand même, mais cela ne bouge pas! C'est toujours comme ça! Tu cultives, tu manges, tu cultives, tu manges... Moi je voulais continuer mes études et après, travailler! Mais lui il ne voulait pas. "

Oussy, malien, 18 ans.

Selon Francesco Vacchiano (2014), ces modèles se fondent sur un « ailleurs imaginaire " lié à la possibilité d'accéder à un statut différent. Être moderne, c'est accéder à la société de consommation. "L'être au monde contemporain » repose sur des «modèles qui relient le statut de l'individu à sa capacité d'acquisition et de consommation, [...] à l'autonomie individuelle et à la subversion de l'ordre des générations " (Ibid.). La consommation confère un statut en ce qu'elle traduit des capacités. Elle offre la possibilité de se distinguer de ses aînés, de négocier son identité et de s'accomplir. Ne pas posséder, c'est se trouver « aux marges d'une citoyenneté matérielle ».

La mobilité ouvre ainsi sur des possibles. "Les jeunes aspirent au mouvement et au changement comme manière intrinsèque de participer au monde et comme possibilité d'être dans le temps. " (Ibid.). Si la société de consommation n'est pas accessible chez soi, on va la chercher ailleurs. Plutôt que de rester à l'écart, on fait le déplacement. L'univers des possibles dépasse les frontières. Les inégalités ne sont certes pas gommées, mais compensées. Partir, c'est lancer « un défi à un ordre social injuste. " "La migration vers l'Europe est perçue comme un acte compensatoire 
d'affirmation de soi ainsi qu'une façon de participer au monde en saisissant ses opportunités de réussite matérielle. " (Ibid.). La mobilité, associée à la réussite, s'impose face à l'immobilisme.

"Si on reste au pays, on aura des copains d'Europe qui donneront des choses mais ce sera la honte pour eux. C'est pour cela que les gens ne veulent pas rester là-bas sans travail. Ils veulent partir pour travailler. Il y a un vieux chez nous qui nous disait toujours : "Plutôt que de donner à la personne chaque jour du poisson, mieux vaut lui apprendre comment pêcher ». Il vaut mieux l'amener là où tu travailles et lui montrer. C'est pour cela que tout le monde quitte là-bas pour venir ici. "

Maamuni, sénégalais, 17 ans.

Dans son étude auprès des jeunes de Douala (Cameroun), Séraphin Gilles parle de la " conquête statutaire " qui s'opère à travers la migration. Parmi les statuts visés, être "sans souci " et " grand " renvoient respectivement au confort matériel et au prestige social. II explique que dans cette société hiérarchisée et statutaire, les jeunes oscillent entre soumission aux statuts générationnels et de sexe préexistant et stratégies pour se positionner et se faire une place. Avoir une activité, gagner ses propres ressources, acquérir un diplôme, développer un réseau social, ou encore adhérer à une religion, donnent accès à un statut. "Les migrations constituent l'une des principales stratégies historiques pour les habitants de Douala en quête statutaire [...]. Cette stratégie "des jambes" paraît une arme prometteuse à celui qui a le sentiment de « piétiner » (Séraphin, 2001).

Francisco Vacchiano met également en lien les possibilités " d'être ailleurs " avec le désir "d'être comme" (2014). Se rapprocher de l'Autre, physiquement par le déplacement, mais aussi dans ses modes de vie, par la promiscuité. Cette aspiration, Thomas Fouquet la place au rang de droit. "En niant les désirs de l'Ailleurs des candidats africains à la migration, pour ne s'attacher qu'à une interprétation molle de leurs besoins et nécessités matérielles et/ou économiques, c'est en fait une part de leur subjectivité que l'on dément. Enfermés dans des représentations où le thème de la misère (mais aussi du sous-développement, de la maladie et de la guerre ethnique) est omniprésent, ils n'ont pas droit de cité dans les sphères du désir d'émancipation et d'accès à l'Autre et l'Ailleurs. " (Fouquet, 2007).

"Ce sont les bonnes personnes que l'on vient chercher quand on se déplace. Pour avoir des idées, pour avoir des trucs. [...] A chaque fois que l'on s'approche de vous, on récupère des idées [...]. Cela nous aide toujours. "

Issan, guinéen, 18 ans. 
Nous avons vu que migrer visait l'accès au statut d'individu moderne. En considérant le phénomène sous cet angle, c'est la capacité du sujet à agir sur sa vie que l'on prend en compte. Nous allons voir cependant que selon les contextes, les marges de décision et de manœuvre sont plus ou moins étroites.

\section{TANTÔT ACTEUR, TANTÔT AGENT DU PROJET MIGRATOIRE}

\subsection{Projet migratoire et autonomie}

Emmanuel Ma Mung définit le projet migratoire comme « l'intention de quitter un lieu pour un autre et/ou l'accomplissement même de la migration avant que celle-ci ait atteint son terme " (2009). II reprend les propos de Florence Boyer (2005) : " Le projet migratoire doit s'envisager comme une « procédure stratégique, pragmatique et contextuelle " dont la fabrication est intentionnelle, une projection dans l'avenir ayant ainsi une dimension temporelle fondamentale dont le contenu, par là-même, est en redéfinition constante en fonction de l'histoire, des parcours et de l'expérience migratoires ". La temporalité est centrale dans la délimitation du concept de "projet migratoire ». Celui-ci est constitué de représentations et projections, se nourrissant des expériences et évoluant ainsi au fil du processus de migration. Une fois ce dernier achevé, le projet n'a plus lieu d'être et laisse place au vécu de la situation postmigratoire.

Pour Emmanuel Ma Mung (2009), en considérant la migration comme réalisation d'une intention, "ce ne sont plus des mécanismes qui agissent sur le migrant mais le migrant qui agit de lui-même ". II devient ainsi acteur de sa trajectoire en mobilisant ses propres ressources, qu'il met à l'épreuve du contexte l'entourant. "Le projet migratoire en tant que projection dans le futur est une façon d'organiser et surtout d'imaginer l'avenir. II apparaît dans la perspective autonome adoptée ici comme la formalisation des dispositions intérieures (initiative et savoir-faire) des sujets. Et se présente comme interface [...] entre dispositions internes et conditions externes, entre intériorité du sujet comme être-pour-soi et extériorité matérielle-sociale. »

La " perspective autonome " renvoie à " l'affirmation et l'initiative ". En effet, pour Emmanuel Ma Mung (2009), la migration traduit une forme d'autonomie dans la mesure où il y a " action délibérée, explicite, de sortir de son monde pour transformer sa situation et modifier le cours de son existence ". Face à un même contexte, certains choisissent de rester, alors que d'autres décident de prendre la route. " La migration consiste à quitter un univers social pour un autre, il y a donc comme une rupture du cercle social dans lequel on se tient. L'autonomie est ici accomplissement d'une action 
délibérée - délibérée étant à prendre dans ses différents sens d'action intentionnelle et d'action ayant fait l'objet d'un examen, d'une discussion. " Examen des gains par rapport aux coûts face à la différence de niveau de vie entre les pays d'origine et de destination ? C'est sûrement en partie le cas, mais pas uniquement. Cette vision économique de la migration est en effet réductrice et lorsqu'Emmanuel Ma Mung parle d'autonomie, c'est toute une posture qu'il met en avant. Quitter son pays et son " cercle social ", c'est une décision lourde de conséquences pour l'individu en tant que sujet. La prendre, c'est s'affirmer. En s'aventurant sur le chemin de la migration, les Mineurs Non Accompagnés agissent sur leurs vies et sur leurs trajectoires.

"Quand un jeune n'en peut plus, il part tout seul! Et il ne dit même pas qu'il va y aller. II prend sa décision et il part [...] Quand tu en as marre, tu es obligé de faire quelque chose!»

Oussy, malien, 18 ans.

Partir, donc, c'est selon Emmanuel Ma Mung faire preuve d'autonomie psychique, en assumant un choix que le sujet juge bon pour lui. Pour lui et/ou ses proches. Pourtant, le projet individuel parfois, résulte aussi souvent d'une décision familiale.

\subsection{Projet individuel versus projet familial}

Prendre un matin son sac à dos et partir sans prévenir personne, accepter un projet de départ proposé par la famille, suivre un oncle pour le Mali et comprendre en chemin que la route se poursuit vers l'Europe... Partir, fuir, subir, suivre... Partir... Les deux tiers des jeunes interrogés étaient volontaires à la migration (à l'initiative du projet ou en accord avec la décision), le souhait de suivre des études étant mis en avant comme principal motif de départ. Les autres ne voulaient pas quitter leurs pays et le départ a généralement été organisé par les personnes qui les recueillaient. II s'agit en grande majorité de " mineurs-éloignés ».

Nous pouvons supposer que le positionnement du jeune dans le projet migratoire a un impact sur le vécu de sa situation postérieure. Nous faisons ici référence aux deux positions énoncées par Angélina Etiemble et Omar Zanna, à savoir celles de "l'acteur" et de "l'agent ». Michel Crozier et Erhard Friedberg (1977) définissent l'acteur comme un " individu autonome, capable de calcul [...] et qui non seulement s'adapte, mais invente en fonction des circonstances et des mouvements de ses partenaires " (dans Dubar, 2017). L'acteur est à l'initiative du départ. Il mobilise les ressources dont il dispose pour atteindre ses objectifs. L'agent, entendu par Pierre Bourdieu (1994) comme " un individu pris dans la pratique et immergé dans l'action, 
agissant par nécessité » (Ibid.), subit la migration imposée par la famille ou la communauté. Décidé à partir, l'acteur serait prêt à affronter les obstacles. L'agent, quant à lui, se verrait d'autant plus fragilisé par les difficultés qu'il ne souhaitait pas prendre la route. Les récits suivants illustrent le vécu douloureux de la traversée pour des jeunes qui n'avaient pas choisi la migration et qui parfois n'avaient pas même été informés.

"Mon cousin avait payé. On a poursuivi la route qui était vraiment difficile. Mon cousin, quand même, il s'occupait de moi parce que lui, il est grand. II appelait le correspondant dans chaque ville. On est allé à Bamako. J'étais complètement découragé mais il criait sur moi. Il m'a pris ma puce de téléphone pour ne pas que j'appelle et que j'essaie de repartir. On a suivi la route mais je voulais me retourner. On a ensuite trouvé des campements de clandestins. II y avait la guerre. J'avais peur. " Ouma, guinéen, 17 ans.

"Quand je suis parti de Côte d'Ivoire, je ne savais pas jusqu'où l'on irait, je pensais qu'on s'arrêterait au Mali. C'est au Maroc que j'ai su qu'on continuait. Le Malien m'a dit de ne pas m'inquiéter et de ne pas avoir peur. C'était dangereux et difficile dans la forêt. Avant, je n'avais pas de projets. Comme c'était lui qui décidait, c'était sauver ma vie, étudier... Mais j'ai vu qu'on voyageait sans avoir de coin fixe pour étudier. J'ai compris qu'on se cachait et que ce n'était pas juste un voyage. "

Abdoulaye, ivoirien, 16 ans.

Joël Fronteau souligne l'importance de chaque phase du processus migratoire. "Ainsi, le fait de ne pas vivre pleinement l'une d'entre elles (surtout les phases prémigratoires, comme dans le cas de réfugiés ayant fui précipitamment) peut avoir de sérieuses conséquences par la suite, notamment sur l'adaptation. » (Dans Legault, 2000, p.3). Si un lien peut être fait entre décision de départ et vécu du parcours migratoire, notre étude ne permet pas d'en dire autant de l'adaptation postérieure à la société d'accueil. Les deux tiers des jeunes interrogés se disent à l'aise dans leur situation actuelle, contre un tiers qui expriment un mal-être (souvent du fait de ne pas être encore intégrés). Parmi ceux qui ne vont pas bien, la moitié était d'accord pour partir, et inversement, des jeunes n'ayant pas choisi la migration se sentent à l'aise et intégrés.

Julien Bricaud apporte une nuance pertinente en insistant non pas sur le consentement initial mais plus sur l'adhésion, même progressive, au projet : " Les jeunes qui arrivent seuls en territoire inconnu évoluent dans un décor étranger, loin de toute familiarité. La manière dont ils affrontent cette expérience varie selon la manière dont ils épousent le projet migratoire. » (2012, p.23). Le consentement au départ ne suffirait donc pas à faciliter ou à inhiber le processus d'adaptation, ce serait plus l'appropriation du projet qui aurait un impact. Vérifier ce supposé lien impliquerait 
d'interroger les jeunes après la phase d'installation, ce qui n'est pas le cas de notre étude puisque nous avons choisi de les interroger aux différentes étapes du processus.

Notre étude ne permet pas de vérifier le lien entre les modes d'organisation du projet (avec l'adhésion comme paramètre à prendre en compte) et l'adaptation à la société d'accueil. Pour revenir à notre problématique de départ, l'angle que nous privilégierons pour aborder la question des relations familiales sera celui du rôle social.

\subsection{Rôles vis-à-vis de la famille}

Quelles sont les attentes des familles à l'égard des jeunes qui prennent le chemin de la migration et comment réagissent ces derniers ? Doudou Dièye Gueye (2007) s'intéresse aux différentes formes de mobilisation dans les projets migratoires, indiquant que lorsqu'il y a soutien familial, les comportements ultérieurs du migrant s'en voient impactés. "Aux différents types de stratégies de départ répondent des formes distinctes d'exigences, mais également, sous certaines conditions, des formes d'implication diverses du bénéficiaire - émigré - envers la parenté, le groupe ou le réseau qui l'a soutenu ". Recevoir une aide à la réalisation de son projet implique donc de rendre la pareille une fois installé. Selon l'auteur, c'est en partie dans cette perspective que le réseau familial concourt à l'organisation des départs. Mais pour que ce "retour sur investissement " soit possible, le migrant doit, à son arrivée dans le pays de destination, être étayé dans ses démarches d'insertion. "Ce poids avéré de la famille dans le processus migratoire s'explique pour une large part par le fait que tout départ en émigration soutenu par cette institution contribue, à moyen ou à long terme, à alléger considérablement les charges liées au fonctionnement de l'unité de production domestique familiale en question, à la condition toutefois que l'immigré se trouve inséré dans un dispositif économique favorable dans le pays d'accueil. Par " dispositif favorable " nous entendons la possibilité de trouver dès son arrivée un réseau susceptible de l'encadrer et de l'aider et surtout de lui trouver un travail. " (Ibid.).

Dans le cas des Mineurs Non Accompagnés, la migration relève souvent de " mobilisations individuelles », sans aide financière, que Doudou Dièye Gueye juge des plus "intenses", en ce qu'elles mettent à contribution l'individu tout au long du parcours et une fois arrivé à destination si aucun réseau ne vient en soutien. Ce public ne disposant pas de réseaux familiaux sur place, ce sont les services de l'Aide Sociale à l'Enfance qui jouent le rôle de "dispositif favorable ", même si nous verrons que d'autres acteurs peuvent être impliqués. 
Que la participation à la mise en cuvre du projet migratoire induise une implication postérieure du migrant envers sa famille est cohérent. Ce lien ne ressort pourtant pas de façon aussi évidente dans notre étude. Si la question du financement du voyage n'a pas été évoquée, nous nous sommes intéressés à la façon dont le projet avait été pensé, à savoir avec ou sans l'adhésion de la famille. Sur les dix-sept jeunes interrogés partis avec l'accord des parents, seule la moitié déclare avoir l'intention de leur apporter plus tard une aide financière. A l'inverse, parmi les seize qui énoncent une responsabilité à l'égard de leurs proches, certains sont partis sans l'accord des parents. Un projet migratoire soutenu par la famille n'entraîne donc pas forcément de dette et inversement, que les parents y soient opposés n'induit pas nécessairement une absence de soutien de la part du migrant par la suite.

Dans tous les cas, toujours selon Doudou Dièye Gueye (2007), " un projet migratoire, même organisé par le réseau, peut servir les intérêts individuels. II s'agit alors d'«exploiter au mieux les potentialités et les occasions de concrétiser les réalisations individuelles qu'ils se sont fixées, tout en restant fidèles à l'éthique du partage solidaire ". Malgré le devoir de ne pas oublier les siens, l'opportunité de se réaliser individuellement est au premier plan.

Nous sommes donc loin du mandat familial que l'on suppose imposé tel un poids, lourd à porter. Comme nous le présentions précédemment, ce mandat n'est pas forcément explicite et verbalisé. La plupart des jeunes jugent naturel de soutenir leurs proches. Comme s'il s'agissait d'une norme culturelle et intégrée. Phénomène qui explique en partie les inquiétudes et les crispations autour des questions financières dans les services de l'Aide Sociale à l'Enfance. Si l'on demande à ces jeunes comment ils vivent cette responsabilité, ils la trouvent normale, dans le cours des choses.

"Maintenant, c'est moi qui suis responsable. Responsable de tout quoi! Peut-être que je vais leur envoyer de l'argent. Tous les étrangers qui viennent pensent comme cela. Maintenant, c'est moi qui vais aider mes parents. Pour eux maintenant, c'est bon. Ils ont fait leur travail, en s'occupant de nous, en nous donnant la nourriture... C'est bon, maintenant c'est moi! Ce n'est pas beaucoup pour mes parents! Imaginez, pendant neuf mois j'étais dans son ventre! Après, je suis né et jusqu'à mes 15 ans, ils se sont occupés de moi ! C'est beaucoup! C'est trop!" Walid, afghan, 18 ans.

Pour Abdelmalek Sayad, les transactions financières font figure de maintien du lien avec la famille, dans un contexte où "l'absence" est vécue comme une " trahison ". " D'où l'importance de l'argent envoyé à la famille, qui ne représente pas seulement le soutien matériel que l'on peut attendre de celui qui gagne sa vie (à l'étranger ou ailleurs), mais qui est d'abord cette justification immédiate de l'absence, 
cette "obligation " absolue que l'on a envers ses parents, mais aussi envers soimême. » (Dans Témime, 1999).

Enfin, un autre versant de la responsabilité vis-à-vis de la famille est l'injonction à la réussite. Nous l'avons évoqué au sujet de la "culture migratoire ", lorsque l'on repart au pays, c'est le succès qui doit transparaître, aux yeux des proches, mais plus largement, de la communauté. Le retour n'est donc envisageable que si l'on a réussi. «Bien qu'une fois parti, le jeune migrant puisse vivre des drames ou bien se trouver dans l'impasse, tout retour en arrière semble impossible, synonyme de dangers ou d'une honte qui le tuerait. L'échec du voyage n'est pas envisageable. " (Bricaud, 2012, p.23). Qu'en est-il des relations avec la famille durant le séjour dans le pays de destination ? Plus que d'illusionner les siens, ou même de ne pas les désillusionner, il s'agit de ne pas les inquiéter. Ne contacter les parents qu'une fois arrivé à destination, taire les difficultés, ne repartir que si les objectifs sont atteints. II est question ici de statut, certes, mais aussi de liens affectifs et du rôle protecteur d'un parent à l'égard d'un enfant. Cette protection ne peut plus être assurée à distance, alors pour préserver et éviter d'inquiéter, on préfère se faire silencieux.

"Je suis sorti pour un but, donc comme je n'avais pas eu ce but, je ne pouvais pas me retourner. Je préférais affronter la mort que de me retourner. "

Semei, ivoirien, 18 ans.

" J'attendais. Parce que je ne pouvais pas blesser. Vous, vous savez à quel point l'enfant a de l'importance pour sa mère. C'est sa mère quoi! Dès que tu lui dis un truc, elle ne dort pas. Moi, ma famille, elle n'était pas au courant de mon départ. Je n'avais pas envie de dire un truc et qu'ils tombent encore malades ou qu'ils s'inquiètent trop. Tu vois ? J'ai pris le temps... Plus d'un an avant d'appeler ma mère pour lui dire. Au foyer, on me donnait de l'argent pour l'appeler mais je ne le faisais jamais. Aujourd'hui, je n'ai même pas envie de parler avec la famille au bled. Je n'ai pas envie de les blesser en leur disant que ça ne va pas. Ils vont s'inquiéter pour moi. Elle est malade déjà, je le sais. Elle va s'inquiéter. Donc ça ne va pas. ॥

Issan, guinéen, 18 ans.

Réussir à travers la migration rappelle les déplacements du milieu rural à I'urbain, en France, à quelques nuances près. Dans son étude menée au milieu du vingtième siècle auprès d'une communauté rurale du Vaucluse, Laurence Wylie décrit la recherche d'opportunités des adolescents à l'extérieur de leur village, se rendant en ville pour suivre leurs études. "Si tout se passait bien à l'école technique des Sorgues, ils ne reviendraient jamais à Peyrane, sauf en visite. [...] Si la chance leur souriait, ils feraient la fierté de leurs familles et ils seraient en mesure de leur apporter une aide financière qui compenserait amplement les sacrifices qu'on avait consentis pour eux. [...] Les seuls qui y reviennent sont ceux qui ont échoué dans leurs études, c'est-à- 
dire qui reviennent déshonorés ". (Wylie, 1968, pp.125-126). II s'agissait pour ces jeunes de partir en quête d'opportunités que ne leur offrait pas leur village. Réussir et ainsi soutenir en retour la famille qui avait jusque-là aidé à la réalisation des projets. Contribuer par la même occasion à son prestige social. Tout échec était, comme pour les migrants d'aujourd'hui, source de déshonneur. Mais l'impossibilité décrite par notre public à repartir, ne l'était pas pour ces adolescents de Peyrane. Ils se sentaient certes déshonorés en cas d'échec, mais ils rentraient auprès des leurs. Ils avaient au moins cette garantie de pouvoir se replier et d'être accueillis.

Partir, c'est donc s'offrir la possibilité de réussir, pour soi, pour sa famille aussi, mais pour soi d'abord, avec des effets collatéraux sur son entourage. Cette dialectique de l'individuel et du collectif, nous allons le voir, est présente tout au long du processus migratoire. Durant la traversée, l'individu n'est qu'un parmi d'autres, agent d'une organisation sur laquelle il a peu de prise. Mais même au sein de ce " monde migratoire ", il peut retrouver sa capacité à agir en prenant les informations pour la suite.

\section{PRENDRE LA ROUTE OU LA SUIVRE}

\subsection{Immersion dans un " monde migratoire »}

Le phénomène de la migration dans sa double dimension spatio-temporelle produit des espaces intermédiaires entre les pays d'origine et de destination, avec une réalité qui leur est propre et une organisation interne spécifique. C'est tout un parcours qui se dessine entre les points de départ et d'arrivée et il se franchit rarement seul. D'étape en étape, au fil des rencontres se développe un réseau facilitateur du voyage. On est informé des routes possibles, des tarifs des passages, on est mis en relation. On intègre une organisation prédéfinie dont on est "l'agent ». Position qui vient contrebalancer celle $d^{\prime}$ " acteur » endossée par celui qui a pris la décision de migrer. Les Mineurs Non Accompagnés oscillent entre ces deux positions au cours de leurs trajectoires.

Le parcours migratoire, donc, fait des jeunes migrants des " agents " soumis à une organisation et à des règles sur lesquelles ils n'ont pas de prise et auxquelles ils doivent se soumettre. La fréquence de l'usage de verbes ou expressions à connotation passive est frappante dans les récits des trajets : " on nous a emmenés ", " on nous a dénoncés ", " on devait mendier ", "c'est eux qui décident ", " on peut se faire tuer ", " on m'a fait descendre du train ", "ils ne m’ont même pas arrêté », " c'est lui qui décidait ", "si tu pars, on t'attrape », " on était poursuivis », " on nous a mis dans le 
zodiac ", "il m'a envoyé à Nador", " on est venu nous chercher ", " on nous a entassés dans le pick-up ", "ils nous ont fouillés", "ils te tuent et ils te jettent à la mer ", " on a été évacués ", "ils ont trié les gens ", " on vous disperse ", " on vient vous chercher », " il criait sur moi », "la Croix-Rouge m'a pris », etc.

"Ils ne te disent pas que ce soir tu vas partir. Tu es là et tu attends. Dans la nuit, il vient et il te réveille. Il parlait et moi je ne comprenais rien. Le jour où il décide, tu pars. "

Oussy, malien, 18 ans.

"Quand tu as l'argent, cela dépend d'où tu veux traverser. Ils te le disent: la barrière d'un côté, l'eau de l'autre côté. Ça dépend! Si tu veux passer par l'eau, tu vas voir les passeurs! La barrière, il n'y a pas de passeurs là-bas ! La barrière, tu te démerdes tout seul!"

Redei, camerounais, 21 ans.

François raconte son parcours. "Nous les aventuriers " renvoie aux migrants, dont il fait partie. Il décrit la traversée du Niger, de l'Algérie, du désert... Les trajets en moto, en bus... Les dénonciations à la police. II explique aussi comment s'effectuent les transactions financières, le passeur finançant les voyages que chacun rembourse une fois arrivé à l'étape suivante, où attend un autre intermédiaire. Les menaces et la violence sont de mise. François évoque son « contrat » qui s'est terminé en Algérie. II a alors intégré un ghetto. II devait pratiquer la mendicité pour financer ses nuits et au bout d'un mois, il a été emmené au Maroc. Le train, les trajets à pieds, la poursuite par la police... Au Maroc, il n'a pas accepté l'aide des habitants par crainte d'être dénoncé. II a rejoint un autre ghetto, avec une nouvelle organisation... Les plus anciens étaient désignés comme chefs et il fallait les respecter, sous peine de recevoir des coups. Le récit se poursuit, du Maroc à l'Espagne, avec en toile de fond la confrontation à des systèmes organisés dans lesquels il s'agissait à la fois de se fondre et d'en respecter les codes pour espérer pouvoir franchir l'étape suivante. Maamuni décrit une organisation similaire.

"Au Maroc, c'était difficile. Je vivais dans la forêt. J'ai passé plus d'un an là-bas, sans sortir. C'est là-bas que j'ai eu les rastas parce que je ne pouvais pas me laver. C'était difficile aussi pour manger. Quand on te prenait comme capitaine, tu devais conduire les autres. Quand on se faisait attraper, on devait retourner dans la forêt et attendre que ceux qui étaient devant passent. Là-bas, tu n'avais pas l'esprit tranquille. Les grands nous disaient d'avoir du courage, que tout le monde était passé par là, qu'un jour viendrait. C'est eux qui décidaient quand tu partais. Les Arabes nous amenaient car ils connaissaient la direction. Ils trouvaient le matériel pour le zodiac et devaient tout monter. Le capitaine montait le dernier. S'ils ne voyaient pas les Marocains, ils attendaient de tomber sur les Espagnols et criaient pour être récupérés. Il fallait entrer dans l'eau internationale. Sinon, tu devais repartir au Maroc. " Maamuni, sénégalais, 17 ans. 
Emmanuel Ma Mung reprend les concepts d'Alain Tarrius (1989) de "territoires circulatoires " et de " mondes migratoires " pour désigner ces espaces intermédiaires construits à travers la migration. " Ces univers sociaux et territoriaux - ces « territoires circulatoires " comme il les désigne - correspondent à ce que nous pourrions appeler des " mondes migratoires " produits par les migrants, et à proprement parler, créés dans la migration. On pourrait alors parler de la création de mondes migratoires et porter notre attention sur cette " création migratoire » qui apparaît ainsi comme une autre dimension fondamentale du processus migratoire. Cette création migratoire est création d'univers de représentations, de normes, de codes, propres aux migrants, qui diffèrent, du fait de leur engagement dans la migration, de ceux des sociétés d'origine et de départ. " (Dans Ma Mung, 2009).

Soumis à ces contraintes extérieures, les migrants interagissent et participent aussi à ce système. Les normes ne sont pas uniquement celles qu'imposent les passeurs, d'autres émergent entre les migrants eux-mêmes, comme dans une microsociété. "Chacun pour soi là-bas ! » lance Ouma, tout en précisant que ce sont les gens qui l'ont convaincu de rester alors qu'il envisageait de rebrousser chemin. Ils réagissent par solidarité ou probablement par instinct collectif de survie, dans une organisation ou un faux-pas individuel peut mettre en péril la suite du parcours des autres. Il s'agit en quelque sorte de codes de conduite à respecter. Alain Tarrius parle de « co-construction - construction réciproque - du sujet comme migrant et du monde comme migratoire ", l'un façonnant l'autre et inversement (Ibid.).

Le parcours migratoire plonge, donc, dans un univers particulier dont il faut respecter les codes pour avancer. Les conditions de traversée sont rudes et impactent les migrants. Les vécus sont cependant subjectifs et si la plupart en ressortent fragilisés, certains mettent en avant l'expérience qu'ils en tirent.

\subsection{Une traversée facteur de fragilisation bien que formatrice}

Aucune question dans le guide d'entretien ne faisait référence au parcours migratoire qui n'était pas l'objet de notre étude. Il était essentiel qu'il n'y ait pas d'ambiguïté avec le protocole d'évaluation de la minorité qui passe par un récit détaillé de la traversée. Pourtant, certains jeunes en parlent pour évoquer l'impact sur leur vécu actuel. Ils se voient confrontés à des souvenirs traumatiques dont ils ne parviennent pas à se défaire et qui hantent le présent. 
"Tout ce que j'ai passé, tout ce que j'ai vécu, c'est toujours là. Tu sais, il y a des trucs qui ne passent jamais, que tu n'oublies jamais. Moi-même, j'ai envie de me débrancher de ça mais je n'y arrive jamais [...]. Je t'ai dit que j'avais peur de tout quoi ! J'ai peur, de tout, tout, tout... J'ai peur de rencontrer des personnes et d'avoir des problèmes, j'ai peur d'aller chez des potes, j'ai peur d'aller marcher et de voir quelqu'un... Bien que je sois bien logé, nourri, que je travaille... Ça ne va pas! Moi je suis très, très faible. Faible... J'ai peur de tout... Avant, j'avais peur, mais depuis que j'ai traversé l'océan pour venir, j'ai peur de tout! J'ai vu des personnes que l'on jetait sous l'eau... J'ai peur de tout, tout, tout... "

Issan, guinéen, 18 ans.

Agathe Benoît de Coignac et Thierry Baubet, dans une étude sur la santé mentale des Mineurs Non Accompagnés, indiquent une prévalence élevée de troubles psychopathologiques chez ce public. Outre les remaniements identitaires liés à la période de l'adolescence qui s'effectuent dans un contexte particulier pour ces jeunes isolés (aspect que nous développerons plus loin), les auteurs rappellent les événements traumatiques vécus durant le voyage (2013). Les hospitalisations en psychiatrie de certains de ces jeunes ou encore les suicides relayés par la presse confirment leurs fragilités. A la consultation transculturelle de l'association Mana, on explique que beaucoup de jeunes parviennent à laisser de côté les traumatismes, qui risquent cependant de resurgir à l'occasion d'événements de la vie. Une sortie en bateau par exemple, qui peut raviver brutalement les souvenirs de la traversée. Les psychologues invitent les professionnels à mettre des mots sur le vécu, à redonner du sens et de la cohérence, de la chronologie à l'histoire de vie. Le concept $d$ ' " endurance psychique " décrit cette capacité à écarter les affects pour utiliser sa pensée et ainsi s'ouvrir aux apprentissages, mais aussi à la relation. Ces jeunes qui " tiennent », mais jusqu'à quand?

La dureté du parcours amène à revoir les projections. Faire venir un proche, pourquoi pas, mais pas dans les mêmes conditions!

"Mes amis étaient passés alors que les frontières étaient encore ouvertes, ce qui leur avait pris un mois. Moi j'en ai mis huit. Je pensais que je pourrais porter le problème. Mais quand j'ai vu la misère pendant le trajet, d'avoir faim, soif, trop chaud ou trop froid, je me suis dit que c'était vraiment trop difficile [...]. C'est trop compliqué. On peut se faire tuer par les balles, les chiens ou les animaux. On arrive à des endroits où on n'a rien à manger ni à boire. Je ne ferais pas venir mon frère. Je ne veux pas qu'il tombe dans la misère. "

Farhad, afghan, 17 ans. 
"Plus tard, si je suis posé, pourquoi pas monter une entreprise. Pour les jeunes de là-bas, pour ne pas qu'ils se disent que comme Semei a pris l'eau, ils vont prendre l'eau aussi. Semei a eu de la chance de passer... D'autres n'ont pas eu cette chance et sont restés dans l'eau. Tout ça pour les encourager à travailler là-bas. Si vous voulez venir, ne me demandez pas parce que c'est très difficile. Si vous venez, c'est votre problème, mais moi je vous dis de ne pas venir. "

Semei, ivoirien, 18 ans.

Pour certains, faire la traversée, "comme les autres ", c'est aussi grandir. Apprendre, gagner en expérience. Traverser « en homme » nous dit Redei.

"Des fois c'était bien, des fois ça tournait mal. Mais Dieu nous a sauvés. Si tu sors, tu vas voir beaucoup de choses, du banditisme, de la criminalité. Cela développe l'expérience !» Ange, guinéen, 17 ans.

"La traversée, elle m'a beaucoup appris. Observer, calculer... Ça me sert aujourd'hui ! [...] Elle m'a fait grandir oui, mentalement, techniquement... »

Redei, camerounais, 21 ans.

La traversée est compliquée et laisse des traces chez les individus. Pour certains, elle fait grandir. Mais le "monde migratoire " est aussi le lieu où s'échangent les informations, permettant de préciser les stratégies pour la suite.

\subsection{Des niveaux d'information disparates}

Nous l'avons vu précédemment, la "culture de la migration" contribue à façonner les représentations sur l'Europe et nourrit le désir d'exil. Certaines formes de migration reposent en amont sur une connaissance accrue des contextes d'arrivée. C'est le phénomène qu'a pu observer Thomas Fouquet dans son étude sur les jeunes dakarois candidats à l'exil. " Le Nord est en effet connu de manière précise, tant par l'accès - souvent boulimique - aux nouveaux médias que par les discours sur l'Ailleurs proposés par ceux qui ont voyagé. Le projet de départ s'inscrit dans des stratégies très denses, agrégeant souvent une masse impressionnante d'informations qui tendent à le rationaliser autant que possible : potentialités d'être régularisé dans tel pays plutôt que tel autre ; de s'insérer dans un réseau de migrants déjà existant, plus ou moins enclin et apte à accueillir le nouvel arrivant ; bons plans et bonnes connexions sur place pour accéder rapidement à une activité rémunératrice, etc. Stratégies qui impliquent, en tout état de cause, une scrutation attentive des contextes sociaux, politiques, économiques, administratifs, etc. des sociétés du Nord, dans leurs diversités. » (Fouquet, 2007).

Pour les Mineurs Non Accompagnés que nous avons rencontrés, la réalité est tout autre. II s'avère que c'est principalement durant la traversée qu'ils reçoivent les renseignements concernant les pays de destination. Nombre de jeunes partent en 
effet sans idée précise de ce qu'ils vont trouver, ni même de ce qu'ils cherchent. Si une partie se projette spécifiquement en France, d'autres ne visent pas de pays en particulier. Le parcours migratoire vient agrémenter le projet en donnant accès à de nouvelles informations sur les différents pays, permettant de faire des choix stratégiques pour la suite.

"Je ne savais pas que je partais hein! Je partais juste pour partir! Dans ma tête, c'était I'Espagne. Après l'Espagne, au cours de la route, tu apprends de nouveaux trucs. Et tu allonges ton itinéraire, en France... D'autres partent en Belgique... Sur la route, tu rencontres des gens qui partent en Australie, en Suisse, en Belgique, au Danemark... Mon plan, c'était l'Espagne. Chez nous, les gens s'arrêtent en Espagne. Mais quand tu arrives là-bas, tu te rends compte que tu ne peux pas parler espagnol, que c'est dur, qu'il faut apprendre et tout, alors que le français, il est là ! Tu vois ? Il faut juste cadrer et tout mais... Aller où c'est facile plutôt que là où c'est difficile! J'ai des amis qui sont allés en Allemagne car là-bas, on te donne l'asile et trois-cents euros... Mais c'était dur la langue. Donc j'ai choisi le plus facile, qui va vite ! Tu vois ? Le français. Voilà !»

Redei, camerounais, 21 ans.

Redei explique aussi que c'est durant le trajet qu'il a entendu parler de l'Aide Sociale à l'Enfance qui facilite "l'intégration » et " l'obtention des papiers » en France. Mais hormis un seul jeune qui avait eu ces mêmes échos, les autres énoncent avoir découvert l'existence des foyers lors de leurs premiers accueils en Europe, pour la plupart en Espagne et en Italie. Les niveaux d'information s'avèrent très variables, certains comme Redei étant au fait des dispositifs avant leur arrivée, grâce à leurs réseaux, alors que d'autres, plus isolés, sont moins renseignés et donc peu à même de faire valoir leurs droits. C'est l'exemple du jeune gambien, Issam, rencontré au Squid, installé à l'écart du groupe dans la pièce principale. Sa nationalité étant peu représentée, il n'a pas de compatriotes et personne ne partage sa langue. Sa maîtrise du français est selon lui trop limitée pour qu'il s'autorise à aller vers les autres. Il est arrivé à Bordeaux il y a un mois et au squat quelques jours plus tard. II n'est pas informé des associations servant les repas, où se rendent pourtant tous les autres jeunes, selon une organisation hebdomadaire précise. Au SAEMNA ${ }^{34}$, il y est "déjà passé une fois " mais " c'est fini ». II n'a pas été évalué, a seulement été inscrit (dans l'attente d'une place) mais il a compris que la prise en charge lui était refusée. II s'informe au fur et à mesure mais n'a pas encore une connaissance globale de son environnement et des dispositifs, si bien qu'il n'y a pas accès, au risque de passer à côté de ses droits.

\footnotetext{
${ }^{34}$ Service d'Accueil et d'Évaluation des Mineurs Non Accompagnés.
} 
A travers la constitution des réseaux migratoires, les informations s'échangent, mais tout le monde n'y a pas accès de la même manière, ce qui impacte les conditions futures d'installation.

\section{CONCLUSION DE LA PREMIÈRE PARTIE}

Pour appréhender l'expérience que font les Mineurs Non Accompagnés de la migration, nous avons exploré en premier lieu les contextes de départ. Nous avons été interpellée par la proportion de "mineurs-aspirants" et par le repérage d'une nouvelle catégorie: les "mineurs-éloignés". La notion de mandat implicite a également été une découverte. Nous avons choisi toutefois de nous décaler de ces typologies pour nous recentrer sur le sens donné à ces mobilités. La migration comme moyen d'accéder au statut d'individu moderne, telle est la dimension commune qui ressort des différents témoignages. Même lorsque le projet est familial, il vise l'émancipation d'un contexte de vie indésirable. Soutenir en retour la communauté ayant facilité le départ n'est pas incompatible avec la réalisation de ses intérêts personnels. Entre individu et collectif, liberté de décision et soumission à des contraintes extérieures, tels sont les va-et-vient opérés par ces acteurs capables de mobiliser les ressources à leur disposition pour aller de l'avant. La traversée en est une illustration. Nous allons voir en suivant comment sont vécues la rencontre avec les institutions et l'installation dans le pays d'accueil. 


\section{PARTIE 2. RENCONTRE AVEC UNE SOCIÉTÉ D'ACCUEIL EXIGEANTE}

\section{DES DROITS ET DES DÉMARCHES}

\section{1. "Les papiers, il n'y a pas ça chez nous ! »}

Si elle n'est pas nommée de cette manière, la " paperasse administrative " surprend et revient régulièrement dans les discours. Vingt-et-un jeunes insistent sur ce décalage avec les pays d'origine. "Pour aller chez le médecin, il faut la carte vitale ! " «Pour prendre le bus, il faut l'abonnement ! " "Dans la rue, il y a tout le temps des contrôles!» "Pour s'inscrire à une formation, il faut présenter sa pièce d'identité ! » « Là-bas, on va directement à l'école, comme ça ! »

"Les papiers, c'est chiant à faire. Les factures, c'est difficile à gérer. C'est dur les papiers, ça change tout le temps, il y a trop de choses à remplir ! Tout le temps, il faut remplir ça, il faut remplir ça... J'en ai marre! J'aimerais juste avoir un endroit où tu pars et tu coches. Pas là-bas, là-bas, là-bas... Trop de papiers, trop de factures à gérer... Nous on n'est pas nés dans ça, chez nous, au bled! Tous ces papiers-là, tous les jours des papiers... Chez nous, il n'y a pas ça!"

Redei, camerounais, 21 ans.

"Je ne savais pas que l'on ne pouvait pas travailler sans les papiers. Travailler, cela n'a rien à voir avec ça!»

Oussy, malien, 18 ans.

"J'ai fait un apprentissage mais pas comme en France, hein, en Algérie, au noir, pas avec les papiers et tout ! Direct l'apprentissage!"

Hamidi, algérien, 16 ans

Concernant les démarches auprès de la préfecture, elles requièrent la présentation d'un acte de naissance que la plupart n'ont pas en leur possession, voire qui n'a jamais été établi. En tentant de répondre aux exigences de la société d'accueil, les Mineurs Non Accompagnés se confrontent aux administrations de leurs pays et expérimentent les écarts, qui viennent souvent faire obstacle à leurs démarches. L'intermédiaire entre les deux États, c'est le consulat. Les formalités administratives y sont fastidieuses et incertaines. L'accueil n'y est pas toujours des plus agréables. Vécu douloureux pour les jeunes qui ont le sentiment de ne pas pouvoir s'appuyer sur leurs autorités. Alors que l'enjeu est de taille !

Prendre aux aurores le premier train en direction de Paris, se retrouver nez à nez avec une affichette indiquant une fermeture exceptionnelle, faire la queue durant deux heures sur le trottoir sans garantie de pouvoir entrer, passer de guichet en guichet en quête d'informations souvent contradictoires, attendre, avoir rendez-vous à 
9h00 auprès d'un service qui n'ouvre qu'à 9h30, s'entendre dire que le dossier a été perdu, voir circuler des billets de main en main, apprendre un mercredi que les légalisations ne se font que le mardi, attendre mais dans la cour par temps de canicule, se faire récriminer car ce n'est plus l'heure des questions, repayer la formalité car le dossier est bloqué mais " ne vous inquiétez pas, vous serez remboursé ", s'aventurer dans les toilettes sans lumière, attendre debout car il y a en tout et pour tout six chaises pour quatre-vingt-dix-sept personnes, constater une erreur dans l'orthographe de son nom et attendre de nouveau, manquer de rater son train retour après six heures passées sur place... Le rater. Attendre qu'on nous rappelle pour nous informer de l'arrivée du passeport, quand le numéro a été copié sur un mini post-it intercalé entre les quatre-vingt-dix-sept dossiers... "Mais repassez au cas-où, cela vaut mieux!»

A 9h00, dossiers à la main, les gens affichent un sourire, se rencontrent, discutent, dans leur langue ou en français, paisiblement. On croise çà et là des Mineurs Non Accompagnés avec leur éducatrice, la seule Blanche, avec son sac à dos, discutant du pays d'origine et des différences culturelles. A $11 \mathrm{~h} 00$, on s'impatiente. Puis, la phrase fatidique : «Ils sont mal organisés ! » Ou encore : " Avant, c'était la merde ici, mais maintenant, c'est la mort ! » Le ton monte, les visages se crispent, on se bouscule pour pouvoir entrer. Si l'on peut, on passe devant celui avec qui l'on ironisait sur son pays. On ressort soulagé ou au contraire remonté contre ses autorités, angoissé. Il est $15 \mathrm{~h} 00$, on n'a pas mangé. Détail important, au consulat, on ne mange pas! On attend d'être devant la tour Eiffel, après la séance photos.

Un espace-temps à la marge, un entre-deux: entre deux pays, entre deux cultures, entre deux systèmes administratifs. On est ambivalent. Attaché à son pays, en colère aussi. Contre qui ? Contre ses autorités qui tardent et peinent à établir les documents d'identité ? Contre celles de la société d'accueil qui les contestent ? «On va se naturaliser sénégalais " ironisent des jeunes guinéens las de patienter au consulat, alors qu'à quelques stations de métro de là, leur camarade sénégalais a récupéré son passeport deux heures seulement après avoir déposé la demande.

"Pour nous, les Guinéens, c'est très difficile parce qu'on ne fait pas les passeports. Je me dis que notre gouvernement est très faible. II ne met pas les moyens à disposition de la population. Une ambassade qui ne fait pas les passeports, c'est difficile ça! L'ancien président de la transition a dû prendre la nationalité sénégalaise pour avoir un passeport parce que le sien était expiré. Si à lui on lui a infligé ça! Nous, le bas peuple, comment on va faire? Quand tu appelles, l'ambassade ne te répond pas! C'est bizarre ça! La Guinée ne donne pas de passeports depuis 2017. Ce n'est pas grave ça?"

Ange, guinéen, 17 ans. 
Face à la complexité des dossiers administratifs, les jeunes interrogés expriment la nécessité d'être soutenus. Bien que ces démarches génèrent une forme de dépendance à l'égard des professionnels, ils savent qu'elles ouvrent accès à des droits. Scolarisation, soins gratuits et aides sociales en France sont des éléments qu'ils valorisent, en comparaison avec les systèmes existant dans les pays d'origine. C'est le gage d'une stabilité future. Il faut en passer par là !

"Si tu n'as pas d'éducateurs, tu ne t'en sors pas! Ou il faut vraiment avoir du monde que tu connais ici! Même si tu parles la langue, tu n'as pas le temps d'aller au lycée, de travailler et de faire les démarches!"

Anton, kosovar, 25 ans.

"Là-bas, quand tu es malade et qu'il faut aller à l'hôpital, tu crois que l'État prend soin de toi ? Non! Avoir des aides, jamais de la vie! L'argent que tu vas envoyer, c'est le traitement que l'on va te donner. Si tu n'as pas d'argent, c'est fini pour toi ! » Issan, guinéen, 18 ans.

"Chez nous, là-bas, on dit qu'un papier ne ment pas. Si c'est mentionné, c'est mentionné ! Si tu viens avec tes papiers, on dit que c'est toi. Cela aide, vraiment !" Maamuni, sénégalais, 17 ans.

Des papiers, donc, et encore des papiers! Un décalage avec le pays d'origine, certes. Des démarches complexes pour lesquelles un coup de pouce n'est pas de refus! En arrière-plan, la possibilité d'accéder à des droits. Mais des droits sous conditions.

\subsection{Un accès spécifique au droit commun}

L'accès aux droits en France constitue le début de l'intégration. Être comme les autres, mais aussi faire reconnaître son rôle en tant qu'acteur social. Cotiser, déclarer ses revenus, demander une aide au logement ou encore bénéficier d'une couverture maladie, c'est une façon d'exister. Abdelmalek Sayad, au sujet des immigrés algériens dans les années 70, évoque une " nouvelle identité sociale " qui s'impose au contact de l'organisation sociale du travail, " une autre manière de se définir, une autre représentation de soi ». II précise que ce sont les fiches de salaire qui symbolisent "leur nouvelle condition d'ouvriers ou plus exactement d'émigrés «installés» dans la condition de l'émigré " (Sayad, 1977). Ces bulletins de paie donc, sont plus que de simples papiers. Ils ont à voir avec l'identité. Être « comme eux ». 
Pourtant, pour accéder aux droits en France, toute personne étrangère (hors Union européenne) est soumise à une condition de séjour.

"En Europe, si tu n'as pas de papiers, tu es nul, ça ne sert à rien, tu ne vis même pas. Parce que si tu n'as pas de papiers, tu ne vas pas travailler, tu ne peux pas prendre de maison... En Afrique, tu t'en fiches des papiers, que tu travailles ou pas, tu n'as pas besoin de papiers! En Europe, il faut des papiers!»

Ouma, guinéen, 17 ans.

Même détenteur d'un récépissé, s'il s'agit d'une première demande de titre de séjour, on ne peut avoir droit aux prestations de la $\mathrm{CAF}^{35}$, ni accéder à un logement. On se trouve donc en situation régulière en France mais avec des droits limités. Sans titre de séjour, ni récépissé, on peut prétendre à une couverture maladie au titre de $\mathrm{AME}^{36}$. Un droit donc, mais spécifique. Les Mineurs Non Accompagnés ayant bénéficié de la $\mathrm{CMU}^{37}$ le temps de leur prise en charge dans les services de l'Aide Sociale à l'Enfance conservent quant à eux ce droit à la majorité. Signe qu'ils sont davantage inclus dans la société. Quid de la carte vitale ? Fréquemment, elle se perd dans les méandres des administrations et tarde à arriver jusqu'à eux. Bien que l'attestation suffise pour accéder aux soins, ils insistent et s'insurgent contre une défaillance des institutions. A travers cette bataille, c'est une place dans la société qu'ils semblent rechercher.

Concernant l'insertion professionnelle, le titre de séjour ouvre accès à l'emploi mais de façon restrictive puisqu'il est soumis à une autorisation de travail délivrée par la DIRECCTE ${ }^{38}$. De nouveau, il faut en passer par de nombreuses démarches administratives pour faire valoir ses droits et elles peuvent constituer un frein à l'embauche.

"Quand le patron veut signer le contrat, le moment fatidique arrive : le dossier! II faut aller à la chambre des métiers, rapporter tel papier, il faut que le patron présente telle facture qu'il avait gardée, qu'il déclare... Mais certains patrons ne veulent pas afficher tous ces trucs. Et ça bloque le contrat! T'es baisé ! En plus, il y a des taxes à payer. N'importe quoi ! On doit payer des taxes pour travailler?"

Redei, camerounais, 21 ans.

Au sujet des Mineurs Non Accompagnés, Marie-José Pagnon souligne l'impact de la loi du 26 novembre 2003 sur leurs modalités d'installation. Alors que tout mineur confié aux services de l'Aide Sociale à l'Enfance pouvait jusqu'alors acquérir la nationalité française à sa majorité, sur déclaration auprès du tribunal d'instance et

\footnotetext{
${ }^{35}$ Caisse d'Allocations Familiales.

${ }^{36}$ Aide Médicale d'État.

${ }^{37}$ Couverture Maladie Universelle.

${ }^{38}$ Direction Régionale des Entreprises, de la Concurrence, de la Consommation, du Travail et de l’Emploi.
} 
sans condition de durée de prise en charge, ce critère a été réintroduit et porté à trois ans. "Le rapport nationalité française / installation s'est inversé : il faut aujourd'hui qu'un jeune migrant prouve son installation sur le territoire pour obtenir la nationalité française ". Son statut est lié à son activité, qu'elle soit scolaire ou professionnelle, alors qu'avant la loi de 2003, l'attribution de la nationalité française était considérée comme « un moyen privilégié d'aide à l'installation et à trouver une place sur le territoire français » (Pagnon, 2011).

Les lois en matière d'immigration renvoient à la conception que l'on a de l'Étranger et à la place que la société souhaite lui donner. " Les regards et imaginaires occidentaux sur l'Afrique et ses ressortissants immigrés [...] déterminent partiellement les places octroyées aux migrants et/ou sollicitées par eux au sein des sociétés d'arrivée à travers le prisme de la construction de l'altérité. " (Fouquet, 2007). Lorsque l'on parle de " place octroyée aux migrants ", on ne s'intéresse plus aux rôles joués par ces acteurs mais à la volonté ou non du pays de destination de les accueillir.

Abdelmalek Sayad développe le concept de "pensée d'État », qui détermine la représentation du monde. "Le phénomène migratoire en sa totalité, émigration et immigration, ne peut être pensé, ne peut être décrit et interprété, autrement qu'à travers les catégories de la pensée d'État. Ce mode de pensée est tout entier inscrit dans la ligne de démarcation, invisible ou à peine perceptible, mais dont les effets sont considérables, qui sépare de façon radicale "nationaux » et " non-nationaux » [...]. » (Sayad, 1997). Discriminer est selon l'auteur dans la nature-même de l'État, ce qui se traduit par l'imposition de cette différence de statuts.

Faire valoir ses droits passe par des démarches administratives et pour les personnes étrangères, les circuits sont parfois spécifiques. Pour accéder aux services de l'Aide Sociale à l'Enfance et au statut de Mineur Non Accompagné, il faut justifier de son identité, mais aussi se soumettre au protocole d'évaluation. Pour certains, c'est le parcours du combattant.

\subsection{Une évaluation de la minorité légitimée mais contraignante}

En France, quel que soit le département, l'évaluation de la minorité est une étape incontournable pour toute personne étrangère souhaitant demander une prise en charge au titre de l'Aide Sociale à l'Enfance. Elle consiste généralement en différents entretiens visant à recueillir les récits de vie et à en mesurer la cohérence. D’après ceux qui ont transité par l'Espagne, le processus y serait plus souple, fondé 
essentiellement sur du déclaratif, même si les documents administratifs seraient de plus en plus souvent demandés.

Quinze jeunes s'expriment sur les exigences liées à la procédure, se trouvant pour la plupart dans cette phase d'évaluation ou en début de prise en charge. La moitié déclare trouver "normale " l'évaluation. Que l'on cherche à savoir qui ils sont avant de les accueillir leur semble logique et ils jugent pertinent de privilégier la prise en charge des mineurs à celle des majeurs, ces derniers ayant d'autres possibilités. Apporter la preuve de son âge leur apparaît donc nécessaire.

"A Poitiers, on ne te prend pas si tu n'as pas de preuve comme quoi tu as l'âge, comme quoi tu es mineur! Je n'avais aucun document! On aurait dit que j'étais tombé du ciel! C'est moi qui ai fait le con!"

Osei, guinéen, 16 ans.

En revanche, plusieurs jeunes disent ne pas comprendre la procédure ou la remettent en question. Pour l'un d'entre eux, qui se trouve en phase de recours, ne pas reconnaître sa minorité et donc lui refuser la prise en charge n'est pas légitime de la part de professionnels qui ne savent rien des réalités de parcours et de la mer. Les critères administratifs ne font nullement pour lui le poids face à la souffrance endurée. D'autres trouvent surprenante la nécessité de devoir apporter la preuve de leur âge. Pour eux, le déclaratif devrait suffire et même s'il arrive que l'on paraisse plus âgé, l'aspect physique peut être trompeur. Pour étayer ce propos, il est important de préciser que la notion d'âge administratif ne prend pas forcément sens dans toutes les sociétés, thématique sur laquelle nous reviendrons un peu plus loin. Enfin, certains jeunes trouvent paradoxal qu'une fois fournis les documents exigés, leur authenticité soit contestée.

"Ici, quand on est mineur, on te prend en charge ou je ne sais pas quoi. Mais il faut des preuves. C'est ça que j'ai compris. C'est bizarre ! Moi je trouve que ce n'est pas logique mais c'est leur boulot. On ne peut rien dire mais ce n'est pas logique. Tu ne peux pas venir ici et mentir, dire que tu as 15 ans, cela n'a rien à voir en fait. " Sekou, malien, 16 ans.

"Je me suis dit que c'était bizarre. Quand tu n'as pas de papiers, on te dit non, tu n'as pas de papiers. Quand tu as des papiers, on te dit non, tu es majeur.» Semei, ivoirien, 18 ans.

"On m'a dit que l'acte de naissance n'était pas bon. Je leur ai demandé de me prouver qu'il n'était pas bon! Je ne ramène pas un autre! On ne fait pas deux actes de naissance dans la vie! J'en ai un seul!"

Redei, camerounais, 21 ans. 
Ces témoignages pourraient laisser penser que deux postures s'opposent face à l'évaluation, certains la légitimant alors que d'autres la contesteraient. II semble pourtant que ce qui se dessine entre les lignes est une distinction entre une vision théorique des procédures et un vécu subjectif. Si les Mineurs Non Accompagnés sont en mesure de comprendre les exigences que pose la société d'accueil, se confronter à ces obstacles administratifs est douloureux et source d'angoisse. En toile de fond, ce sont les enjeux liés à l'évaluation qui transparaissent et l'appréhension face à l'absence de solution alternative en cas de refus de prise en charge. Nous allons voir justement ce qu'attendent les jeunes des services éducatifs.

\section{DES ATTENTES ÉVOLUTIVES ENVERS L'AIDE SOCIALE À L'ENFANCE}

\section{1. Être étayé dans un contexte inconnu}

Tous les jeunes interrogés sur la question affirment qu'ils n'avaient aucune idée, avant d'arriver en Europe, de ce qu'était un éducateur. Chez eux, " ce sont les parents qui éduquent ". Ils relatent que les foyers là-bas n'accueillent que les enfants handicapés, les orphelins, ou ceux qui ont des troubles du comportement. " $A$ leur arrivée, les notions de foyer ou d'éducateur ne signifient rien pour eux " confirme Julien Bricaud (2012, p.79).

"Je n'attendais rien! Je ne connaissais pas l'ASE ${ }^{39}$ hein! J'attendais juste qu'on m'oriente dans la vie, qu'on me prépare un peu. "

Redei, camerounais, 21 ans.

Ce constat vient infirmer en partie notre première hypothèse portant sur les exigences de la société d'accueil qui seraient en décalage avec les représentations et les attentes de Mineurs Non Accompagnés. Nous supposions que les jeunes avaient connaissance du dispositif de l'Aide Sociale à l'Enfance avant d'arriver. Nous pouvions d'ailleurs nous demander si les possibilités de prise en charge en France pouvaient conditionner leurs projets de départ. Or, il s'avère que ces informations ne sont pas connues dans les pays d'origine (ou si elles le sont, elles ne sont pas mises au premier plan). Certes, durant le trajet migratoire, certains entendent parler du dispositif, mais ils sont en minorité. Lorsqu'ils découvrent les foyers à leur arrivée en Europe, ils n'ont qu'une vague idée de ce qu'ils peuvent être dans les autres pays. II semble que ce soit surtout après, une fois dans les services, que s'échangent (et parfois se transforment) les informations. Peuvent alors émerger effectivement des décalages entre les récits faits par les pairs et les fonctionnements trouvés sur place. Comme c'est le cas pour les questions financières. Par contre, même s'il n'y a pas au départ d'attentes envers le

\footnotetext{
${ }^{39}$ Aide Sociale à l’Enfance.
} 
dispositif de l'Aide Sociale à l'Enfance méconnu, il y en a bel et bien à l'égard des sociétés européennes, nourries par l'imaginaire migratoire. "On pense qu'on ramasse l'argent par terre » nous dit François. Ce qui impacte forcément le rapport aux services d'accompagnement.

"Maintenant je comprends un peu mais pas tout. Je ne sais pas d'où sort l'argent, je ne sais pas d'où il sort. Je trouve cela bizarre. Mon éducatrice m'a dit que c'était les impôts. J'ai dit oui mais c'est bizarre. "

Oussy, malien, 18 ans.

"Tu es là, tu as trois repas par jour, tu manges trois repas par jour ici. Chez toi, tu ne manges pas trois repas. Ici, tu ne sais pas comment font les gens pour avoir l'alimentation, pour que tu aies à manger. Chez toi, tu sais comment ton père travaille pour avoir ton alimentation. [...] Tu as I'alimentation, tu as les trucs qu'il faut, tu as des habits, on t'achète des habits. [...] Si on te dit qu'on est là pour te donner tout ça, tu vas dire quoi à ton avis? Tu vas dire que tu vas quitter l'Afrique pour rentrer au paradis ou pas?"

Issan, guinéen, 18 ans.

C'est donc en Europe que les Mineurs Non Accompagnés découvrent l'existence des foyers. Si être suivi par des éducateurs peut leur sembler étrange au début, la plupart le vivent comme une chance.

"On m'a expliqué que les éducatrices étaient là pour nous aider, comme nos parents, et qu'il fallait les écouter. J'ai dit ok. Au début, c'était difficile, mais avec le temps ça passe. Ce que je voulais faire, elles ne voulaient pas. Mais je ne pouvais rien dire car je ne les connaissais pas trop. Elles ne me laissaient pas sortir le soir. Je leur ai dit qu'un jour, je partirais du foyer et qu'elles allaient voir. Mais je suis parti et ça va. Même maintenant, je vais au foyer et on parle ensemble."

Walid, afghan, 18 ans.

Dans son étude sur les trajectoires post-institutionnelles de Mineurs Non Accompagnés à Lille, Émilie Duvivier (2010) analyse l'impact de la prise en charge dans les parcours de ces jeunes. Elle met en évidence le lien entre la concrétisation des démarches d'insertion et la poursuite ou non de la mobilité. L'Aide Sociale à l'Enfance apparaît comme une étape dans le parcours. L'auteure relève des départs en cas d'obstacles, induits par une perte de confiance envers les institutions qui perdent leur caractère sécurisant. Elle s'interroge sur une possible instrumentalisation du dispositif en constatant que certains mettent un terme à leur prise en charge une fois les titres de séjour obtenus. 
L'institution facilitatrice des projets, tel est le rôle que lui confèrent les jeunes ayant fait une demande de prise en charge. Des attentes qui évoluent au fil de I'installation, nous le verrons. Avoir un toit et aller à l'école, puis signer un contrat et percevoir ses propres ressources, être régularisé à 18 ans, pour enfin accéder au logement autonome. Mais pas uniquement. On entend parler çà et là de logique utilitariste. Pourtant, le recours à un quelconque dispositif ne vise-t-il pas la mise en œuvre de ses projets, quels qu'ils soient ? Des jeunes qui demandent l'arrêt du suivi une fois leur situation administrative régularisée, il en existe probablement. Comme d'autres, pas forcément de nationalité étrangère, qui mettent un terme à la prise en charge lorsqu'ils accèdent à leur premier appartement.

Les quatre jeunes accueillis en squat le temps de la procédure de recours judiciaire se trouvent comme dans un entre-deux, dans un espace-temps en suspens. Ils expriment chacun à leur manière un certain mal-être. Alors que Bayou se décrit comme étant "loin de la société " et "pas encore intégré ", Vinza explique ne pas avoir encore "les conditions " et voudrait "vivre bien". Saïd est las de " ne rien faire " et que "rien n'avance ". Lumumba subit également cette monotonie et ne se sent "pas tranquille ». Le champ sémantique de l'abandon est très présent dans les discours : "ils m'ont laissé ", "ils m'ont lâché », " pas assez aidé "... Ces mots font écho aux expériences antérieures de séparation d'avec la famille, de deuil, d'abandon parfois, mais aussi de manque de soutien ou d'opportunités dans le pays d'origine. Derrière la demande de prise en charge, c'est toute la question de l'accueil qui émerge, avec la possibilité de se voir octroyer une place, d'être entouré et porté, pour enfin se poser.

Les professionnels s'interrogent souvent sur les besoins éducatifs des Mineurs Non Accompagnés. De par leurs parcours, ils ont pu acquérir une certaine maturité et semblent parfois "autonomes». Mais pas nécessairement dans tous les domaines. Qu'en est-il de l'appréhension de ce nouvel environnement et de ses codes ? Le rôle d'un éducateur n'est-il pas de faire tiers, de médiatiser, d'amener vers l'autre et les institutions ? Les jeunes rencontrés expliquent demander une prise en charge car ils se trouvent seuls et ne connaissent pas le pays d'accueil et ses fonctionnements. Ils souhaitent apprendre « comment se comporter».

"Quand je suis arrivé en France, si je n'avais pas eu les éducatrices, je me demande comment j'aurais pu m'en sortir! Tout ce qu'elles font avec moi... Elles m'aident, m'éduquent aussi. Elles me donnent des cours, me disent les réalités en France... Elles nous apprennent les lois, ce que les Français veulent et ne veulent pas. " Maamuni, sénégalais, 17 ans. 
"L'éducatrice, elle est là pour te remonter le moral, te parler, t'orienter, te montrer comment faire les dossiers, pour demain quand elle ne sera plus là. C'est comme une présortie. Comme si elle te préparait au bac quoi ! Savoir comment gérer le monde dehors, savoir comment est la vie dehors en France, savoir comment les gens se comportent, comment se comporter avec les gens, comment s'exprimer surtout, très important! Ce n'est pas le même langage que nous, d'où l'on vient ! "

Redei, camerounais, 21 ans.

Être «pris en charge » signifie avant tout être accueilli, mais aussi soutenu et orienté dans un environnement méconnu. Ce sont les premières attentes des jeunes à l'égard des services de l'Aide Sociale à l'Enfance. Nous allons voir que rapidement, enclencher les projets d'insertion devient une priorité.

\subsection{Enclencher les projets d'insertion et s'intégrer}

Les jeunes rencontrés durant la phase d'évaluation énoncent tous de bonnes relations avec les professionnels et voient leurs besoins primaires satisfaits. Malgré tout, seuls deux d'entre eux disent se sentir bien dans le service, "comme en famille " précise Asad. Les autres mettent en avant un vécu difficile, du fait d'être inactifs ou de ne pas comprendre ce que l'on attend d'eux. Ne pas être scolarisé est peu entendable. Ils souhaitent que s'enclenchent les projets. Certains manifestent aussi leur incompréhension face à des contraintes extérieures qui les dépassent.

"On dirait que je suis prisonnier! Je mange, je dors. Je ne fais rien! Je mange, je dors! Je ne fais rien!"

Osei, guinéen, 16 ans.

" J'ai envie d'aller à l'école! Rester à l'hôtel, sans rien faire... Tu manges, tu dors, tu vas aux cours trois fois par semaine, mais ça ce n'est pas l'école! Tous les jours, on me dit que j'attends une place dans un foyer mais je ne vois pas de place! Ils m'ont dit qu'il n'y avait pas de place libre. Je ne sais pas, je ne comprends rien! Je ne comprends pas $"$. Hamina, guinéenne, 17 ans.

La barrière de l'évaluation franchie et les besoins vitaux satisfaits, on pourrait s'attendre à un apaisement et à un discours plus positif. Pourtant, plus de la moitié des jeunes se trouvant dans la première année de prise en charge ne se sent pas bien. Vécu d'isolement, manque d'intégration et incompréhension de l'environnement, mais aussi des attentes à leur égard, semblent en être les principaux motifs. Un jeune (que nous n'avons pas mentionné dans l'échantillon) a dû renoncer à l'entretien qui le mettait trop à mal. Deux autres ont adopté des positions défensives rendant inexploitables les réponses. C'est le cas de Moustapha qui a commencé par raconter que tout allait bien, comme s'il récitait une leçon apprise, avant de reconnaître, ou plutôt de faire comprendre, les yeux cachés derrière les mains, que c'était difficile pour lui. 
" - As-tu des personnes importantes ici sur qui tu peux compter?

Non.

- C'est un peu difficile?

(Il acquiesce de la tête).

- C'est pour cela que tu n'as pas envie de raconter?

(II fait comprendre que oui).

- Et avec les jeunes de la maison?

Ça se passe bien.

- Ce sont des copains ou juste des colocataires?

Des colocataires.

- A Bordeaux, tu te sens à l'aise ou c'est encore un peu trop nouveau?

Nouveau.

- C'est un peu difficile pour toi ici?

Ouais. "

Moustapha, malien, 16 ans.

Deux jeunes se sont montrés quant à eux très revendicatifs, subissant les contraintes conjoncturelles puisqu'après avoir été déplacés dans le cadre de la péréquation, leur orientation tardait à venir et leur accueil provisoire s'éternisait.

"Je suis dans cette association depuis un peu longtemps. Les choses ne bougent pas trop selon moi. Ils sont un peu trop lents par rapport aux démarches. Cela n'avance pas trop. Quand les jeunes demandent à avoir un métier, c'est lent. Les éducateurs ne font pas assez d'efforts. Je suis un peu déçu. Toujours attendre. Tout le monde a le même avis que moi. Ça ne va pas. Cela ne marche pas bien. Ils n'essaient pas de nous comprendre. Ils s'en fichent. Ils ont leur salaire. " Lamin, ivoirien, 16 ans.

Les autres se sentent à l'aise dans les services. Ils s'adaptent et apprécient le soutien dont ils bénéficient. Des liens se tissent.

"J'aime le groupe. Si j'étais isolé, je ne serais pas heureux. Je suis habitué à cela. En Afrique, la famille est très grande. A l'école aussi, j'étais toujours avec mes amis. Au foot... J'ai grandi avec la mentalité de la société. [...] Avec les autres jeunes, c'est facile, nous avons quitté la même souffrance." "

Chilemba, guinéen, 16 ans.

"Moi, ça me donne beaucoup d'expérience de voir des choses différentes. Les éducateurs, les éducatrices, les chefs, me donnent des choses différentes. Ils me disent beaucoup de choses et j'ai vu beaucoup de choses que je ne voyais pas chez nous. Ici, on t'apprend comment faire seul. Je trouve que c'est très important dans la vie d'un être humain!" Idrissa, guinéen, 14 ans. 
Pour résumer, en phase d'évaluation, l'inactivité et l'attente sont douloureuses pour ces jeunes qui se sentent dans l'urgence. Le suivi enclenché, outre le besoin de voir les projets d'insertion se mettre en place, une incompréhension des exigences de l'environnement est facteur de mal-être et les difficultés d'intégration prennent une place importante dans le vécu. Constat qui peut être mis en lien avec la pyramide des besoins de Maslow, l'appartenance et la socialisation venant juste après les nécessités physiologiques et de sécurité.

\subsection{Une fois installé, reconquérir ses libertés}

Au fil du parcours au sein de l'Aide Sociale à l'Enfance, l'étayage apporté est mis en balance avec le caractère contraignant de la prise en charge. Au début, les règles sont peu contestées. On les trouve " normales ", voire protectrices. C'est le cas d'Asani qui juge l'heure de retour le soir adaptée, précisant avoir " peur » de sortir seul la nuit. Certains peuvent se dire surpris ou déstabilisés par les fonctionnements institutionnels, mais ils ne portent pas de jugement négatif et ils tentent de s'adapter.

"Il y a beaucoup de règles! Comme de manger le matin, le midi et le soir. C'est ça qui me pose un peu problème. Chez nous, on mange quand on a faim! Ici, tu manges à l'heure! A 12h00, il peut arriver que je n'aie pas mangé et là, tu es obligé de manger! Mon ventre lui, il est habitué à manger vers 15 h00 ou 16h00, comme quand je rentrais de l'école en Guinée! Mais je n'ai pas fin à midi pile! Chez moi, chacun mangeait quand il avait faim. On pouvait manger ensemble mais pas forcément. A part ça, il y a beaucoup de règles ici qui sont comme chez nous ! " Idrissa, guinéen, 14 ans.

Habiter seul en appartement à son arrivée peut d'ailleurs mettre en difficulté. La plupart des jeunes ont toujours vécu entourés et outre la gestion de la solitude, ils n'ont pas eu à assumer les différentes tâches du quotidien. L'accueil en foyer ou en semi-collectif peut alors s'avérer rassurant et plus adapté.

"Les deux premiers mois, c'était dur pour moi! Je me sentais seul et ma mère me manquait. Elle me manquait beaucoup. Je leur ai dit que je ne pouvais pas rester à I'hôtel, seul. C'était dur de m'occuper de moi-même. De me lever, d'aller à l'école seul, de me préparer à manger. Des fois, je n'avais pas le moral pour cuisiner parce que je n'étais pas habitué alors j'achetais des kebabs en ville avant de rentrer à la maison. En Guinée, moi je n'arrivais jamais à me préparer, à être autonome et à faire des choses seul. Je n'arrivais pas à me lever, j'attendais toujours que quelqu'un vienne me réveiller pour aller à l'école. Là-bas, ce sont les mamans qui réveillent, ce sont les mamans! Et je ne cuisinais jamais pour moi ! » Idrissa, Guinéen, 14 ans. 
Cependant, ceux que l'on peut considérer comme " installés " (accueillis depuis plus d'un an) sont plus critiques face aux foyers. Seuls Anton et Léa mettent en avant un souvenir positif de leurs expériences du collectif. Le groupe a constitué pour eux une source précieuse d'étayage et leur a permis d'apprendre de l'Autre. Léa apprécie toutefois aujourd'hui d'être davantage responsabilisée dans le service de chambres en ville.

"Là-bas, au foyer, c'était comme une famille! J'ai beaucoup aimé ! Je me sentais bien car j'étais entourée d'adultes. Je me sentais en sécurité. Cela me rassurait. Le groupe, ce n'était pas facile, il y avait des hauts et des bas, mais il ne fallait pas rester bloqué sur les choses banales. C'est comme au travail! Cela m'a beaucoup appris! [...] Dans ce service, c'est au top car ils font confiance, ils ne sont pas derrière toi. Je me sens à l'aise dans l'appartement et j'aime avoir des responsabilités. Quand j'ai des doutes, j'envoie des messages à mon éducatrice et on fait ensemble. "

Léa, congolaise, 21 ans.

Les autres jeunes insistent sur les contraintes liées aux tâches collectives et aux règles de fonctionnement en foyer. Un mineur explique qu'il aimerait être "comme tout le monde " et pouvoir "souffler" le soir après les cours, en retrouvant ses copains. Régulièrement dans les propos ressort l'opposition entre les libertés octroyées dans le pays d'origine et les règles institutionnelles.

"C'était différent, j'étais libre là-bas, chez moi! Mais ici, au foyer, je n'étais pas libre, c'était fermé pour moi. "

Hamid, afghan, 18 ans.

"Au foyer, les règles n'étaient pas difficiles à comprendre. Mais un peu quand même. Par exemple, au foyer, il y avait des horaires pour rentrer le soir. Au Mali, après manger, la nuit, tu vas où tu veux mais à 7 h00 ou 8 h00 du matin, tu dois être rentré à la maison pour travailler. "

Oussy, malien, 18 ans.

"Moi, j'aimerais avoir 25 ans en fait! 30 ans comme ça! Profiter de pouvoir tout faire tout seul! Là où je suis, par exemple au foyer, je n'aimerais pas y être toute la journée. II $y$ a des gens qui te disent de faire ça ou de ne pas faire ça. Si je pouvais choisir une vie, un âge, ce ne serait pas 15 ou 16 ans. Ça ne serait pas ça. C'est comme une prison, moi je considère cela comme une prison! Il y a des règles. En Afrique, il n'y a pas ça. Même si tu vis avec tes parents, tu sors à l'heure que tu veux, tu rentres à l'heure que tu veux, même si tu as 5 ou 6 ans. Tu as grandi comme ça. Quand on arrive ici, on nous dit de ne pas sortir après 22h00. Et chaque jour à l'école. C'est difficile! Si j'avais choisi un âge ici, je n'aurais pas pris 15 ou 16 ans. "

Sekou, malien, 16 ans.

Ces plaintes se rapprochent de celles de tout adolescent réclamant plus de libertés. Avec une dimension culturelle en plus puisque ces jeunes peuvent comparer avec les fonctionnements qu'ils connaissaient dans leurs pays et bien souvent là-bas, 
tant qu'ils remplissaient leurs obligations, ils étaient libres de sortir comme bon leur semblait. Sans parler de la traversée durant laquelle ils ont pu être livrés à eux-mêmes. Sauf qu'ici, le statut de mineur s'accompagne de certaines restrictions. Plus encore peut-être dans les services de protection de l'enfance où, comme ironisent souvent les jeunes, tout projet de sortie fait l'objet d'une concertation d'équipe, voire d'une demande d'autorisation à l'inspecteur. "Ce que j'ai compris " nous dit Sékou, " c'est que quand on est petit ici, on n'a pas beaucoup de pouvoir ". Les jeunes trouvent d'ailleurs souvent paradoxales les nombreuses règles qui s'appliquent aux mineurs, pour laisser place à tous les droits à l'âge de 18 ans.

"Ici, quand on est majeur, on peut décider, participer dans la maison, on peut signer des feuilles sans les parents... Ils font ce qu'ils veulent, les parents ne peuvent rien dire parce qu'ils sont majeurs. Ils sortent quand ils veulent, rentrent quand ils veulent. Alors que lorsqu'ils avaient 17 ans, ils avaient des règles. C'est bizarre pour moi. »

Farhad, afghan, 17 ans.

Les services de l'Aide Sociale à l'Enfance appliquent des règles dans une visée protectrice. Mais cette notion ne prend pas forcément sens pour les Mineurs Non Accompagnés. Hamina peut dire qu'avoir des éducateurs qui "veillent sur eux", comme leur a expliqué le chef, lui semble important. « Oui, j'ai besoin qu'on s'occupe de moi " ajoute-t-elle. Mais pour beaucoup, la notion de protection n'évoque pas grand-chose. Là-bas, il n'y a pas de droits spécifiques pour les mineurs. Pour Baakir, ce rôle revient exclusivement aux parents. "Protéger, ça dépend! Si tu es avec ta mère oui. A côté de ta maman, de ton père. "Demande de soutien et demande de protection ne sont pas synonymes. La tension est visible ici entre le besoin d'étayage d'un côté et la difficulté à accepter les contraintes afférentes de l'autre, entre l'aspiration à l'autonomie et la dépendance générée par le contexte nouveau et le statut de mineur.

Le soutien apporté dans les services permet cependant d'en supporter les exigences. En effet, malgré le caractère contraignant des fonctionnements institutionnels et plus spécifiquement des foyers, les douze jeunes interrogés (installés ou sortis du dispositif) mettent en avant l'étayage dont ils bénéficient. Être aidé dans les démarches administratives est le premier élément qui ressort.

" J'ai galéré pour avoir le titre de séjour, ils me demandaient le passeport. Il y a mon éducateur qui m'a beaucoup aidé. Il y a des gens qui se débrouillent bien, qui n'ont pas besoin d'aide, mais moi non, j'ai besoin d'aide. Sur les papiers, sur le travail, sur tout quoi!»

Walid, afghan, 18 ans. 
"Les éducateurs, moi ça m'apporte beaucoup parce que d'abord je ne connais pas trop les lois ici et ça m'apporte plein de choses, des connaissances au niveau des droits. J'apprends avec eux donc quand je vais être tout seul, autonome quoi, je vais essayer de faire tout seul parce qu'on m'aura montré. "

Semei, ivoirien, 18 ans.

Le soutien moral, mais aussi le fait de pouvoir parler et d'être compris, sont également valorisés.

"S'il n'y avait pas le service à côté de moi, s'il n'y avait pas des gens qui sont à côté de moi pour essayer de me parler, pour me dire des mots, ça aurait été chaud. A un moment donné, moi, j'ai pensé directement... Surtout le mois dernier après la police aux frontières... Il y avait un truc dans ma tête qui était de me suicider pour laisser le monde. Pour laisser le monde directement, oui. Ça, c'est arrivé dans ma tête. Cette idée, je ne vous mens pas, franchement. Je pensais à ça tout le temps. Je me disais ça va quoi, ça suffit." "

Issan, guinéen, 18 ans.

"La chef de service, quand je la vois, ce n'est pas ma maman mais je la vois comme une maman. Elle peut me dire des choses, d'arrêter ça... Elle est gentille. Je respecte tout le monde mais c'est à elle que je dis les choses. Je l'écoute plus. C'est elle qui m'a fait comprendre beaucoup de choses. Quand elle me parle, je comprends vite. "

Sekou, malien, 16 ans.

En résumé, à leur arrivée, les Mineurs Non Accompagnés sont demandeurs d'une prise en charge contenante et les foyers ou semi-collectifs répondent à leurs besoins. Au fil de leurs parcours, une fois les démarches d'insertion entreprises et le processus d'adaptation à la société d'accueil enclenché, les règlements sont perçus comme contraignants et les jeunes aspirent à davantage d'autonomie. Ils disposent d'un certain recul sur les services et s'autorisent à adopter un regard critique. Même si le soutien reçu compense les contraintes, leurs besoins ne sont plus les mêmes et proposer des modalités de prise en charge évolutives paraît essentiel.

\section{EXIGENCES DE RÉGULARISATION ET D’AUTONOMISATION}

\subsection{De la méconnaissance du droit au séjour à la conscientisation des enjeux}

La question du droit au séjour est abordée au cours de tous les entretiens. II s'agit de mesurer d'une part le niveau d'information, en lien avec l'âge et l'étape à laquelle se trouve chacun dans le parcours à l'Aide Sociale à l'Enfance, d'autre part l'inquiétude que peut générer ou non l'exigence de régularisation.

Six jeunes n'ont pas d'informations précises sur la demande de titre de séjour. Huit autres sont informés des procédures, au moins partiellement, mais déclarent ne pas y penser. Leurs priorités sont ailleurs (la scolarisation par exemple) et ils s'évitent 
des préoccupations qui n'ont pas encore lieu d'être. Ces quatorze jeunes se trouvent en phase d'évaluation, de recours, ou en début de prise en charge.

"Cela m'inquiète, mais pas trop en fait. Je ne veux pas me prendre la tête pour rien, alors que cela ne va pas encore arriver $"$.

Sekou, malien, 16 ans.

"Les choses qui me préoccupent, c'est d'aller à l'école et de jouer au foot. Mais sinon, il n'y a rien qui m'inquiète!"

Idrissa, guinéen, 14 ans.

Sept jeunes, aux différentes étapes de la prise en charge, se disent sereins quant à la régularisation. Ils ne perçoivent pas forcément d'obstacles à l'obtention du titre de séjour et certains pensent avoir un rôle à jouer, indiquant que la décision dépendra de leur bon comportement ou de leur motivation. Attribuer la régularisation au fait de payer ses factures (propos énoncés à plusieurs reprises) laisse entrevoir une connaissance erronée du droit au séjour. Si la marge de manœuvre existe effectivement dans l'investissement scolaire et professionnel, les critères administratifs sur lesquels les jeunes ont peu de prise sont ici mis de côté, comme pour garder la maîtrise de leur avenir.

"Le titre de séjour, cela vient de moi-même. Si je suis les règles d'ici, ça ira. Cela dépend de ma volonté, il ne faut pas faire de bêtises ".

Saïd, ivoirien, 16 ans.

"Le titre de séjour, s'ils le donnent c'est bien! Sinon, tant pis! Mais ce serait con de ne pas me le donner alors que je m'intègre et que j'étudie. Seule la personne en elle-même peut être obstacle à ses projets! ".

Léa, congolaise, 20 ans.

Douze jeunes témoignent au contraire de l'inquiétude générée par les démarches de régularisation. Les appréhensions portent (ou portaient) principalement sur la possible contestation des documents administratifs, pouvant conduire à des situations incongrues du fait de niveaux d'exigences différents selon les pays. Des obstacles peuvent aussi se présenter dans les démarches consulaires, comme nous le soulignions précédemment. Le cas de la Guinée qui ne délivre pas de passeports actuellement génère inquiétude et colère à l'égard des autorités du pays.

Lorsque les dossiers ont été déposés, les jeunes appréhendent une réponse qui ne dépend plus d'eux. Ils ne savent pas "à quoi s'attendre " et "ce qu'ils vont décider ». Leur avenir est entre les mains des administrations. Ils peuvent en perdre le contrôle et ils le savent, d'où les préoccupations, voire parfois les angoisses. Par 
contre, une fois régularisés, ils se disent rassurés et peuvent se projeter, au niveau professionnel surtout.

"Au niveau de la préfecture, c'était plus fort que moi. J'ai tellement entendu le mot attendre, attendre, attendre... A un moment donné, je n'arrivais pas à dormir et je me disais qu'il fallait que je me rende là-bas, qu'ils m'avaient peut-être oublié et qu'il fallait que je me rende là-bas.»

Issan, guinéen, 18 ans.

" J'étais tout le temps inquiet jusqu'à ce que j'aie mes papiers! Je ne savais même pas si j'avais le droit de rester en France. Et si j'allais avoir les papiers ! C'était très compliqué ! Ce n'était pas facile! Je ne voulais pas retourner dans mon pays. Je savais comment était la vie là-bas par rapport à ici ! »

Anton, kosovar, 25 ans.

Pour ceux qui rencontrent des obstacles administratifs, le vécu est tout autre. Les équipes éducatives le confirmeront, ces situations se multiplient. En effet, la rétention des documents d'état civil par la préfecture pour vérification d'authenticité se fait dorénavant quasi systématique, prenant parfois de nombreux mois ; la légalisation au consulat est devenue obligatoire pour certaines nationalités et le récépissé n'est plus délivré en cas de "faille " dans le dossier. Des mises en examen pour usage de faux sont également prononcées. Par ailleurs, les employeurs n'étant pas toujours au fait des démarches, les autorisations de travail tardent à arriver. Pendant ce temps, les jeunes se trouvent freinés, voire bloqués dans leurs parcours d'insertion. Issan explique comment les difficultés de régularisation ont entraîné jusqu'à une baisse d'investissement dans sa formation.

Chaque chose en son temps! Les Mineurs Non Accompagnés se renseignent progressivement sur les conditions de droit au séjour mais pour la plupart, ne s'y intéressent qu'à l'approche de la majorité, comme s'il s'agissait à chaque étape de répondre aux exigences de l'instant pour apporter une nouvelle pierre à l'édifice du parcours d'installation. A mesure que l'échéance approche et que s'enclenchent les démarches de régularisation, les informations se font plus précises et ils tendent à perdre le sentiment de maîtrise de la situation. Si la délivrance d'un titre de séjour permet de se stabiliser et de se projeter, les obstacles administratifs ferment quant à eux progressivement les portes.

\subsection{Une insertion socio-professionnelle par étapes}

Les attentes des jeunes en matière d'insertion évoluent au fil de leurs parcours à l'Aide Sociale à l'Enfance et de leur installation dans la société d'accueil, en lien avec leur âge et l'approche de la majorité. Commençant très souvent par des cours de 
Français Langue Étrangère, ils sont ensuite scolarisés en collège ou en lycée, avant d'enclencher, pour la plupart, une formation professionnelle en alternance.

Les douze jeunes rencontrés en phase de recours ou en évaluation bénéficient de cours de français au sein d'associations. Ils souhaitent aller à l'école, apprendre un métier ou travailler. La majorité a déjà des idées de profession (cuisinier, footballeur, plombier, boulanger, militaire, agriculteur, pompier, policier, carrossier, électricien, conducteur de camions, ingénieur en construction, conducteur de bateaux etc.)

L'amélioration du niveau de langue est une priorité pour une grande partie et les cours dispensés répondent ainsi à leurs attentes. Une minorité de jeunes regrette de ne pas être scolarisée.

"Je veux d'abord apprendre le français. Après, on verra. Je veux aussi me faire des amis, jouer et sortir avec eux. Le français est une barrière. Quand je parlerai, ce sera bon. " Hamidi, algérien, 16 ans.

"On m'envoie pour suivre les cours, comme ça. Moi, j'ai dépassé le niveau! Je ne peux même pas me concentrer parce que cela ne sert à rien! Des fois, on nous prend pour des gens qui ne connaissent rien! Des fois, on nous considère comme des bêtes qui ne connaissent rien! ”

Osei, guinéen, 16 ans.

Osei juge paradoxal de devoir réfléchir à des idées de métiers sachant que dès qu'il fait des propositions, on lui oppose maints obstacles.

«-Ambulancier?

Personne ne va payer le permis pour toi.

- Aide-soignant?

Non, non, non, il faut que tu passes le bac pro, c'est impossible !

- Pompier?

Non, tu n'as pas la nationalité française.

- Policier?

Non plus, il faut encore la nationalité française.

- A 18 ans, je travaillerai, je paierai la formation que je veux, ce sera facile ! ”

Osei, guinéen, 16 ans.

Parmi les jeunes pris en charge dans les services éducatifs, six âgés de 14 à 16 ans sont scolarisés au collège et un septième, de 17 ans, au lycée. La plupart sont en classe d'intégration. Tous ont un projet d'orientation. Certains évoquent des difficultés scolaires. Plus de la moitié dit ne pas se sentir encore intégrée, mettant en avant les difficultés de communication, le fait d'être parmi les rares étrangers dans un établissement situé en milieu rural et enfin, l'absence de contacts avec des élèves 
français en classe d'intégration. Mais ils s'arment de patience et se montrent confiants pour la suite.

"Au collège, j'aimerais apprendre beaucoup, mais comme je n'ai jamais été à l'école... Je n'ai jamais utilisé le téléphone au pays. Quand on me dit de faire des choses avec l'ordinateur, c'est impossible ! C'est difficile! En anglais et en espagnol, je ne peux pas. Je connais quatre ou cinq langues dans mon pays. Là, j'arrive à apprendre le français. Je me débrouille. Mais l'anglais et l'espagnol, en plus, je ne peux pas ! [...] Comme moi, on est quatre. Tu te trouves tout seul, Noir, au milieu de huit-cents élèves français ! C'est dur ! Mais on va y arriver quand même. C'est dur mais on va y arriver. " Sekou, malien, 16 ans.

"Moi, je suis rentré pour parler bien français. C'est important! Quand je comprendrai bien le français, je chercherai beaucoup de choses! Comme le travail, les copains, les copines... Si tu commences le travail et que tu ne parles pas français, ce n'est pas bon! Si le patron te parle et que tu ne comprends pas, c'est un problème! J'écris bien, je lis bien, mais parler, c'est difficile pour moi !"

Asani, malien, 16 ans.

Le jeune scolarisé au lycée met en avant les difficultés rencontrées dans la recherche d'employeur, accentuées selon lui par le fait d'être étranger. Il évoque aussi l'exigence de réussite.

"Depuis le mois de novembre, j'enchaîne des stages. Parce que je voudrais faire de la plomberie. Mais c'était très difficile de trouver l'apprentissage! Vu l'accent d'abord, quand tu appelles, l'autre ne te comprend pas. Un patron m'a même dit qu'il ne me prendrait pas parce qu'il avait eu un jeune qui ne savait ni lire ni écrire. Je lui ai répondu de ne pas considérer tous les jeunes de la même manière. Et lui, comment il a appris ? Ce n'était pas difficile au début? Qu'il ne me sous-estime pas! J'ai appelé plus de deuxcents entreprises! C'était difficile ! [...] Je n'ai pas le droit à l'erreur, je dois faire tout pour bien comprendre ce qu'on me dit. Je n'ai pas envie de les décevoir. Travailler bien, ne pas avoir de mauvaises notes. Ce n'est pas bon, on m'a dit ça. II faut toujours bien travailler et essayer de faire le maximum. "

Ange, guinéen, 17 ans.

Mis à part ce lycéen en pôle d'accompagnement, les neuf autres jeunes pris en charge depuis plus d'un an à l'Aide Sociale à l'Enfance sont en alternance. Huit passent un $\mathrm{CAP}^{40}$ (électricité, boulangerie, cuisine, boucherie et horticulture), dont cinq qui envisagent de poursuivre les études vers un $\mathrm{BP}^{41}$ ou un baccalauréat, voire un $\mathrm{BTS}^{42}$. Une jeune effectue un titre professionnel en vente, en parallèle à la passation d'un baccalauréat en candidat libre.

\footnotetext{
${ }^{40}$ Certificat d'Aptitude Professionnelle.

${ }^{41}$ Brevet Professionnel.

${ }^{42}$ Brevet de Technicien Supérieur.
} 
"J'aimerais aller le plus loin possible. Plus tard, c'est sûr que je travaille et si cela se passe bien, je suis le chef et je gère un chantier. "

Farhad, afghan, 17 ans.

Lorsque nous rencontrons les jeunes, les formations ne sont pas terminées et leur avenir au sein des entreprises n'a pas forcément été discuté mais plusieurs indiquent être appréciés dans leur travail et se sentir bien intégrés.

"L'électricité, ça me plaît, ça me plaît trop! Il y a beaucoup de travail, tu n'as pas de temps libre! Avec l'équipe, cela se passe bien. Les collègues s'occupent de moi. Il faut car je suis apprenti! Au lycée aussi! C'est pour cela que je suis content ici, il n'y a aucun problème ! Je suis étranger, et alors, il n'y a rien du tout ! "

Walid, afghan, 18 ans.

" $\mathrm{Au} C \mathrm{CFA}^{43}$, ça va. Tous les professeurs, ils m'aiment. Et les copains, c'est pareil! Présentement, je suis en train de m'intégrer parce que je suis en formation, je travaille, j'approfondis mon français. Pour pouvoir vivre comme tout le reste des jeunes. ״

François, camerounais, 18 ans.

Enfin, les deux personnes qui ont quitté le dispositif sont en emploi dans le secteur du bâtiment, un en intérim et le second en contrat à durée indéterminée.

Si l'on reprend ces parcours scolaires, tous les jeunes rencontrés en début de prise en charge ont l'intention de se former et d'apprendre un métier, dont une grande majorité avec déjà des idées de secteurs professionnels. Les cours de français répondent dans la plupart des cas à une nécessité immédiate, même si certains regrettent de ne pas être scolarisés plus tôt. Ce désir d'aller à l'école, assez présent à l'arrivée en France, laisse progressivement place à un projet de formation professionnelle en alternance, répondant au besoin d'émancipation financière. Malgré parfois des difficultés scolaires, les jeunes interrogés présentent des compétences appréciées tant dans les établissements qu'en entreprise. La valorisation de leurs parcours leur permet de se projeter dans une poursuite d'études.

\subsection{Une injonction à l'autonomie pressante}

«Pour le public des jeunes sortant des dispositifs de protection de l'enfance, le passage à la vie autonome s'avère rapide et brutal. Ces jeunes doivent faire face à toutes les transitions en même temps plutôt que graduellement (Stein et Munro, 2008) alors même qu'ils y sont peu préparés et qu'ils disposent de moins de support et de compétences sociales que les jeunes de la population générale (Goyette 2007). » (Dans Oui, 2012). Les transitions évoquées sont celles vécues par tout adolescent

\footnotetext{
${ }^{43}$ Centre de Formation d'Apprentis.
} 
entrant dans l'âge adulte. Nous retiendrons le passage de l'école au travail, de I'hébergement familial (ici institutionnel) au logement autonome et enfin, de la dépendance à l'autonomie financière. Cependant, les jeunes pris en charge à l'Aide Sociale à l'Enfance sont soumis à un délai pour franchir ces étapes, qui répond à un critère administratif $d$ 'âge. Les contrats jeunes majeurs permettent de reculer l'échéance. Créés lors de l'abaissement de la majorité à 18 ans en 1974, ils offrent aux départements la possibilité de maintenir la protection et l'assistance éducative jusqu'à 21 ans. II s'agit de dispenser un accompagnement à ces jeunes ayant atteint le statut de "majeurs", mais pas forcément autonomes dans toutes les sphères de la vie quotidienne.

Selon Vincenzo Cicchelli, l'expression "jeunes adultes" relie deux termes a priori « contradictoires» pour signifier «l'allongement de la dépendance entre les parents et les jeunes » (2001). L'auteur compare l'évolution des modes d'émancipation des jeunes. Alors qu'auparavant, l'autonomie passait par la décohabitation, c'est-àdire " une séparation spatiale entre les générations ", dans la société contemporaine, elle se construit « dans le cadre d'un parcours individuel de maturation psychique et sociale qui légitime le rôle de médiation des parents" (Scabini et Cigoli, 2007). Ces derniers facilitent en effet les démarches d'insertion de leur enfant, mais aussi sa construction en tant qu'individu "autonome ". II continue d'appartenir à sa famille tout en redéfinissant " les modalités d'attachement à ses parents " et en poursuivant sa socialisation. Pour les jeunes placés à l'Aide Sociale à l'Enfance, ces rôles de médiation et d'insertion sont justement assurés par les services éducatifs. L'autonomisation est, comme au sein d'une famille, matérielle, mais aussi psychique. " II faut travailler la séparation » entend-on dans le jargon des éducateurs, autrement dit, favoriser l'investissement $d^{\prime}$ 'une relation éducative et permettre ensuite aux jeunes de s'en détacher progressivement pour voler de leurs propres ailes.

Selon la situation et l'âge auquel a lieu l'admission dans le service, le temps de suivi peut suffire ou non. D'autant que dans le cas des Mineurs Non Accompagnés, les difficultés sociales auxquelles Anne Oui (2012) fait référence (problèmes d'insertion, de logement, de santé, fragilités psychologiques etc.), peuvent être renforcées du fait de leurs parcours, mais aussi de leur statut spécifique en France. Pourtant, l'injonction à l'autonomie est d'autant plus forte pour ce public. En effet, au regard du nombre croissant de demandes de prises en charge dans certains départements et du manque de places, les durées d'accompagnement tendent à se réduire. 
Les jeunes interrogés ont des réactions très différentes au sujet de l'accès à l'autonomie selon l'étape à laquelle ils se trouvent dans leurs parcours au sein de I'Aide Sociale à l'Enfance. Neuf en phase de recours, d'évaluation ou en début de prise en charge ne se projettent pas encore dans l'avenir. Leurs priorités sont ailleurs. A mesure que les situations évoluent et que l'installation s'opère, la question se pose de façon plus concrète. En plus des deux jeunes ayant quitté le dispositif qui vivent dans leur propre appartement, quatorze aspirent à leur autonomie. Ils attendent que les conditions soient réunies pour pouvoir accéder à leur propre logement et « se débrouiller » seuls. Huit autres ne se sentent pas encore prêts et évoquent leurs inquiétudes. Les propos de Semei, tout juste majeur, illustrent ce mélange d'émotions. II met en avant son appréhension face aux démarches administratives et à la gestion financière, mais aussi face à la solitude.

"Cela fait peur! Être autonome, les factures, les meubles que tu dois acheter... Si tu n'es pas prêt financièrement, ça va être un peu chaud quoi! C'est comme si tu rentrais dans la vraie vie, dans la vie des gens, d'un travailleur qui travaille! Pourtant, moi je suis apprenti ! Cela me fait un peu peur! Apprenti et tu fais ça! [...] Et puis j'ai un peu peur parce que j'ai toujours vécu avec des gens et tout ça mais... pour le moment je ne suis pas encore à cent pour cent prêt mais je pense que ça ira. Gérer tout ça... Cela fait peur! [...] Oui, ils ont trop d'attentes! Déjà, ils me disent que vite, je dois quitter la structure. Mais d'abord, l'homme doit se construire! Tout petit, tu es avec ta maman, tes frères et sœurs. Tu sais que petit à petit, en grandissant, tu vas quitter ta famille. C'est comme ça. Mais quand tu n'es pas encore prêt et que ta famille te dit de quitter la maison, tu fais comment? "

Semei, ivoirien, 18 ans.

Pour Semei, la sortie du dispositif est précipitée et il fait le parallèle avec un enfant à qui sa famille demanderait de partir, trop tôt. La question du statut est ici centrale. Un adolescent n'est pas encore un adulte. Nous développerons dans la troisième partie les concepts liés à la jeunesse et à l'ambivalence de statut. Semei nous dit aussi qu'un apprenti n'est pas un « travailleur », dans le sens où il l'entend lui, c'està-dire un salarié gagnant un salaire complet. On lui demande pourtant d'être autonome financièrement. Pour lui, les exigences à son égard sont trop élevées. N'estce pas ce que tentent de nous dire nombre de jeunes à travers ce que les équipes nomment les « revendications financières » ? Ange l'explique très bien. II ne comprend pas que le foyer lui demande une participation financière. "Je n'ai pas 18 ans, je n'ai pas un salaire qui avoisine par exemple les cinq-cents euros ". Pour un jeune de 19 ans qui s'apprête à quitter le dispositif, il peut l'entendre, mais dans son cas, à 17 ans, il trouve cela un peu tôt. Anne Oui reprend le constat de la Cour des Comptes de 2009 : " le passage d'une logique de protection pour un mineur à une logique d'insertion et de responsabilisation de jeune adulte est toujours difficile » (2012). 
Enfin, Semei évoque un élément culturel d'importance, à savoir que dans certaines sociétés, on n'est pas habitué à vivre seul. Oussy l'exprime aussi, en quelques mots: «Nous, on ne prend pas d'apparts!" Nombreux sont les jeunes interrogés qui expliquent que chez eux, même marié, on reste vivre avec sa famille. Souvent, on agrandit la maison pour fonder son propre foyer, à côté des siens. Ils précisent que le niveau social peut influer sur les pratiques puisque si l'on en a les moyens, on peut effectivement s'installer dans un logement indépendant. Mais ce n'est pas la majorité des cas. Nous constatons néanmoins que les Mineurs Non Accompagnés s'adaptent à la société d'accueil, comme en témoigne leur capacité à se projeter dans ce nouveau mode de vie individuel.

"Moi, ce que je veux, c'est être autonome, avoir mon espace, ma chambre personnelle, pouvoir gérer mon espace. On m'a demandé : "Dans ton pays, c'est comme ça que tu étais ? Tu dormais seul dans une chambre? " J'ai dit que ce n'était pas l'affaire de mon pays. Mon pays, c'est mon pays! Ici c'est ici! [...] Quand j'aurai mon appartement, je vais essayer de m'adapter. Cela va être difficile oui ! Mais après, ça va aller. Au fil du temps, tu changes, les choses changent pour toi. Du côté positif, j'espère! Je serai comme, par exemple, vous ! Moi, je vais faire comme ça après ! Cela me plaît ! »

Ange, guinéen, 17 ans.

Les équipes, conscientes des fragilités et des besoins potentiels de ces jeunes, envisagent (comme pour les autres) la mise en place de relais auprès d'institutions de droit commun en prévision de leur sortie. Cependant, lors des entretiens, il n'y est pas allusion. Comme si toute possibilité d'étayage était absente en dehors de la prise en charge. II semble que la question du lien soit à interroger pour ces publics qui, après un parcours migratoire souvent traumatique et de nombreuses ruptures, se sont autorisés à s'appuyer sur des services et n'ont pas forcément l'énergie pour investir de nouveaux lieux ressources plus à distance. Entre le foyer avec une présence éducative quotidienne et le service de chambres en ville proposant des rencontres hebdomadaires, l'écart est conséquent et souvent déstabilisant, mais les jeunes s'adaptent et ils y trouvent généralement leur compte. Mais en quittant le dispositif, passer la porte du $\mathrm{CCAS}^{44}$, expliquer de nouveau sa situation pour s'entendre dire que I'on peut rencontrer l'assistante sociale de permanence, mais uniquement sur rendezvous entre $9 \mathrm{~h} 00$ et $17 \mathrm{~h} 00$, pour des démarches très ciblées, avec un délai d'attente de cinq ans pour les demandes de logement, c'est un cap que tous ne sont pas forcément en mesure de franchir. Une réflexion est menée dans certains départements sur l'accompagnement des plus de 21 ans et la mise en place de structures intermédiaires paraît en effet appropriée pour ce public.

\footnotetext{
${ }^{44}$ Centre Communal d'Action Sociale.
} 
Une injonction forte à l'autonomie donc, pour les jeunes pris en charge dans les services de l'Aide Sociale à l'Enfance, d'autant plus difficile à vivre peut-être pour les Mineurs Non Accompagnés sous certains aspects. Nous constatons néanmoins combien ils sont capables de s'adapter non seulement à la société d'accueil, mais aussi aux exigences institutionnelles. II aurait été intéressant de rencontrer davantage de jeunes ayant quitté l'Aide Sociale à l'Enfance pour recueillir le vécu de cette étape de transition et de leurs parcours post-institutionnels.

\section{CONCLUSION DE LA DEUXIÈME PARTIE}

Les Mineurs Non accompagnés se confrontent dans la société d'accueil à des exigences élevées, souvent en décalage avec les pratiques rencontrées dans les pays d'origine. Les lois en matière de droit au séjour peuvent constituer un frein au processus d'installation. A l'inverse, le dispositif de protection de l'enfance, par son caractère exigent, favorise la concrétisation des projets en tendant vers l'autonomisation. II se constitue en intermédiaire et en vecteur d'adaptation. Notre étude met en évidence combien les besoins et les possibilités des jeunes évoluent au fil de leur installation, d'où l'importance de leur proposer des prises en charge individualisées qui intègrent la notion de parcours. 


\section{PARTIE 3 : UNE JEUNESSE DANS L'ENTRE-DEUX}

\section{ENTRE DEUX ÂGES, ENTRE DEUX CULTURES}

\subsection{L'âge des incohérences statutaires}

Pierre Bourdieu, dans son entretien avec Anne-Marie Métailié en 1978, propose une définition de la jeunesse. II la considère non pas comme " une unité sociale " rapportée à "un âge défini biologiquement " mais comme une séquence de trajectoire biographique. La "trajectoire sociale » étant entendue par le sociologue comme " la série de positions successivement occupées par un même agent (ou un même groupe) dans un espace lui-même en devenir et soumis à d'incessantes transformations " (dans Mauger, 2001). La jeunesse est donc une étape dans le parcours de vie et sa spécificité est le caractère "temporaire " de son statut : " mienfant, mi-adulte ", "ni enfant, ni adulte ". Ici encore, il est question de statut et plus précisément $d$ '« incohérence statutaire ». Le sociologue parle d'un " no man's land social » dans lequel les jeunes sont " adultes pour certaines choses », " enfants pour d'autres ».

Les Mineurs Non Accompagnés ne sont-ils pas considérés comme trop petits pour vivre seuls, trop petits pour rentrer à l'heure qu'ils veulent le soir, trop petits pour signer leurs documents administratifs, puis rapidement trop grands pour rester dans les services de protection de l'enfance? A la fois trop fragiles aux yeux de la société pour ne pas être encadrés et brutalement trop mâtures pour l'être. Leurs propos sont également paradoxaux. "Je sais faire ", " je gère tout seul " entend-on çà et là, mais aussi, comme nous le signifiions précédemment, " je ne suis qu'apprenti, je n'ai pas encore 18 ans, j'ai encore besoin d'être aidé ! " Être pris en charge, être porté, tout en jouissant de certaines libertés. On retrouve ici toute l'ambivalence de I'adolescent. Là où elle est peut-être la plus parlante, c'est lorsqu'un jeune ayant quitté le dispositif rappelle son ancien éducateur, plus qu'il ne le faisait en étant dans le service, lui demandant par exemple conseil sur la gestion du budget, alors que quelconque regard sur ses comptes lui était auparavant insupportable. C'est une fois la contrainte de la mesure levée qu'il sollicite du soutien, dégagé du statut d'enfant et des limites associées. Ni enfant, ni adulte, dans un entre-deux, en construction.

"Je ne suis pas un adulte. Je ne suis pas un enfant non plus. Au Mali, on ne parle pas d'adolescent. " Sekou, malien, 16 ans. 
"Là-bas, à l'âge de 7 ans, si tu es musulman, tu vas commencer à jeûner, à prier, tu ne vas pas faire de choses bizarres... Les parents ont déjà fait leur devoir et toi, tu vas commencer à remplir tes obligations. A l'âge de 7 ans, tu es quand même un enfant, mais pour les parents, dans leur tête, tu n'es plus un enfant. Parce qu'à 7 ans, tu vas commencer à étudier, tu vas commencer à partir à l'école toi seul, tu vas commencer à laver les habits pour toi seul. Tu commences à avoir un peu de responsabilités. » Ouma, guinéen, 17 ans.

"Comment je me considérais à 16 ans ? Je sentais que je grandissais et que les 18 ans arrivaient $"$.

Léa, congolaise, 21 ans.

Cet entre-deux ressort explicitement dans ces témoignages. Le " ni-ni », pour reprendre une formule populaire. Ils ne sont pas encore adultes mais ne sont pas vraiment des enfants puisqu'ils commencent à avoir des responsabilités, ou du moins, à faire des choses comme les adultes. Ces jeunes ne trouvent pourtant pas de mots pour décrire ce statut. Léa, pour sa part, met en avant l'évolution et la transition vers l'âge adulte. Grandir. Apprendre et acquérir progressivement les rôles adultes. "La jeunesse peut être définie sociologiquement comme la phase de préparation à l'exercice de ces rôles adultes, ce que les sociologues appellent la socialisation." (Galland, 1991, p.131). A chaque âge, nous dit Olivier Galland, ou à chaque classe d'âge pourrait-on préciser, "des rôles fonctionnels et des positions " alloués, mais aussi à I'inverse, un apprentissage de ces rôles par un processus de socialisation (Ibid., p.104). Ces rôles, les jeunes interrogés les appellent les « responsabilités ».

Les sociologues de la jeunesse utilisent aussi le concept d' " apesanteur » pour décrire cet état intermédiaire, comme en suspens. "L'apesanteur familiale caractérise cette séquence de trajectoire biographique, dans la mesure où l'affranchissement progressif de la famille d'origine permet de se soustraire, pratiquement et subjectivement, aux contraintes qu'elle exerce, sans pour autant devoir déjà se soumettre à celles d'une nouvelle famille [...]. Les privilèges s'acquièrent progressivement, [...] alors que persistent des interdits de l'enfance. A l'inverse, de nouveaux devoirs apparaissent tandis que disparaissent des privilèges de l'enfance. " (Mauger, 2010). On endosse de nouvelles responsabilités, mais on n'en est pas non plus au stade de fonder une famille.

"Non, moi je ne devais pas chercher d'épouse. Je n'étais pas arrivé à ce stade. Je n'avais pas cet âge-là pour être marié. Même s'il y a des hommes qui se mariaient! Mais moi... Je n'étais pas considéré comme un adulte, non !!! Maman, à chaque fois... Non !!! J'étais très, très loin! Personne ne pouvait me parler de ces trucs-là, jamais! II ne faut pas penser à cela! Se marier? " (rires)

Ange, guinéen, 17 ans. 
"Chez moi, j'étais encore considéré comme un enfant. Je n'étais pas considéré du même niveau que les autres parce que j'étudiais. On me disait juste d'aller à l'école. »

Chilemba, guinéen, 16 ans.

Le fait d'étudier, nous dit Chilemba, préserve des contraintes et des responsabilités qui incombent aux adultes. Ses propos sont peut-être ceux qui traduisent le mieux cette notion d' "apesanteur". "On me disait juste d'aller à l'école ». C'était (et c'est toujours) son rôle. Étudier. Contribuer en retour au prestige de la famille ? Il le vit presque comme un luxe. Il a été " désigné » pour cela, alors que les autres membres de sa famille doivent travailler pour collaborer à l'économie familiale. Cette période de transition, tout le monde n'a pas l'opportunité de la vivre. Elle n'existait pas non plus auparavant dans la société française, l'individu passant directement du statut d'enfant à celui d'adulte chargé de trouver un travail et de fonder une famille. "C'est quoi un adolescent ? " demandent certains durant les entretiens. Dans les sociétés modernes, la jeunesse prolonge cette étape intermédiaire et permet de se construire, de se centrer sur soi, sur ses intérêts et ses projets. Pierre Bourdieu préfère au terme de "jeunesse " celui de "jeunesses", en lien avec ces différentes modalités de passage à l'âge adulte, qui sont fonction de la classe sociale. Ce droit à vivre sa jeunesse n'est pas équitable. Certains peuvent plus se permettre de prendre le temps que d'autres. N'est-ce pas ce que tentent de nous dire les jeunes qui, dans le service d'évaluation, scandent leur désir d'aller à l'école, à la vraie école, celle des enfants, comme tout le monde?

"Un apprentissage? Non, je n'ai pas envie de faire ça moi ! Je n'ai pas d'enfants, je ne suis pas marié Madame ! Pourquoi je vais me presser à faire du travail ! Je suis un enfant moi!Il faut me comprendre! Moi, je veux aller à l'école. C'est mon objectif premier! Voilà ! Je veux prendre mon temps ! La manière dont les autres sont libres... La façon dont ils font leur vie, les Français qui sont nés ici... Ils étudient, ils ne font rien... C'est comme ça moi que je veux être ! Je ne veux pas être gêné comme ça! " Osei, guinéen, 16 ans.

Osei dit être un enfant. II veut prendre son temps, comme les autres, vivre tel un jeune, en somme. Il s'insurge contre les exigences institutionnelles pour faire valoir son droit à vivre sa condition de jeune, souvent dérobé aux mineurs de l'Aide Sociale à l'Enfance et encore plus aux migrants pour qui la régularisation constitue un enjeu de taille.

S'affirmer, les Mineurs Non Accompagnés le font en quittant leurs pays. Pour Thomas Fouquet (2007), aujourd'hui, « les jeunes jouissent d'un pouvoir plus important, selon la terminologie de Weber : c'est-à-dire une capacité ou une chance plus grandes pour réaliser leur destin personnel. Un espace plus étendu d'autonomie 
se dessine en effet, parce que la crise des systèmes d'autorités traditionnelles (hérités) suscite un relâchement du contrôle social qui s'exerce sur eux. " C'est ce que nous démontre Oussy qui est passé outre les décisions de son père en prenant la route. On s'émancipe en faisant le choix de la migration, pour ensuite se soumettre à une mesure de protection pour le mineur que l'on est, avec les contraintes que cela implique.

Les Mineurs Non Accompagnés se trouvent dans cet entre-deux statutaire lié à leur âge, "mi-enfants, mi-adultes», avec une dimension culturelle en plus, la condition d'adolescent n'existant pas forcément chez eux. Nous allons voir que la notion d'âge administratif, qui fait frontière entre la minorité et la majorité, déterminant ainsi les droits et les obligations, n'a pas la même signification pour eux.

\section{2. Âge administratif versus âge des responsabilités.}

Nous demandons aux jeunes ce que cela change dans leurs pays d'avoir 18 ans. La réponse est généralement « rien » ou " je ne sais pas, je ne suis pas encore arrivé à cet âge ! " N'a-t-on pas plus de responsabilités ? " Peut-être, oui ! » A quel âge est-on majeur là-bas ? "Comme ici, 18 ans, 20 ans, quelque chose comme ça ! " Doit-on faire de nouvelles choses comme travailler ? "Hein ? Mais à 7 ans, 5 ans on peut travailler déjà ! " Les échanges sont peu fluides. Les questions paraissent incongrues. Cette référence à l'âge, pourtant si présente dans notre société, n'a pas la même signification là-bas.

"La majorité, cela existe mais pas trop, pas comme ici ! Tu es un enfant jusqu'à 5 ans, 6 ans, après ça s'arrête là. Tu te débrouilles. II y a des enfants qui travaillent depuis leurs 3 ans. Ce ne sont pas des adultes mais ils travaillent, ils font la même chose que les adultes. " Sekou, malien, 16 ans.

" Ha bah avec le travail au noir, il n'y a pas d'adultes et pas d'enfants ! II n'y a rien ! Tu travailles seulement!» Hamid, afghan, 18 ans.

Dans les sociétés d'origine, c'est le mariage qui permet d'accéder au statut $d^{\prime}$ adulte (nous le détaillerons en suivant). Il n'est donc pas question de majorité, d'âge administratif mais d'âge de responsabilités. Prendre en charge, c'est être adulte. Pour d'autres, on est grand quand on fait des choses de grands, en partant travailler avec son père, par exemple. 
"Chez nous, dès que tu as 10 ou 11 ans, tu es considéré comme un grand! Quand tu commences les trucs avec ton père, à 11 ans comme ça, à 16 ans, tu es grand! La majorité commence dès que tu es avec ton père et qu'il t'explique des choses. Là-bas, on n'attend pas que tu aies 18 ans. On ne peut pas donner d'âge exact. "

Oussy, Malien, 18 ans.

Agnès Roche, dans son article intitulé « Les jeunesses au prisme de la sociologie. État des lieux " (2006), rappelle qu'Émile Durkheim considérait la fonction plus importante que l'âge. Les jeunes n'étaient pas considérés par le sociologue comme un groupe particulier susceptible de susciter un intérêt d'étude.

Pour Idrissa, l'expérience prime sur l'âge. Propos qui rejoignent ceux de Sékou pour qui la maturité est associée au savoir.

"Ici, on étudie en âge. Chez nous, on étudie à l'expérience. Là-bas, si tu n'arrives pas à gagner les notes suffisantes pour passer à la classe supérieure, tu restes dans la même classe."

Idrissa, guinéen, 14 ans.

"J'ai des copains qui sont plus jeunes mais qui connaissent plus de choses que moi. Ils sont plus matures. Comme une copine, qui a 12 ans et qui me dit ce que je ne dois pas faire. Elle est toute petite mais c'est elle qui me donne des conseils. Alors qu'elle n'a que 12 ans! »

Sekou, malien, 16 ans.

L'âge n'a-t-il pas d'importance là-bas ? Oussy nous explique que non, l'âge ne compte pas pour lui. II ne s'intéresse pas à cette donnée quand il rencontre quelqu'un, l'essentiel est que ce soit une "bonne personne ". II trouve étrange que les enfants clament à qui veut l'entendre qu'ils sont grands alors qu'ils viennent de souffler leurs trois bougies! Oussy n'a d'ailleurs jamais fêté son anniversaire.

"Au Mali, il n'y a pas cela, l'âge. Quand j'étais petit, je ne connaissais pas le mien. Chez nous, quand tu ne sais pas ton âge, tu demandes à ton père et c'est lui qui va dire à quelle date tu es né. "

Oussy, malien, 18 ans.

Gérard Mauger, dans son article "Jeunesse : essai de construction d'objet " (2010), indique que l'âge présente deux acceptions différentes. II peut être entendu comme "la durée écoulée depuis la naissance " (Larousse) ou comme " une période de la vie allant approximativement de tel âge à tel autre " (Robert). Cette seconde définition renvoie à la notion de "cycle de vie ", "séquence ordonnée d'étapes familiales, scolaires, professionnelles " et à " une série de positions " associées. C'est cette dernière acception qui semble primer dans les pays d'origine des jeunes. La mesure du temps importe peu. Ce qui prend sens, c'est la place occupée au sein de la 
famille, la fonction et le rôle que l'on joue. Olivier Galland parle de normes d'âges conditionnant les rôles sociaux. "L'accès aux positions sociales et la reconnaissance du statut de citoyen sont ordonnés sur l'échelle des âges. " (1991, p.103). Selon lui, l'échelle est plus sociale que biologique.

Nous l'avons vu, dans de nombreuses sociétés, être adulte signifie avoir la capacité de contribuer à la solidarité familiale. Être petit, ce n'est pas forcément avoir moins de 18 ans mais être dépendant. A son arrivée dans le pays de destination, dans cet inconnu, se déclarer mineur, c'est signifier son incapacité à s'assumer seul et à endosser un rôle de responsable. Lorsque l'on "prend un autre âge ", on écarte ce critère administratif insignifiant au profit de la notion de besoin. La minorité est associée à la possibilité de soutien, mais aussi à l'accès aux droits, comme celui d'aller à l'école.

"C'est ici que j'ai connu les droits. Là-bas, il n'y a pas de droits ! Si on te dit d'arrêter l'école et que tu ne veux pas, si tes parents te comprennent, tu continues tes études, mais sinon, ils vont te maltraiter. Ce sont tes parents qui décident à ta place. " Vinza, ivoirien, 16 ans.

L'âge administratif, qui détermine dans notre société le statut de mineur ou de majeur, ne prend pas forcément sens dans les sociétés d'origine où c'est la prise de responsabilités qui confère un statut. Se déclarer mineur, plus qu'une référence à l'âge, traduit un état de dépendance et un besoin d'étayage.

\subsection{Devenir adulte là-bas et ici}

Pierre Bourdieu relevait en 1980 trois critères d'entrée dans la vie adulte renvoyant à des changements de statut: l'accès au travail, le départ de la famille d'origine et le mariage (Galland, 1991, p.55). S'ils peuvent paraître d'une certaine banalité, il est pourtant fort intéressant de se pencher sur ces paramètres et de les confronter à une approche spatio-temporelle. Qu'en est-il dans les pays d'origine ? Et dans la société d'accueil, aujourd'hui, quarante ans plus tard ? Les écarts sont nets.

Lorsque l'on demande aux jeunes à quel âge on est considéré comme un adulte dans leurs pays, la réponse est la suivante: "Quand on se marie ». Ils précisent cependant que se marier ne signifie pas quitter sa famille, ou du moins pas pour les garçons qui font généralement venir leurs épouses. Le mariage, donc, est un critère de passage à l'âge adulte. Le départ de la famille d'origine pas forcément. L'accès à l'emploi non plus, puisque la plupart travaillent depuis qu'ils sont petits. En effet, le travail n'a pas d'âge. 
"En Guinée, si tu es marié, tu es un adulte ! C'est toi qui te réveilles ! Les mamans n'ont plus besoin de te réveiller!"

Idrissa, guinéen, 14 ans.

Dans certaines familles, on explique aux jeunes vers 16 ou 17 ans qu'ils sont dorénavant adultes et doivent s'assumer. Dans d'autres, père et mère n'attribuent pas les mêmes rôles à leurs enfants.

"Maintenant, tu es grand, tu dois être fier de toi, tu peux trouver un travail, tu peux finir tes études, tu peux te débrouiller tout seul. "

Farhad, afghan, 18 ans.

"Mon père me considérait comme un grand et ma mère comme un petit. Mon père me disait que j'étais grand maintenant, qu'il fallait que je travaille, mais ma mère non. Elle voulait que j'apprenne et avait demandé à mon oncle de m'enseigner un peu l'écriture. " Walid, afghan, 17 ans.

Cécile Van de Velde (2007) présente quatre modèles d'entrée dans la vie adulte en Europe, mettant en lien les trajectoires des jeunes avec les contextes économiques et culturels. La jeunesse " longue " danoise favorisant le développement personnel est très éloignée du vécu des Mineurs Non Accompagnés. Le passage à l'âge adulte fondé sur le mariage, comme le décrit ce public, se rapproche en revanche du modèle espagnol dans lequel "l'expérience de la jeunesse est caractérisée par l'attente au foyer parental des conditions nécessaires à une installation stable dans la vie adulte. [...] Elle s'inscrit [...] dans des normes culturelles valorisant l'appartenance familiale et conditionnant le départ à la création d'un nouveau foyer [...]. Majoritairement associées à l'idée de la stabilité économique et aux responsabilités familiales, les représentations que les jeunes espagnols développent de l'âge adulte sont soustendues par cette logique de l'installation. " (Van de Velde, 2007, pp.28-29). Une nuance est à apporter cependant concernant le public de notre étude pour qui le mariage fait effectivement devenir adulte mais n'implique pas un départ du domicile parental. On assiste à une séparation symbolique des générations qui ne se traduit nullement au niveau des espaces de vie contrairement au cas des Espagnols.

En France, nous dit Cécile Van de Velde, les jeunes expérimentent une forme de "semi-dépendance ", la famille venant en soutien financier durant la période des études afin de faciliter la construction du "futur statut social de l'individu ". "L'âge adulte est envisagé comme l'âge de la stabilité définitive ". "Être mûr ", "être responsable " et " se sentir autonome " sont les caractéristiques de l'adulte (Ibid., pp.27). Si l'on revient aux critères énoncés par Pierre Bourdieu, le mariage est mis de côté au profit du travail et du logement individuel. Cette conception impacte 
directement les exigences à l'égard des Mineurs Non Accompagnés qui s'installent en France. Devenir adulte, c'est se stabiliser au niveau de l'emploi et financièrement. Nous pourrions donc penser qu'ils sont amenés à passer de leur modèle (proche du schéma espagnol, nous l'avons dit), à celui de la société d'accueil. Pourtant, pour ces jeunes, la dimension temporelle n'est pas la même que pour les Français. Certes, notre public vit une phase de "semi-dépendance ", que l'on peut attribuer au temps de prise en charge au sein de l'Aide Sociale à l'Enfance. Ils ont en effet quitté le domicile familial mais sont soutenus par le dispositif, dans un entre-deux. Cependant, cette étape intervient bien plus tôt que pour les Français, entre 16 et 18 ans dirons-nous, alors qu'elle commence en général bien après la majorité pour les autres. A 18 ans, les Mineurs Non Accompagnés s'apprêtent à quitter ce dispositif de "semi-autonomie " pour endosser les rôles adultes.

Ce modèle est celui du Royaume-Uni, où la société « favorise le développement $d^{\prime}$ 'une forme de jeunesse plus courte, orientée vers un accès rapide au statut social et familial d'adulte. L'individu est invité à s'assumer de la façon la plus précoce possible » (Van de Velde, 2007, p.25). Le recours aux prêts étudiants est fréquent et encouragé par l'État. Si les Mineurs Non Accompagnés en France ne bénéficient pas de cette facilité, le soutien financier accordé le temps de leur prise en charge leur permet de se constituer une épargne en vue de leur sortie. Au Royaume-Uni, «la prise d'indépendance résidentielle est précoce ", vers 21 ans. "Le départ constitue une réelle rupture symbolique dans les trajectoires et dans les relations intergénérationnelles. La norme sociale invite l'individu à devenir adulte, c'est-à-dire un être de responsabilité pourvoyant à ses propres besoins. " (Ibid., p.25). Notons que cette rupture symbolique, notre public la vit une première fois avec la famille, puis une seconde avec les équipes éducatives qui l'accompagnent quotidiennement le temps de la prise en charge.

Ce schéma rappelle celui que suivaient en France les jeunes "ouvriers", en opposition aux « bourgeois ». Dans son entretien avec Anne-Marie Métailier en 1978, Pierre Bourdieu évoque un "brouillage de ces oppositions" du fait de l'accès généralisé à l'enseignement secondaire qui retarde pour tous l'entrée dans le monde du travail. Néanmoins, elles ont bel et bien existé, comme en témoigne l'étude de Laurence Wylie citée précédemment. En ville, "l'âge de 21 ans correspond sans doute à la période où l'on se sent et où l'on agit en adulte ", alors qu'à Peyrane, on se marie vers 18 ans (Wylie, 1968, p.152). Dans ce village, pourtant, les rôles de l'adolescent sont bien distincts de celui de l'adulte. " Un adolescent ne doit pas se conduire comme un adulte. » (Wylie, 1968, p.131). II profite de libertés et est incité à sortir, recevant 
son pécule. Même s'il travaille, il ne dispose pas de son argent propre puisqu'il remet les bénéfices aux parents, contribuant ainsi à l'économie familiale sans encore jouer un rôle d'adulte. Ces pratiques rappellent grandement celles décrites par notre public en Afrique. "Quand les jeunes mariés reviennent à Peyrane pour s'installer dans le logement qu'on leur a aménagé, ils se rendent compte que leur statut a changé. Pour eux, l'adolescence est terminée. Ils sont mari et femme maintenant, c'est-à-dire des adultes qui devront désormais assumer leurs responsabilités devant la vie. " (Ibid., p.163). Il est donc question ici de statuts et de rôles. Quand on est jeune, on s'amuse, on contribue à l'économie familiale mais on n'a pas le statut de responsable. Se marier et prendre en charge une famille confère le statut d'adulte.

Précisons que le voyage migratoire est une autre façon de devenir adulte: «transition vers un meilleur avenir et quasi rite de passage vers le statut d'aîné social ", nous dit Thomas Fouquet (2007). Contribuer à l'économie familiale, permettre d'accroître les ressources, mais aussi le "capital symbolique " en apportant du prestige social, c'est grandir. "Schématiquement, devenir adulte en Occident renvoie largement au fait de parvenir à subvenir à ses propres besoins en entrant dans la vie active (s'opposant ainsi à la passivité supposée du jeune), de quitter le domicile des parents pour éventuellement fonder à son tour un foyer ou une famille, etc. L'échec ou le renoncement à cette prise d'autonomie précoce sera perçu négativement. Au Sénégal - et plus globalement dans de nombreuses sociétés du Sud, la donne semble encore bien différente: le "grand" (c'est-à-dire le détenteur de l'autorité sur, notamment, son propre devenir) est celui qui trouve les moyens et ressources de prendre sa part positive au système de la solidarité communautaire. " (Ibid.). En endossant ce nouveau rôle, on change sa position au sein de la famille. Les rôles sont inversés. C'est ce que Thomas Fouquet nomme la restauration du «transfert intergénérationnel » (2007). On ne compte plus sur ses parents, ce sont eux qui attendent de nous un soutien. On est responsable.

« Partir, c'est chercher une forme de complétude tout en essayant de changer le regard que l'entourage porte sur nous - et par conséquence la place que l'on occupe au sein de la société d'origine. [...] Plus concrètement, le voyage migratoire représente une transition possible entre la jeunesse et l'âge adulte." (Ibid.). C'est ce que Maamuni a compris en entendant les mots de sa mère à son départ : " Si tu sors, si tu pars là-bas, tu n'auras pas papa, ni maman, donc là-bas, tu vas te débrouiller toi seul. II te faudra des papiers et ce sera difficile. " Farhad aussi s'est senti adulte en prenant le chemin de la migration. 
"Depuis que j'ai quitté mes parents, je me sens un adulte, moi. Je fais ma vie tout seul. Quand j'ai quitté, j'avais 14 ans et je me suis dit dans ma tête qu'il fallait penser que j'étais un adulte et que je pouvais tout faire tout seul parce que je n'avais plus mes parents et qu'il fallait que je me débrouille tout seul. A partir de cela, je me suis dit que j'étais un adulte. "

Farhad, afghan, 17 ans.

La migration, transition donc entre deux espaces, mais aussi entre deux périodes de la vie. Il y a le proche et le lointain, tout comme il y a l'avant et l'après migration. Thomas Fouquet décrit le voyage comme un « espace-temps intermédiaire, permettant de faire le lien entre deux moments de l'existence distincts " (2007). De la même façon, la jeunesse conduit de l'enfance à l'âge adulte, la finalité étant la construction d'une place dans la société. Une place d'étranger disposé à s'intégrer dans le pays d'accueil. Grandir dans un ailleurs. Pour ce faire, les Mineurs Non Accompagnés mobilisent les ressources à leur disposition.

\section{DES PROJETS ET DES ACTEURS}

\subsection{Différentes logiques d'action}

Julien Bricaud met en avant le rôle actif des usagers dans l'orientation des interventions sociales qui leur sont dirigées. Ils ne sont pas juste bénéficiaires, ils contribuent dans l'interaction à faire évoluer les dispositifs. "Politiques des services sociaux et attentes des usagers se combinent pour dessiner les circuits d'aide et d'assistance. " (2012, p.80). Le public des Mineurs Non Accompagnés illustre parfaitement ces propos. Nous l'avons signifié en introduction, il bouscule les pratiques et interroge les dispositifs. A la frontière entre deux âges, mais aussi entre la protection de l'enfance et le droit des étrangers. On ne sait pas toujours comment appréhender ces jeunes ni quelle intervention mener auprès d'eux. C'est dans les interactions qu'ils guident les équipes, laissant entrevoir, derrière certaines résistances parfois, leurs capacités, mais aussi leurs fragilités. "Pris dans les entrelacs de la politique sociale, les usagers sont actifs, ils prennent certaines directions, en repoussent d'autres, $s^{\prime} y$ perdent ou $s^{\prime} y$ retrouvent, participent à leur propre définition. " (Ibid., p.81).

Pour faire concorder leurs attentes avec les exigences des dispositifs, il est essentiel de comprendre ce qui les anime, ce qui guide leurs actions, leur façon de voir le monde et de s'y mouvoir. Nous allons pour cela recourir à la théorie de l'expérience développée par François Dubet comme grille de lecture. Elle met en évidence les trois logiques d'action que peut adopter, à plus ou moins grande échelle, tout individu. Dans le registre de l'intégration, il se définit par ses appartenances. Dans celui de la 
stratégie, il vise la réalisation de ses intérêts. Enfin, la subjectivation désigne le développement du sujet critique face à la société de domination (1994, p.111). Appréhender l'acteur selon cette approche permet de cerner la façon dont il perçoit le monde qui l'entoure et comment il interagit avec son environnement. Parler de stratégie concernant le public des Mineurs Non Accompagnés peut avoir une connotation péjorative vu les doutes pouvant exister sur la véracité de leurs récits de vie. Cependant, d'un point de vue sociologique, ce terme renvoie à la capacité d'agir d'un individu et de faire des choix réfléchis. Il est considéré acteur de son parcours, en mesure de mobiliser les ressources à sa disposition pour réaliser ses intérêts dans une société concurrentielle. Les Mineurs Non Accompagnés ont quitté leurs pays et leurs familles, affronté la dure réalité du trajet migratoire, pour se confronter ensuite à une société d'accueil méconnue et exigeante dans laquelle le processus d'adaptation prend du temps. Ils le font dans un désir d'émancipation, dans une quête de statut pour eux mais aussi pour leurs familles. Ils sont déterminés à réaliser ce pour quoi ils sont partis, ne renoncent pas et ne repartent pas tant qu'ils n'ont pas atteint leurs objectifs. Lorsque les institutions opposent à leurs projets des contraintes, ils réajustent et s'adaptent. Ils se mobilisent autour de leur scolarité et manifestent leur incompréhension face au dilettantisme de certains de leurs camarades pour qui les enjeux ne sont pas les mêmes. Ils se font leur place sur le marché du travail, dans la société, pour réussir. Ils savent ce qu'ils veulent.

"Il y a des patrons qui préfèrent prendre des étrangers. Souvent. Tu sais pourquoi ? Parce que nous on est plus travailleurs. Ça c'est sûr ! Parce que nous on est plus motivés que celui qui est là, qui a maman à la maison, qui rentre, qui jette ses chaussettes partout. Parce qu'on a la volonté. Parce qu'on en veut. Parce qu'on sait ce qu'on fait. On sait où on va aller. On sait ce qu'il y a derrière. "

Redei, camerounais, 21 ans.

Le registre de la stratégie est souvent très présent chez eux. Le positionnement de Redei en est une illustration, presque caricaturale. Le vocabulaire qu'il utilise fait référence au calcul et à l'anticipation, à la survie aussi : "j'ai observé », " je savais vers qui me tourner en fonction des situations ", "j'ai donné l'âge de 16 ans ", " ce qui m’a niqué ", " on a des filières partout ", " dans la vie, il faut savoir sauver sa peau ", "j'ai appris à calculer », " t'es baisé », etc.

"Pour les titres de séjour, on m'avait dit que c'était un chemin. Un chemin comme on dit ! Qu'il faut faire un truc pour avoir ça. II faut arriver mineur pour avoir les papiers... Etre sur le chemin. Ou il faut se marier ! (rires) Il faut avoir des gamins! Tu sais comment ça marche!" Redei, camerounais, 21 ans. 
Pour Redei, c'est le contexte qui pousse à " calculer » : " Si on arrivait en France et que ce n'était pas si dur d'avoir les papiers... " Le temps est compté. "Chaque jour qui passe, c'est un jour en moins, et c'est la majorité qui approche $»$. II met en avant la nécessité d'être bien conseillé, d'avoir « le bon éducateur ».

"Quand l'éducatrice ramène le dossier à la préfecture pour le présenter, elle est sûre qu'il n'y a pas de failles dedans, elle est sûre que ça va marcher, parce qu'elle l'a étudié, parce que ce n'est pas juste aujourd'hui qu'elle le fait, parce que cela fait sept-cents dossiers déjà qu'elle a ramenés. Mais si déjà elle a oublié la photocopie, qu'elle a oublié qu'il ne fallait pas présenter ça... Elle fait mal son boulot. On est d'accord ou pas ? On fait tous mal notre boulot mais il y a des choses qu'il ne faut pas faire mal parce qu'à la préfecture, quand tu ramènes un dossier, les gens, au moindre coup d'œil, ils peuvent te dire non. Et c'est un non qui te détruit toute ta vie."

Redei, camerounais, 21 ans.

Cette façon d'agir, Max Weber la nomme comportement « rationnel en finalités », en opposition aux comportements "traditionnels», "affectuels » et "rationnels en valeurs" (1922, p.55). Il le définit comme "des expectations du comportement des objets du monde extérieur ou de celui d'autres hommes, en exploitant ces expectations comme "conditions" ou comme "moyens » pour parvenir rationnellement aux fins propres, mûrement réfléchies, qu'on veut atteindre » (Ibid., p.55).

Un autre registre est aussi fortement activé chez les Mineurs Non Accompagnés, celui de l'intégration. Notre étude a révélé que le sentiment de ne pas être encore intégré était source de mal-être chez de nombreux jeunes se trouvant en début de prise en charge. Ne pas avoir encore de copains, se sentir à la marge de la société ou encore discriminé, les met à mal. Ils cherchent à être intégrés par les pairs. Améliorer le niveau de français et atténuer l'accent est pour certains une condition avant de s'aventurer dans la rencontre. Ils aspirent à être et à vivre comme " eux ", souhaitant se définir par cette nouvelle appartenance. Ce qui n'empêche pas de garder un lien fort avec les siens, de revendiquer ses origines, dans une double appartenance. "Je suis bordelais d'origine congolaise ", nous dit un jeune. Un autre affiche son tee-shirt du Cameroun. Le week-end, on achète le riz à l'épicerie africaine et on partage un mafé avec les copains. Le "Nous » ne vient pas tant en opposition au « Eux » dont on cherche à s'approcher et à qui l'on souhaite parfois ressembler, mais il préserve son identité. "Chez nous ", " là-bas ", " au bled ", renvoient à ce registre de l'intégration, au même titre que « je suis bordelais ». 
Tout en évitant de généraliser, chaque situation étant unique, la subjectivation, posture que l'on peut supposer davantage politisée, visant à contester l'ordre social établi et la domination, est probablement la logique d'action la moins manifeste chez ce public. Pourtant, nous le verrons, un processus d'individuation s'opère et au fil de leur parcours à l'Aide Sociale à l'Enfance, les jeunes tendent à s'affirmer. Ils peuvent s'opposer aux institutions, comme c'est le cas pour les questions d'ordre financier, mais aussi matériel ou de commodités, lorsqu'ils expriment, par exemple, le sentiment de ne pas être considérés face à un logement qui ne correspond pas à leurs attentes.

François Dubet (1994) nous dit que le bien-être d'un individu est atteint lorsqu'il parvient à un équilibre entre ces trois registres de l'action. Réussir son insertion professionnelle permet d'accéder à une certaine stabilité certes, mais si l'on est isolé, sans ses attaches, se sent-on épanoui? Nous l'avons dit, les Mineurs Non Accompagnés, le temps de la prise en charge, tiennent. Ils endurent et tiennent. Ils visent la réalisation de leurs objectifs. Ils sont généralement entourés, pratiquent le sport, s'adaptent à leur environnement. Mais il n'est pas rare, face à un obstacle, comme le refus d'un patron de prolonger un contrat, ou des complications dans leurs dossiers administratifs, de les voir s'effondrer et se renfermer sur eux, coupant les liens avec leurs amis, mais aussi la famille qu'il ne faut pas inquiéter. Et dans la durée, une fois leurs objectifs d'insertion atteints, comment se vivent-ils dans la société d'accueil ? Comment ressentent-ils l'éloignement de leurs familles et de leurs pays, une fois passée la période d'adaptation et une fois l'installation durable ?

Les Mineurs Non Accompagnés, donc, privilégient les logiques d'intégration et stratégique. Pour s'insérer et ainsi réaliser les intérêts individuels et familiaux, différents moyens peuvent en effet être mobilisés. La sollicitation d'un accompagnement éducatif en est un, tout comme le fait de se faire reconnaître mineur.

\subsection{L'âge qu'il faut}

Comme nous l'expliquions précédemment, les choix de destination peuvent naître de renseignements reçus en amont du projet migratoire ou durant la traversée. Une fois sur place, chacun fait appel aux ressources mises à sa disposition. Dans le cas des Mineurs Non Accompagnés, le réseau familial étant absent, ils se voient contraints de développer de nouvelles stratégies individuelles ou collectives pour pouvoir s'installer. Faire valoir ses droits en s'adressant aux services du Conseil Départemental en est une. Nuancer son récit pour avoir plus de chances d'être pris en charge en est 
une autre, dictée par les passeurs ou les jeunes qui se trouvaient « devant » durant la traversée.

"Ici, quand tu ne mens pas, on ne te prend pas! II faut que tu mentes! Quand tu dis Ia vérité, on ne te prend pas! Ils sont comme ça! C'est ça le problème! C'est les passeurs qui nous disent ça! II faut être fort ! Moi, je fais le malin ! "

Osei, guinéen, 16 ans.

Nous l'avons dit, la société d'accueil est exigeante, fondée sur des critères administratifs souvent éloignés de la culture d'origine. Chacun se débrouille alors comme il peut. Michel de Certeau utilise le terme de " braconnage " pour décrire " les façons dont chacun déjoue les attentes, s'arrange avec les lois et les conventions, interprète les codes existants pour les combiner avec ses propres intérêts". Selon lui, "les individus développent une créativité dispersée faite de ruses et de détournements pour se réapproprier les comportements qui leur sont imposés par la norme " (dans Bricaud, 2012, p.83). On me demande d'être mineur pour être pris en charge, je me déclare mineur.

Contourner la norme est une stratégie fréquente. Comment se maintient une telle organisation et que font les jeunes de la parole entre eux ? Cette identité "stratégique » est-elle celle que l'on donne aux institutions uniquement ou aux pairs également ? Certains disent ne pas poser de questions aux copains, ne pas parler de cela. Il arrive cependant à untel de trouver sur les réseaux sociaux des photos de tel autre entouré de sa femme et de ses enfants au pays. Mais il semble que la pratique soit au silence. Comme si c'était la règle. Comme si le " monde migratoire " avec ses codes se prolongeait au-delà de la traversée, dans la phase d'installation. Dire que l'on ne connaît personne dans le foyer est une méthode dont a usé Redei. Pourquoi ? "C'est comme ça, c'est la politique ! » Des fois que l'on transmette le concernant des éléments qu'il n'a pas donnés. Cette politique préserve les intérêts de tous. Ce n'est qu'après avoir quitté le dispositif que l'on peut s'autoriser (ou pas) à en parler.

Une de nos hypothèses était que les stratégies opérées par les Mineurs Non Accompagnés pour réaliser leurs projets pouvaient menacer leur identité intégratrice. Comme celle de modifier son âge. Comment un individu peut-il se situer dans son histoire s'il doit en gommer une partie ? Quel impact aura sur son développement l'inclusion au sein de groupes de jeunes dont l'âge ne lui correspond pas? L'introduction de la notion de "classe d'âge " répond à ces interrogations. Comme l'explique Olivier Galland, certaines sociétés régies par le principe générationnel fonctionnent par groupes d'âges, qui franchissent les grades aux mêmes moments, les 
différentes étapes de la vie dictant l'accès aux statuts, aux droits et aux obligations (Galland, 1991, p.65). L'âge en soi n'a pas d'importance (hormis si l'écart est trop important, nuance une psychologue de l'association Mana). La stratégie qui consiste à se déclarer plus jeune répond à un besoin de soutien, sur la base d'un problème (l'âge) qui n'en est pas un. C'est ce que nous dit Redei pour qui l'âge n'a pas d'importance, et il n'est pas le seul. D’autres jeunes évoquent néanmoins de possibles difficultés d'intégration.

"C'est un choix dans la vie! Je m'en fous moi! Chez nous, on ne parle pas d'âge. C'est quoi la différence ? Cela change quoi ? C'est quoi la différence entre quelqu'un qui a 16 ans et quelqu'un qui a 20 ans? On s'en fout de l'âge ! Tu peux avoir 30 ans et réagir comme quelqu'un de 20 ans ! C'est ça hein!"

Redei, camerounais, 21 ans.

"Je connais un Camerounais qui avait 19 ans quand il est arrivé en France mais il a dit qu'il en avait 17. On l'a accepté et on l'a envoyé dans un foyer. Là-bas, il n'a pas pu tenir. Parce que la façon dont il vivait là-bas, en fait il était déjà passé ça. Directement, il est parti pour dire qu'il n'était pas mineur et il a demandé l'asile. "

François, camerounais, 18 ans.

Oussy exprime les deux positions possibles: "Peut-être que c'est difficile mais il y en a peut-être qui s'en foutent ». Opposition ou conciliation ? Ne s'agit-il pas du prix à payer pour être soutenu ? Nous sommes ici dans un rapport entre l'effort et le bénéfice.

"Tu n'as pas le choix! Les règles du foyer, tu es obligé de les supporter! Tu joues le jeu! Tu sais dans quel jeu tu te mets!»

Redei, camerounais, 21 ans.

Redei ironise sur la fausse identité qu'il donne sur les sites de rencontre. Il choisit un autre prénom pour ne pas être reconnu, un âge variable selon qu'il souhaite rencontrer des femmes "plus grandes" ou "plus petites ". "l change son identité, donc, au gré des attentes et des objectifs. N'est-ce pas une pratique moderne chez les jeunes usagers des réseaux sociaux ? Au risque de s'y perdre ? " Mon identité, elle est là ! ", s'exclame Redei, nous signalant son corps et sa tête.

Nous pouvons en conclure que contrairement à notre idée de départ, hormis dans le cas d'un décalage trop important, se déclarer mineur lorsque l'on est majeur pour bénéficier d'un accompagnement éducatif affecte peu le sujet dans une culture où l'âge administratif n'a nullement d'importance. Nous allons maintenant nous pencher sur la question de la filiation. 


\subsection{Nature des liens avec la famille}

Déclarer un autre âge est une chose. Mais indiquer avoir perdu ses parents lorsque l'on a encore sa mère, qu'est-ce que cela produit ?

"Cela m'a fait beaucoup de choses... Dur... Dur de faire des trucs... Dur de... Mais on reste un homme, on reste un battant, on reste un chevalier!"

Redei, camerounais, 21 ans.

Osei, quant à lui, a opté pour une stratégie dans l'entre-deux. Indiquer être séparé de ses parents, sans pour autant dire qu'ils étaient morts.

" D'autres disent que tu peux déclarer ton père décédé et tout ça... Moi, je ne peux pas dire que mon père est décédé ! Pourquoi ? Cela n'a pas de sens! Mon père, il est vivant ! Moi, je ne peux pas dire que mon père est décédé ! Jamais de la vie! Ils disent qu'ils vont avoir pitié de nous si l'on dit ça. Mais moi, je n'ai jamais dit ça! Moi, mes parents ils vivent! Si tu ne veux pas accepter cela, tu me laisses !"

Osei, guinéen, 16 ans.

II précise que déclarer ses parents décédés peut par la suite entraîner des difficultés, au moment de se faire envoyer son acte de naissance, par exemple. " C'est eux qui mentent, ce n'est pas le dossier qui ment ! "Osei pense que quatre-vingt-dixneuf pour cent des jeunes déclarent ne pas vivre avec les parents alors que c'est " absolument faux». Et qu'en est-il de ceux qui sont amenés à emprunter une autre identité, un autre nom que le leur?

"Moi, dans la vie, je ne vais jamais changer mon nom! Quel que soit le problème, mon nom, c'est mon nom! Je vais assumer cela! Quand tu changes ton nom, tout ce que tu as derrière ton nom, c'est foutu en l'air ! Tout ! ”

Oseil, guinéen, 16 ans.

L'association Mana le confirme. L'âge n'a pas d'importance dans certaines sociétés. En revanche, il est essentiel, pour la construction de ces futurs jeunes adultes, de maintenir le lien avec la famille et le pays. "Tuer ses parents " empêche la reconstruction. Pourtant, l'interprétation des lois en matière de droit au séjour peut prêter à confusion et les jeunes intériorisent les restrictions. L'article L.313-11 $2^{\circ}$ bis du CESEDA $^{45}$ stipule qu'un mineur isolé de nationalité étrangère ayant été pris en charge à l'Aide Sociale à l'Enfance avant l'âge de 16 ans peut bénéficier à sa majorité de plein droit d'un titre de séjour mention Vie Privée et Familiale, " sous réserve du caractère réel et sérieux du suivi de la formation, de la nature de ses liens avec la famille restée dans le pays d'origine et de l'avis de la structure d'accueil sur l'insertion de cet étranger dans la société française. " De quelle nature doivent être les liens avec la famille ? Cette condition n'est nullement spécifiée. Dans les débuts de prise en charge

\footnotetext{
${ }^{45}$ Code de l'Entrée et du Séjour des Etrangers et du Droit d'Asile.
} 
des Mineurs Isolés Etrangers, il était question de rupture totale de lien. Que cette interprétation soit fondée ou non, elle était véhiculée et l'est toujours aussi bien dans le "monde migratoire" qu'au sein des équipes professionnelles. Sur le site du ministère de l'Intérieur, on trouve quelques précisions: l'étranger mineur doit " justifier être dépourvu d'attaches dans son pays d'origine ou justifier l'absence de maintien de liens avec sa famille restée dans son pays". Mais là encore, les interprétations de la loi peuvent être trompeuses. Les juristes spécialisés en droit des étrangers explicitent cette clause : le mineur peut dire être en contact avec sa famille mais doit démontrer l'impossibilité d'y être accueilli et pris en charge. Une nuance bancale face à laquelle de nombreux jeunes préfèrent taire les liens plutôt que de risquer de se voir refuser la prise en charge au sein de l'Aide Sociale à l'Enfance et / ou le titre de séjour. Il est essentiel que les équipes soient au fait de la loi et que le tabou soit levé pour que les liens avec les familles puissent être travaillés davantage dans les services éducatifs.

L'identité intégratrice renvoie à la filiation. Être séparé physiquement de la famille par la migration et symboliquement par l'impossibilité de dire les liens constitue une césure dans cette filiation, dans la continuité de son existence. "Les ruptures biographiques, celles de l'adolescence ou de l'immigration par exemple, mettent au jour la crise des filiations engendrant des traumatismes si violents que les individus ne peuvent naître à la nouvelle société s'ils ont perdu les liens à ces racines ». (Dubet, 1994, p.114). Se situer par rapport aux siens, se sentir inclus dans la communauté d'origine et dire ses appartenances, sont essentiels pour pouvoir investir de nouvelles relations.

Si modifier son âge n'a pas forcément d'impact négatif sur l'individu, se couper des relations à sa famille ou les passer sous silence peut être plus compliqué pour sa construction en tant que sujet. Après avoir évoqué les logiques d'action mobilisées par les Mineurs Non Accompagnés dans la société d'accueil, nous allons voir comment ils y prennent place.

\section{ADAPTATION, INTÉGRATION ET INDIVIDUATION}

\subsection{Des étayages multiples pour s'adapter}

Joël Fronteau associe le contexte post-migratoire au processus d'adaptation à l'environnement. En effet, migrer induit de changer les habitudes acquises: langue, climat, alimentation, argent, travail, école, transports, santé, etc. (dans Legault, 2000, p.3). Cela touche "toutes les dimensions de la vie collective et individuelle ", elles- 
mêmes inter-reliées, " les succès atteints ou les obstacles vécus dans l'une constituant des conditions facilitantes ou inhibitrices dans d'autres. "Se confrontent alors "ses pratiques, normes, valeurs de sa culture, avec celles du pays hôte " (Fronteau dans Legault, 2000, p.30). La migration touche jusqu'à l'image de soi, c'est-à-dire à "l'identité tant personnelle que sociale" (ibid., p.3). Elle fait vivre une "crise d'identité ", le sujet craignant " de ne pas voir son identité reconnue » (ibid. p.30), de la perdre au profit de celle de l'accueillant, même si, paradoxalement, il supporte mal d'être traité en " étranger ». " Dorénavant, il faut se résigner à vivre avec deux images de soi, à se voir à la fois comme on se sait être et comme les autres nous voient. A notre identité se trouve superposée, substituée, une nouvelle identité - qui n'en est pas une -, celle de l'étranger. " (Ibid., p.31).

S'adapter, c'est composer avec ces différentes identités, prendre de l'autre, modifier ses façons de percevoir le monde et d'interagir. "Peu à peu, on en vient à expérimenter certains modes de comportement du pays hôte. On entre dans une phase de changement, aussi subtil que profond, qui est aussi une phase de maturation affective. " (Ibid., p.30). Ce changement, c'est celui que décrit Ange lorsqu'il demande à habiter seul plutôt que de partager une chambre en foyer. Vivre en appartement sera difficile pour lui mais il compte "s'adapter ". "Au fil du temps, tu changes, les choses changent pour toi ». Walid aussi décrit à sa manière le processus d'adaptation.

"En Iran ou en Afghanistan, on ne peut pas être en contact avec les filles. Mais ici, quand je suis arrivé, je me suis dit que c'était un truc de ouf ! Au début, c'était bizarre! Elles font la bise! Moi je disais "attends, je n'ai pas l'habitude, ne me fais pas de bisous!" Au collège, je ne voulais pas de bise et les filles comprenaient. Avec le temps, ça passe. Maintenant, c'est normal. J'ai des amies filles. C'est trop, trop facile en plus maintenant. Je ne sais pas pourquoi. Avant, je n'avais pas trop envie, maintenant c'est facile. Si elles veulent discuter, on discute ensemble, on boit un café dehors. En Iran, non, ce n'était pas comme ça non plus. "

Walid, afghan, 18 ans.

"A l'origine de l'adaptation, il y a une rupture ; au cœur de l'adaptation, on trouve l'identité, car c'est un sujet qui se transforme ; et l'objectif de l'adaptation, c'est la recherche de l'équilibre. " (Ibid., p.3). Pour atteindre cet équilibre, le sujet passe par différentes étapes. Joël Fronteau décrit l'adaptation comme « un processus dynamique qui s'inscrit dans le temps, dont la progression n'est pas forcément linéaire et qui se réalise à des rythmes différents " (ibid., p.2). II distingue quatre grandes phases successives: I'arrivée (désorientation, découverte, image du désir, perte des schèmes de référence...), le repli (déconstruction identitaire, barrière linguistique, réapprentissage, doute...), la confrontation (désillusion, choc culturel, crise d'identité, conduite d'évitement...), et enfin, l'ouverture (autonomie, mobilisation, espoir...) C'est 
à cette dernière étape que nous pouvons mesurer l'accomplissement du processus d'adaptation.

On pourrait être tenté de rechercher un lien entre, d'une part, le vécu des jeunes et leurs expériences relationnelles primaires dans leurs pays d'origine et d'autre part, leur capacité à s'adapter à la société d'accueil. Cette approche, davantage psychologique, porterait sur le mode d'adaptation à la société d'accueil en fonction du style de liens d'attachement développé dans le contexte familial d'origine. Le schéma de Van Oudenhoven et Ofstra (2005) illustre ces mécanismes. (Dans Yaillian et Olie, 2014). Le fait d'avoir bénéficié au sein de la famille de liens d'attachements sécures, ou au contraire, d'avoir grandi dans une situation d'isolement ou de maltraitance, aurait un impact sur les facultés à s'adapter au nouveau contexte post-migratoire. S'arrêter sur le modèle familial semble pertinent au regard des différents contextes décrits par les jeunes rencontrés. Il s'agirait cependant d'étudier plus en profondeur la nature des liens entretenus aussi bien dans le pays d'origine que dans la société d'accueil. Nous n'avons néanmoins pas eu les moyens d'approfondir cette thématique qui aurait pu faire l'objet d'une étude à part entière.

Les services de l'Aide Sociale à l'Enfance, outre la mission de protection, tendent à faciliter l'adaptation à la société. Ils constituent généralement la première source d'étayage des Mineurs Non Accompagnés. Toutefois, bien souvent, et heureusement, ce ne sont pas les seuls repères pour les jeunes qui font aussi appel à d'autres personnes de confiance extérieures (professeurs, réseaux de militants, compatriotes, bénévoles, amis...) Semei évoque une expérience négative au sein d'un premier foyer où il ne se sentait pas bien traité. II a pu faire entendre sa parole grâce au réseau associatif et amical qu'il s'était constitué durant son recours judiciaire et c'est vers ces personnes qu'il se tourne en priorité aujourd'hui. Ou du moins, il sait qui solliciter en fonction de ses besoins.

"Six mois au squat, je me suis construit par les associations. Donc je connais un peu les trucs qu'il faut faire pour aboutir à quelque chose. J'ai fondé des réseaux. Même dans le service où je suis actuellement, ils me disent que quand je ne serai plus avec eux, si j'ai un problème, je pourrai les appeler. Tout cela aussi, ce sont des réseaux, à gauche, à droite. Pour le moment, je n'ai pas trop confiance en les structures parce que la chose que j'ai vécue avec le foyer ne me donne pas confiance. Et puis en plus, depuis que je suis arrivé, les dossiers, ce sont ma marraine ou l'avocat qui les gardent. "

Semei, ivoirien, 18 ans.

Malek sait aussi vers qui se tourner et n'accorde pas plus de légitimité aux professionnels qu'à son réseau amical. Pour lui, ce n'est pas la fonction qui importe, propos qui ressort dans de nombreux témoignages. 
"Je discute avec les éducateurs et s'ils n'arrivent pas à gérer, j'ai les amis, ou les pères des amis aussi. "

Malek, malien, 19 ans.

Pour Redei, enfin, la société d'accueil et ses fonctionnements s'enseignent dehors, dans la vie de tous les jours.

"Avec le temps, avec la vie dehors, en sortant, en marchant, en allant voir les amis, en lisant, en écoutant les journaux... A force de forger, on devient forgeron! Les gens ne te disent pas, mais quand tu fais un truc bien, cela se voit! C'est comme un enfant, quand il voit qu'on aime, il est content! Oui, on apprend tous les jours dans la vie! Celui qui dit un jour qu'il cesse d'apprendre, c'est qu'il est mort ! »

Redei, camerounais, 21 ans.

Ces étayages multiples facilitent l'adaptation à la société d'accueil, mais aussi, sans aucun doute une projection dans l'après dispositif.

\section{2. "Je veux être comme eux, entre eux." "}

Nous l'avons indiqué, s'intégrer à la société d'accueil est l'un des objectifs poursuivis par les Mineurs Non Accompagnés. Ils l'évoquent surtout en début de prise en charge, alors qu'ils ont encore peu de copains et que pour certains, la maîtrise du français fait défaut pour communiquer.

"Je ne veux pas être dans une classe et être toujours tout seul, rester dans ma culture et ne pas changer. J'aimerais être comme eux. Je ne peux pas changer ma peau mais j'aimerais être comme eux. La même culture. J'aimerais faire tout ce qu'ils font. Et j'aimerais être entre eux ".

Sekou, malien, 16 ans.

Sekou dit sa difficulté à s'intégrer. II explique que dans son collège situé en milieu rural, sur huit-cents élèves, ils ne sont que quatre " comme lui ", sous-entendu quatre Africains, les premiers d'ailleurs à être accueillis dans l'établissement. Si au début, les élèves venaient le voir et s'intéressaient à lui, il a créé peu de liens d'amitiés à ce jour et il se sent isolé. Mais l'effort est selon lui à fournir de son côté. C'est à lui d'aller vers les autres et non l'inverse.

"Tu te trouves tout seul, Noir au milieu de huit-cents élèves français! (rires) C'est dur, c'est dur! Mais on va y arriver quand même. C'est dur mais on va y arriver [...]. Chez nous, quand un jeune vient, on l'accueille. Ici, ils ne sont pas méchants, mais je ne sais pas comment cela se passe. Tout le monde est dans sa maison. Chez nous, tout le monde est dehors. On se connaît. Ici, c'est différent. Quand tu viens ici pour t'intégrer dans les classes, c'est difficile. [...] Maintenant, c'est à nous d'aller les voir car ce n'est pas eux qui se sont déplacés. C'est nous qui nous sommes déplacés pour venir ici. Quand on arrive parmi les gens, on doit être comme eux. On ne peut pas être comme eux naturellement mais on doit apprendre avec eux." Sekou, malien, 16 ans. 
Lors des entretiens, les échanges s'enclenchent facilement sur le thème des différences culturelles. Et ce qui revient en premier lieu, c'est la question du " respect ». Là-bas, on ne regarde pas les adultes dans les yeux, on ne répond pas à ses parents, ni aux professeurs. "Chez nous, c'est un manque de respect quand on regarde dans les yeux " nous dit Sekou. A l'inverse, le " s'il-te-plaît » ou le " merci », exigés ici, ne le sont pas forcément ailleurs. C'est bien connu, les Français adorent les formules de politesse. Dans d'autres pays, et il n'est pas nécessaire d'aller trop loin pour le constater, demander de façon polie peut suffire à induire le respect. Ces décalages, les Mineurs Non Accompagnés les observent et tentent de les prendre en compte dans leurs modes d'interaction. Si ce n'est pas le cas, les éducateurs se chargent de les y amener. Forcément, s'intégrer, c'est tenir compte des coutumes du pays $d^{\prime}$ accueil. Mais pour que la communication soit effective avec eux et pour les comprendre, les professionnels doivent eux aussi adopter une posture de décentrage. Ces jeunes ne mettent pas forcément les formes là où on les attendrait, peut-être, mais cela viendra petit à petit. II s'agit de ne pas s'en formaliser, de le signifier mais de s'en décaler pour laisser place à la relation.

Sekou se compare à ses camarades de classe. II n'est pas allé à l'école au Mali et dit avoir moins de connaissances qu'eux. II constate cependant que son expérience de vie, différente de la leur, lui a aussi beaucoup appris.

"On dit que quelqu'un qui a fait dix capitales connaît plus que celui qui a fait dix ans d'école. Eux, ils peuvent apprendre à l'école, mais ils ne voient pas. C'est cela que j'ai compris. "

Sekou, malien, 16 ans.

S'intégrer, n'est-ce pas se faire sa place au sein d'un groupe, en prenant de l'autre mais aussi en y laissant une trace ? Ne s'agit-il pas d'un échange ? Même si ce vécu n'est pas majoritaire, certains des jeunes interrogés ont l'impression de ne pas être bien accueillis. C'est le cas de Semei. Ce jeune, si jovial et ouvert à l'échange, entouré, apprécié des équipes... Ce jeune jugé autonome, qui est parvenu à s'adapter et à s'insérer, qui a trouvé un patron pour son apprentissage en boulangerie et a validé son BAFA $^{46} \ldots$ Ce jeune ne se sent pas bien accueilli en France. Et il clame non seulement son incompréhension, mais aussi la souffrance que ce vécu de rejet lui fait endurer. Rejet de la part d'un pays où il pensait pouvoir se faire une place. Sa place, de l'extérieur, on peut considérer qu'il se l'est faite et qu'on la lui a faite. Mais c'est audelà de l'insertion. L'accueil, pour lui, c'est dans le regard.

\footnotetext{
${ }^{46}$ Brevet d'Aptitudes aux Fonctions d'Animateur.
} 
"Je ne regrette pas d'être venu ici mais c'est l'accueil... Quand je vois l'accueil que vous nous portez, souvent, ça me fait mal. [...] A la police, on m'a insulté. Et le regard... Quand je sens cela, ça me fait mal. [...] D'avoir été mal accueilli, c'est comme si j'avais une certaine plaie quoi, qu'ils avaient mise dans moi. J'ai eu la rage. Parce que ça fait mal. Ça fait très, très, très, très, mal. Quand tu viens dans un pays, que tu parles français et qu'on te traite comme cela, qu'on t'insulte... C'est un choc ! Cela fait trop mal! [...] Chez nous, on disait que les Français étaient les grands-parents. Pourquoi quand un Africain parle portugais, le Portugais est fier ! Mais quand un Africain parle français... Mais pourquoi ? Ils nous voient toujours comme des esclaves quoi! [...] Je pensais que je serais bien accueilli ici, mais du coup ce n'est pas ça, c'est autre chose ".

Semei, ivoirien, 18 ans.

Rencontrer du monde est difficile ici pour Semei, en comparaison avec les modes relationnels dans son pays. II constate que les gens ont peur. II ne sait pas de quoi. Quand il pose la question, on lui répond que c'est la société. "Bah c'est que votre société est bizarre ! »conclut-il !

"Je ne sais pas ce qu'il y a en France, je ne sais pas ici, l'histoire d'ici, je ne sais pas ce qu'il y a eu ici, s'il y a eu des guerres, si les Noirs ont tué beaucoup de Blancs, je ne sais pas mais... Sincèrement, il y a un problème ici!"» Semei, ivoirien, 18 ans.

Il trouve paradoxal que les gens n'osent pas se parler dans la rue quand ils ne se connaissent pas, alors qu'ils le font sur les réseaux sociaux. II ironise aussi sur sa difficulté à rencontrer des filles et sur les refus qu'il se voit opposer.

"Toutes les Françaises sont en couple ici! Pourtant, à la télé, on dit qu'il y a plus de célibataires que de mariées!»

Semei, ivoirien, 18 ans.

L'intégration est donc difficile pour certains et source de souffrance. La plupart des jeunes, cependant, n’ont pas ce vécu, ou du moins, ils ne l'évoquent pas de la sorte. Ils peuvent dire entendre parfois des réflexions racistes, mais ne se formalisent pas. Comme Farhad, qui se remémore la question d'un camarade de classe : "Les étrangers viennent en France et l'État leur donne des appartements alors qu'il y a des Français à la rue. Pourquoi les Français acceptent cela ? " Ce garçon avait sûrement rencontré des problèmes avec un étranger, suppose Farhad.

Walid dit se sentir bien en France justement parce qu'il ne subit pas de discrimination, contrairement à l'Iran où il résidait avec sa famille.

"C'est pour cela que je suis content ici. II n'y a aucun problème. Je suis étranger, et alors, il n'y a rien du tout ! "

Walid, afghan, 18 ans. 
S'adapter à la société d'accueil et s'y intégrer, constituent deux enjeux majeurs pour toute personne en situation post-migratoire. Ces deux processus sont plus ou moins longs et difficiles, selon chacun, selon les contextes d'arrivée aussi. Qu'en est-il du sujet dans tout cela ? Au-delà des statuts et des rôles attribués, comment les Mineurs Non Accompagnés se construisent-ils en tant qu'individus autonomes ?

\subsection{Une individuation progressive}

L'individuation est entendue en sociologie comme le processus de production des individus dans un contexte de changements sociétaux et de modernité (Martucelli, 2005). La rencontre avec la société d'accueil produit pour notre public de nouvelles formes d'individualité. En effet, l'injonction à devenir un individu s'impose ici alors que dans la plupart des pays d'origine, c'est le collectif qui prime, avec une soumission à la loi du père.

A travers notre étude, nous avons pu constater une évolution dans le positionnement des jeunes à l'égard des adultes et des institutions à mesure qu'ils évoluaient dans leurs parcours au sein de l'Aide Sociale à l'Enfance. Nous avons souligné en effet que leurs priorités étaient en premier lieu de se poser et d'enclencher les projets scolaires. Une fois stabilisés dans les services d'accompagnement, ils commencent à interroger les fonctionnements institutionnels et réclament davantage de libertés, souhaitant vivre " comme les autres jeunes ».

Par ailleurs, en phase d'évaluation ou en début de prise en charge, ils remettent peu en cause les décisions. Ils n'ont pas de prise dessus et s'y conforment. De même que durant la traversée, ils sont agents d'un système qui leur échappe, comme en témoigne le vocabulaire utilisé : " c'est le juge qui décide ", "s'ils te prennent ", " on m'a envoyé ", " quand la décision tombe ", etc. Ils ne questionnent pas ce système, ils s'y soumettent. Souvent, si on les interroge de façon rétroactive sur la période transitoire entre l'évaluation et l'orientation, qui peut parfois durer de nombreux mois, ils expliquent qu'ils attendaient, sans expectatives particulières.

Peut-il en être autrement? Peut-on s'opposer, ou ne serait-ce qu'envisager d'autres alternatives à son arrivée dans une société que l'on ne connaît pas ? Semei décrit ce manque de "bagages " qui l'a empêché de faire valoir ses droits lors d'une première évaluation dans un département où il s'était arrêté. L'authenticité de ses documents administratifs ayant été contestée, il s'est vu opposer un refus de prise en charge, avec possibilité de prendre un avocat. "Mais pour quelqu'un qui vient 
d'arriver, en France, tu connais quoi ? Rien ! " II a donc quitté le département sans se saisir de la possibilité de déposer un recours.

De plus, suivre les conseils et les décisions des adultes, n'est-ce pas ce qui a été enseigné ? Les jeunes nous le disent, l'autorité du père n'est pas contestable. Même marié, on écoute les parents, et plus globalement, les aînés.

"Mon père m'a dit de toujours suivre l'ami. Comme il m'avait dit pour Michel: la manière dont tu me prends comme ton papa, Michel est ton papa. II faut obéir aux ordres de sa femme comme ta maman. II faut les respecter.".

Asad, guinéen, 15 ans.

Alors dans une société dont on ne connaît pas les fonctionnements, comment ne pas se conformer aux décisions ? Ce cadre qui leur est imposé et qui peut paraître contraignant n'est-il pas d'une certaine manière rassurant ? On s'en remet aux adultes et on se laisse porter, après une traversée éprouvante. Julien Bricaud le souligne, « certains jeunes refusent de décider quoi que ce soit les concernant et remettent leur avenir entre les mains des adultes à qui ils doivent respect et obéissance " (2012, p.79). Les réponses portant sur les choix d'orientation en sont une illustration.

"Je suis dans les bras du service".

Maamuni, sénégalais, 17 ans.

"Je crois que je n'aurai pas le choix. Moi, j'aime qu'une grande personne me donne des conseils pour savoir ce qui peut être bien pour moi, demain. C'est pour cela que je pense que je n'aurai pas le choix. Si la personne me dit que c'est cela qui est bon pour moi, si c'est une grande personne, je sais que c'est cela qui est bon. " Abdoulaye, ivoirien, 16 ans.

De la même manière, les règles sont peu discutées durant la phase d'évaluation ou le début de prise en charge. Hamidi souligne leur importance pour se construire.

"Les horaires, il y en a pour les repas, les passages des éducateurs, le retour à l'hôtel. En Algérie, il n'y avait pas d'horaires. Mais les règles, c'est bien! Les grands ont des règles, comme toi, tu as des règles, le travail, partir de la maison, les enfants... Pour une bonne vie, il faut les règles! Pareil pour les mineurs. Les règles, c'est important dans la vie! Pour une bonne vie! Pour tout le monde! En Algérie, il n'y a pas la loi ! Ce n'est pas bien!» Hamidi, algérien, 16 ans.

Osei est l'un des seuls à mettre en doute les discours et à adopter une posture de revendication. 
"A Bordeaux, je ne suis pas à l'aise. Je suis là au niveau du département, j'ai fait cinq mois ! J'ai fêté mes 16 ans dehors et jusqu'à présent, ils disent que je ne peux pas aller à l'école! Ils disent que si je pars à l'école, je ne pourrai pas avoir les papiers à 18 ans. Mais ça, ce n'est pas clair! Je le dis, ça ce n'est pas clair! J'écoute mais je ne crois pas moi! Ce n'est pas clair! Je vous dis ce que je pense ! [...] Moi, je ne suis pas un fou parce que j'ai étudié pour comprendre les choses! Moi, j'ai regardé la constitution française et un enfant de 15-16 ans, c'est obligatoire, il doit aller à l'école ! C'est obligé ça ! Comment peut-on me dire que non, je ne peux pas aller à l'école ? [...] IIs nous prennent pour des cons. Les gens, ils ne nous considèrent pas! Moi, je sais que je suis aimé ici, je sais que tout le monde m'aime ici, mais quand on regarde la réalité, c'est autre chose ! " Osei, guinéen, 16 ans.

A mesure que les jeunes évoluent au sein du dispositif de l'Aide Sociale à l'Enfance et s'installent dans la société d'accueil, ils s'affirment. Les premières revendications sont d'ordre financier et matériel et se font avec " détermination » constate Julien Bricaud, " quitte à mettre en cause la légitimité des professionnels " (2012, p.79). Première source de crispation avec les équipes, l'aspect financier est souvent central dans les interactions et interroge. Les explications peuvent être diverses : injonction à soutenir la famille et à rembourser les dettes de passage, désir de réaliser le mythe de la réussite sociale, manifestation de l'absence et nécessité de la combler, trace du passé colonialiste et demande de réparation (« vous nous devez bien cela ! »), mais aussi problème de compréhension de l'étendue des droits. Tenter d'en cerner les causes tout en se référant au cadre institutionnel permet aux professionnels de se décaler de ces questions qui viennent parasiter le suivi. Les jeunes, quant à eux, demandent des explications.

"Il n'y a pas de négociation. Comment peut-on avoir une discussion sans négociation ?"

Ange, guinéen, 17 ans.

A mesure que la relation éducative se met en place et que la confiance s'installe, ces questions se font plus silencieuses et l'accompagnement prend tout son sens. De quoi s'agit-il alors ? Comme avec tous les jeunes, Mineurs Non Accompagnés ou non, on tente de les amener vers "l'autonomie », et pas uniquement matérielle.

"Mon éducatrice m'a dit : "Dans ta vie, tu vas faire comme tu veux. Tout ce que tu vas faire, comme le métier, personne ne te forcera et on te demandera ce que tu veux ». En Guinée, ce sont tes parents qui te disent ce que tu vas faire. Pouvoir choisir, c'est bien! Tu vas faire comme tu veux! C'est bien! Regarde ma coupe! Moi, en Guinée, je ne me coiffe pas comme cela!" Hamad, guinéen, 16 ans. 
Les engagements pris auprès du Conseil Départemental placent généralement au premier plan les démarches d'insertion socio-professionnelle. Dans l'article intitulé "Écrits de jeunes en quête de statut», plus de deux-cents lettres de jeunes demandant des contrats jeunes majeurs depuis les années 1970 sont étudiées afin de faire ressortir l'évolution des exigences à leur égard. "Alors que l'envie de poursuivre une scolarité, le besoin d'hébergement, l'aide financière ou le besoin d'être en relation suffisaient à l'obtention du statut de jeune majeur, aujourd'hui, les jeunes doivent justifier la demande par une défaillance familiale et/ou personnelle. Ils doivent donner un sens à leur prise en charge. " (Guimard et Petit-Gats, 2010). II est exigé qu'ils fassent preuve d'une "volonté manifeste de s'insérer" et témoignent de "leur relation aux travailleurs sociaux ". On leur donne la possibilité d'être " acteurs de leur histoire ». Mais "faire appel à leur seule responsabilité, les juger selon leur attitude, c'est oublier les raisons qui les ont amenés à être pris en charge dans des établissements éducatifs et négliger leurs parcours. [...] N'est-ce pas trop exiger que de faire comme s'ils possédaient les ressources nécessaires pour faire librement des choix ? » (Ibid.) Cette question est celle que se posent ou doivent se poser les équipes intervenant auprès des Mineurs Non Accompagnés, au même titre qu'avec tous les publics en difficulté. Prendre en compte la capacité d'agir de la personne ne signifie pas oublier ses fragilités à un instant t. Le rôle des éducateurs est de favoriser une compréhension de l'environnement pour que l'individu puisse s'y mouvoir librement. II doit avoir la possibilité de se constituer sujet libre de faire des choix tout en pouvant se reposer sur un cadre rassurant et étayant. C'est tout l'enjeu de l'accompagnement éducatif.

\section{CONCLUSION DE LA TROISIÈME PARTIE}

Les Mineurs Non Accompagnés au prisme de la sociologie de la jeunesse... Ce sont des adolescents comme les autres dans cette phase de transition entre deux âges où l'on n'est ni enfant, ni adulte. Il est question de passage entre deux statuts, ceux de mineur et de majeur, qui ne prennent sens que dans la société d'accueil. Être mineur ne renvoie pas spécifiquement à un âge mais à un besoin d'étayage et au fait de ne pas se sentir "responsable ». Devenir adulte signifie avoir la capacité de s'assumer et éventuellement de participer à l'économie familiale. Accéder à ce statut passe par la mise en place de stratégies facilitatrices. Ces dernières peuvent s'avérer bénéfiques mais ne doivent pas entacher l'inscription dans les liens de filiation, nécessaire à la construction du sujet au sein de la nouvelle société. 


\section{CONCLUSION}

Notre étude portait sur la rencontre des Mineurs Non Accompagnés avec la société d'accueil et ses institutions. Nous avons cherché dans un premier temps à comprendre ce qui les poussait au départ, en nous intéressant aux contextes de vie, mais aussi aux aspirations et aux attentes familiales. II s'agissait alors d'analyser comment ces jeunes s'arrangeaient des rôles attribués là-bas et ici. Le détour par les classifications a mis en évidence la proportion élevée de "mineurs-aspirants » et l'émergence d'une nouvelle catégorie que nous avons nommée les «mineurséloignés ». Mais derrière les différents projets migratoires, c'est avant tout une quête de statut qui se dessine, celui d'aîné social, ou encore d'individu moderne. Prendre la route, c'est déjà grandir. Réussir socialement dans un ailleurs, avec la possibilité de contribuer à l'économie familiale ou à son prestige, c'est grandir d'autant plus. Sur ce dernier aspect, il est ressorti que le mandat familial n'était pas toujours présent et que lorsqu'il existait effectivement, il pouvait être implicite. De plus, cette responsabilité à l'égard des familles n'est pas incompatible avec la possibilité de se réaliser personnellement. Intérêts collectifs et individuels s'articulent. C'est au fil de I'installation et de l'adaptation que s'opère le processus d'individuation. Il est à mettre en lien avec les positions d'agent et d'acteur, traduisant la façon dont ces jeunes prennent part à leurs trajectoires, de même que les marges de manœuvre dont ils disposent ou non au fil de leurs parcours. Nous n'avions pas forcément identifié ces mécanismes initialement et c'est tout l'intérêt de l'étude.

Par ailleurs, en interrogeant le passage à l'âge adulte, nous avons abordé sous l'angle culturel les questions de frontière d'âge, mais aussi de responsabilisation et de projection dans l'avenir. C'était une manière de traiter la thématique des rôles sociaux en faisant un pas de côté, plus léger, mais riche de sens. Est adulte, donc, celui qui prend en charge. II n'est nullement fait référence ici à l'âge administratif. La frontière mineur/majeur est entendue en termes de dépendance/autonomie. Solliciter une mesure éducative revient à énoncer un besoin de soutien. Et pour être entendu, il faut être reconnu mineur. C'est la première exigence à laquelle se confrontent ces jeunes. En effet, au même titre que les lois françaises sur l'immigration reposent sur une politique de filtrage, accéder au dispositif de l'Aide Sociale à l'Enfance est soumis à critères. Et bien que la mission de protection puisse être vécue comme restrictive des libertés, en opposition aux habitudes de vie passées, la logique stratégique consistant à diminuer son âge n'a pas forcément d'impact sur l'individu vu que cette notion ne prend pas sens. En revanche, celle qui consiste à nier les attaches familiales pour répondre au second critère d'isolement comporte plus de risques dans la construction du sujet. Pour revenir à notre hypothèse de départ, si l'identité intégratrice du sujet 
risque bel et bien d'être menacée, c'est davantage par ces questions de filiation que par les stratégies liées à l'âge.

Il semble nécessaire que soit clarifiée cette dimension au niveau législatif pour que les liens familiaux soient maintenus et puissent être travaillés dans les services éducatifs. Nous voyons naître des projets associatifs innovants allant dans cette direction et ils sont à valoriser. Par ailleurs, favoriser l'accueil et l'accompagnement des publics migrants adultes en France, avec des possibilités supérieures d'accès à un titre de séjour et à une autorisation de travail, éviterait des glissements vers le secteur de la protection de l'enfance.

La question des attentes émerge rapidement dans la prise en charge des Mineurs Non Accompagnés. Tout comme l'idée d'un décalage avec la réalité rencontrée sur place. Nous en avons fait une hypothèse. Notre étude a démontré que contrairement à nos représentations, ces jeunes n'avaient pas forcément connaissance en amont du dispositif de l'Aide Sociale à l'Enfance. Nous sommes tout de même tentée de garder certaines réserves sur cet aspect, au même titre que nous l'avons fait sur les contextes de départ. Annoncer sa venue en France dans l'intention d'être pris en charge pourrait en effet traduire une visée stratégique qui serait dénoncée. La catégorie socioculturelle influe sur le niveau d'information et il n'est pas impossible que les plus privilégiés, mais aussi ceux qui étaient au contact de la mobilité, aient eu écho de certains éléments de réalité. Au-delà de cette question, il est certain que les représentations sont nombreuses sur l'Europe et que cet ailleurs fantasmé ne trouve pas toujours écho une fois sur place où se présentent maints obstacles, d'où effectivement une certaine désillusion et souvent une forme d'incompréhension.

L'exigence forte à laquelle se confrontent ces jeunes après celle de l'évaluation est la régularisation administrative, à laquelle se juxtapose rapidement celle d'autonomisation. Là encore, il est question de statut. Une fois admis dans les services, une période de répit s'offre à eux avec un début de stabilisation et la possibilité d'enclencher les démarches d'insertion. En tant que mineurs, ils peuvent s'appuyer sur les équipes, voire se laisser porter. Mais à l'approche de la majorité, les démarches s'accélèrent et l'obtention du titre de séjour conditionne la suite du parcours. II importe alors d'accéder au statut de " majeur en situation régulière » sur le territoire, puis de "majeur en situation régulière et autonome ". Notre hypothèse stipulait que malgré le décalage entre les représentations et les exigences de la société d'accueil, ces dernières facilitaient la réalisation des projets. Les lois sur l'immigration tendent à se durcir ces dernières années et les Mineurs Non Accompagnés ne sont pas épargnés. Nous avons souligné l'inquiétude générée à l'approche de la majorité et l'impact négatif qu'ont les obstacles administratifs sur les parcours individuels. En ce sens, les 
exigences législatives en matière de droit au séjour peuvent constituer un frein à l'installation. Ce n'est nullement une découverte mais il est essentiel de l'avoir à l'esprit pour prendre la mesure du vécu de ces jeunes.

Si l'on revient maintenant au dispositif de protection de l'enfance, malgré son caractère parfois contraignant, il soutient les intérêts individuels en facilitant l'adaptation et l'insertion dans la société d'accueil. De la même manière, l'injonction à l'autonomie une fois majeur, même perçue comme précipitée et déstabilisante, va dans ce sens. Aspirations et exigences institutionnelles trouvent donc une issue commune dans l'autonomisation. Néanmoins, nous n'avons pu rencontrer que deux jeunes sortis du dispositif pour évaluer, avec du recul, leur adaptation et la réalisation de leurs projets. D'après les retours qui sont faits dans les services éducatifs, le "dehors", comme disent certains, peut s'avérer compliqué. Outre le fait de se retrouver seul, il n'est pas rare que soient rencontrées des difficultés d'accès au logement, de gestion des démarches administratives ou du budget. A cette étape du parcours, le "mandat " familial, qui n'était pas forcément perçu comme un poids, peut devenir plus oppressant. L'autonomisation visée par le Conseil Départemental porte sur la capacité de l'individu à s'assumer, mais le contexte économique actuel ne lui permet pas forcément d'accéder au confort de vie auquel il aspirait, ni de soutenir en parallèle sa famille. Et s'il le fait, ce peut être à son détriment. Ce n'est qu'une fois seuls que les jeunes mesurent cette réalité. Le statut de migrant ayant réussi sa vie n'est pas si simple à conquérir. Le dispositif de protection de l'enfance, donc, par son caractère exigeant, facilite effectivement la réalisation des projets individuels, mais peut-être en partie seulement.

S'intéresser aux trajectoires post-institutionnelles des Mineurs Non Accompagnés pourrait faire l'objet d'une étude à part entière dans la continuité de celle-ci. En plus d'explorer le processus d'adaptation, nous pourrions réinterroger la réaction face aux attentes familiales à cette nouvelle étape du parcours où soutenir n'est plus qu'une affaire de projection. Une réflexion autour des besoins de ce public paraît également essentielle. II s'agirait de penser de nouveaux modes d'accompagnements au-delà des 21 ans. Des accueils de jour permettant à la fois de maintenir un lien social et de proposer un soutien aux démarches de façon inconditionnelle, comme il en existe pour les publics marginalisés, pourraient être développés.

Notre étude était relativement large et la reprendre en quelques conclusions semble réducteur. Nous avons eu cette même impression au moment de passer de la retranscription des entretiens à l'analyse du matériau, qui laissait de côté de précieux contenus. Si ce travail aura permis de donner la parole aux Mineurs Non Accompagnés, 
les verbatim sélectionnés pour leur caractère significatif ne font malheureusement pas honneur à toutes les personnes interrogées, certaines n'ayant pas pu être citées. C'est le jeu de l'enquête et la rigueur imposée par ce type de travail nous y conduit. Les propos se lisent néanmoins entre les lignes, à travers les tendances mises en évidence. Et si le procédé peut paraître frustrant, les rencontres et les histoires de vie laissent une trace en filigrane. Alors que nous partions sur un échantillon de vingt personnes, nous en avons finalement rencontré trente-trois. Le guide d'entretien portait sur un nombre assez important de thématiques qu'il aurait peut-être fallu réduire pour se recentrer sur certaines dimensions finalement privilégiées dans l'écrit. Mais c'est au fil des échanges que s'est précisée l'approche. D'autre part, au moment de traiter les données, nous avons constaté que toutes les questions n'avaient pas été approfondies avec la même intensité selon les entretiens, ce que l'on peut regretter. C'est cependant le qualitatif qui veut cela. Quant au paramètre de l'authenticité, il a été très compliqué à manier dans la rédaction de la première partie portant sur les contextes de départ. Cette dimension, centrale dans la prise en charge des Mineurs Non Accompagnés, venait mettre en péril la fiabilité de l'étude. II serait intéressant de savoir de quelle façon s'en sont arrangés les chercheurs à l'origine des typologies sur ce public. Pour notre part, nous décaler de ces données a été délicat mais c'est une preuve d'honnêteté qui nous est apparue nécessaire.

Mener la recherche dans le domaine d'intervention qui était le nôtre aura à la fois enrichi et complexifié la démarche. Partir d'observations de terrain, mais aussi faire un pas de côté pour laisser place à l'inattendu dans la rencontre, décrypter et faire parler les matériaux, analyser des données qui ne corroboraient pas forcément le savoir construit professionnellement, étaient toute la difficulté de ce travail. Lorsque I'on intervient auprès du public des Mineurs Non Accompagnés, on est plongé dans un univers à part, tel le monde migratoire, avec ses propres codes. On acquiert des réflexes, un langage, des pratiques spécifiques que l'on fait siennes mais qui pour quelconque novice pourraient sembler surprenantes. Ne serait-ce que l'expression " évaluation de la minorité » interpelle. Dans quel autre contexte est-on amené à interroger l'âge déclaré ? A l'entrée d'une boîte de nuit peut-être, mais une présentation de la carte d'identité suffit à confirmer et il ne viendrait à l'idée d'aucun agent de sécurité de vérifier la qualité du tampon apposé. Un monde spécifique, donc, duquel il est important de se décaler parfois pour ne pas se laisser happer, au risque d'en oublier le sens de son intervention. C'est un public qui agace autant qu'il fascine de par sa complexité.

Enfin, nous intéresser à l'expérience des Mineurs Non Accompagnés au fil de leurs prises en charge au sein de l'Aide Sociale à l'Enfance nous a amenée à pousser la porte des institutions, mais aussi des associations plus informelles. Outre l'opportunité 
qui nous a été ainsi offerte de découvrir leurs fonctionnements, tout comme les enjeux, c'est la notion de parcours qui a été mise en évidence. Il suffit d'interroger les équipes des différents établissements pour s'apercevoir que les représentations sur le public divergent. Il s'agit pourtant du même. Ce qui diffère, c'est le type de structure, certes, mais surtout, l'étape à laquelle se trouvent les jeunes dans leurs parcours d'adaptation, les besoins et les attentes évoluant. Cette dimension ne nous paraissait pas aussi évidente avant l'étude. Elle nous a sauté aux yeux lors du stage au CAMINA où nous nous sommes trouvés face à des jeunes en grande souffrance et peu à même de prendre des décisions pour eux-mêmes, contrairement à ceux du SRSA, déjà installés dans la société d'accueil, qui pour la plupart voyaient leurs situations se stabiliser et affirmaient leurs désirs. C'est toute la richesse de cette recherche. Ce processus a été mis en évidence non pas en suivant les mêmes personnes sur plusieurs années, ce dont nous n'avions pas les moyens, mais en rencontrant des jeunes d'une même catégorie sociologique à différentes étapes, que tous seraient amenés à franchir. Les associations diversifiant leurs modes de prise en charge en s'appuyant sur cette dimension de parcours ont vu juste.

Cette étude s'est proposée de porter un autre regard sur le public des Mineurs Non Accompagnés à travers le prisme des rôles sociaux. Elle ne prétend nullement contenir des informations ou des vérités auxquelles les professionnels de terrain n'auraient pas accès. De fait, ceux qui sont au plus proche de ces jeunes connaissent leurs situations. Il s'agissait cependant de recourir à nouvelle grille de lecture de ces trajectoires de vie, d'un point de vue sociologique et par certains aspects, anthropologique. S'intéresser aux aspirations et aux vécus des attentes à leur égard a ouvert sur leurs subjectivités. La lecture de cet écrit peut permettre aux professionnels d'adopter une vision plus globale des parcours et d'élargir leurs représentations sur ce qui anime ces acteurs. Nous avons lancé quelques pistes de réflexion autour de nouvelles formes d'accompagnement au-delà des 21 ans, du soutien aux relations familiales, mais aussi d'une diversification des types d'accueil pour favoriser des parcours individualisés évolutifs. Différents aspects de l'étude ont suscité chez nous un vif intérêt et pourraient donner lieu à de nouvelles recherches plus ciblées. Nous avons eu un aperçu des trajectoires post-institutionnelles à travers deux témoignages et approfondir cette question s'inscrirait dans la continuité de notre travail, tout en donnant de précieuses indications sur d'éventuelles orientations politiques à mettre en œuvre. Qui sait ? II n'est pas impossible que nous recroisions les protagonistes de cette étude dans quelques années! 


\section{SOURCES ET RÉFÉRENCES BIBLIOGRAPHIQUES}

\section{OUVRAGES :}

- Bricaud, J. (2012), Accueillir les jeunes migrants, Lyon, Edition Chronique Sociale, Collection Comprendre la société.

- Dubet, F. (1991), Les lycéens, Paris, Editions du Seuil, Collection L'épreuve des faits.

- Dubet, F. (1994), Sociologie de l'expérience, Paris, Editions du Seuil, Collection La couleur des idées.

- Galland, O. (1991), Sociologie de la jeunesse, Paris, Edition Armand Colin.

- Legault, G. (2000), L'intervention interculturelle, Québec, Edition Gaëtan Morin, collection Pratiques Professionnelles

- Weber, M. (1922), Economie et société, Tome 1, Paris, Edition Pocket, Collection Pocket Agora.

- Wylie, L. (1968), Un village du Vaucluse, Paris, Edition Gallimard, Collection Témoins.

\section{CHAPITRES D'OUVRAGES :}

- Ma Mung, E. (2009) « Le point de vue de l'autonomie dans l'étude des migrations internationales : penser de l'intérieur les phénomènes de mobilité », dans Dureau F. et Hily, M.A., "Les mondes de la mobilité, exploration d'un paradigme », Rennes, Presses de l'Université de Rennes, pp. 25-38, sur https://hal.archives-ouvertes.fr/hal-01075325

\section{ARTICLES DE REVUES :}

- Benoit De Coignac, A. et Baubet, T. (2013), «Transes et construction identitaire chez les mineurs isolés étrangers ", Adolescences, T31, $n^{\circ} 3$, pp.613-623 sur https://www.researchgate.net/publication/270119972_Transes_et_construction_iden titaire_chez_les_mineurs_isoles_etrangers

- Blanchard, E. et Rodier, C. (2016), "Crise migratoire : ce que cachent les mots ", Plein droit, n¹11, pp.3-6, sur https://www.cairn.inforevue-plein-droit-2016-4-page-3.htm/

- Bourdieu, P. (1978), " La jeunesse n'est qu'un mot », entretien avec Métailié, A.-M., paru dans Les jeunes et le premier emploi, Paris, Association des Ages, pp.520-530, sur http://www.homme-moderne.org/societe/socio/bourdieu/questions/jeuness.html - Chicchelli, V. (2001), "Les jeunes adultes comme objet théorique ", Recherches et prévisions, n65, pp.5-18, sur https://www.persee.fr/doc/caf_1149-1590_2001_num_ 65_1_961

- Coenen-Huther, J. (2005), "Heurs et malheurs du concept de rôle social ", Revue européenne des sciences sociales, T.43, $\mathrm{n}^{\circ} 132$, pp.65-82 sur https://journals. openedition.org/ress/328 
- Dièye Gueye, D. (2007), "Les mobilisations pour le départ migratoire ", Migration et société, $\mathrm{n}^{\circ} 109$, pp.11-26, sur https://www.cairn.info/revue-migrations-societe-2007-1page-11.htm

- Dubar, C. (2007), "Les sociologues face au langage et à l'individu ", Langage et société, $n^{\circ} 121-122$, pp. 29-43, sur https://www.cairn.info/revue-langage-et-societe2007-3-page-29.htm

- Duvivier, E. (2010), «Entre installation et poursuite de la mobilité. Analyse des trajectoires post-institutionnelles de jeunes isolés étrangers accueillis à l'Aide Sociale à l'Enfance ", Migrations Société, 2010/3-4, n²9-130, pp.243-256, sur https://www.cairn.info/revue-migrations-societe-2010-3-page-243.htm

- Etiemble, A. et Zanna, O. (2013), «Des typologies pour faire connaissance avec les mineurs isolés étrangers et mieux les accompagner. Synthèse ", https://www.infomie. net/IMG/pdf/synthese_-_actualisation_typologie_mie_2013-2.pdf

- Fouquet, T. (2007), "L'imaginaire migratoire ", Autrepart, n41, Editeur Presses de Sciences Po, pp.83-98 sur https://www.cairn.info/revue-autrepart-2007-1-page83.htm

- Guimard, N. et Petit-Gats, J. (2010), "Ecrits de jeunes en quête de statut ", Recherches familiales, $n^{\circ} 7$, pp.115-125, sur https://www.cairn.info/revue-recherchesfamiliales-2010-1-page-115.htm

- Martuccelli, D. (2005), "Les trois voies de l'individu sociologique ", Espacestemps.net, sur htpps://www.espacestemps.net/articles/trois-voies-individusociologique/

- Mauger, G. (2010), "Jeunesse : essai de construction d'objet ", Presses de Sciences Po, $n^{\circ} 56$, pp. 9-24 sur https://www.cairn.info/revue-agora-debats-jeunesses-2010-3page-9.htm

- Mauger, G. (2001), « La jeunesse n'est qu'un mot. A propos d'un entretien avec Pierre Bourdieu ", Agora débats / jeunesses, $\mathrm{n}^{\circ} 26$, pp.137-142, sur https://www.persee.fr/doc/agora_1268-5666_2001_num_26_1_1924

- Pagnon, M.J. (2011), « Les trajectoires des jeunes migrants ou leur installation en France ", Hommes et migrations, pp.88-96, sur http://journals.openedition.org/ hommes migrations/745

- Roche, A. (2006), "Les jeunesses au prisme de la sociologie. État des lieux ", Siècles, n²4, pp.9-23, sur http://journals.openedition.org/

- Sayad, A. (1977), "Les trois "âges" de l'émigration algérienne en France », Actes de la recherche en sciences sociales, Vol. 15, pp. 59-79, sur https://www.persee.fr/ doc/arss_0335-5322_1977_num_15_1_2561

- Sayad, A. (septembre 1999), "Immigration et "pensée d'Etat" ", Actes de recherche en sciences sociales, vol.129, Délits d'immigration, pp.5-14, sur https://www.persee.fr/ doc/arss_0335-5322_1999_num_129_1_3299 
- Senovilla-Hernández, D. (2014), "Analyse d’une catégorie juridique récente : le mineur étranger non accompagné, séparé ou isolé ", Revue européenne des migrations internationales, vol. 30, n¹, pp.17-34, sur http://journals.openedition.org/remi/6732 - Senovilla-Hernández, D. (2010), « Mineurs Non Accompagnés et séparés en Europe. Une analyse comparative de l'application de la Convention Internationale des Droits de I'Enfant dans six pays", Migrations Société, n¹29-130, pp.99-114, sur https://www.cairn.info/revue-migrations-societe-2010-3-page-99.htm

- Séraphin, G. (2001), «Etre jeune à Douala. Le difficile chemin de la conquête statutaire ", Agora débats / jeunesses, n²6, pp.123-135, sur https://www.persee.fr/ doc/ agora_1268-5666_2001_num_26_1_1923

- Témime, E. (1999), «Comprendre l'immigration. Quelques notes en mémoire d'Abdelmalek Sayad : un sociologue hors du commun ", Revue du monde musulman et de la Méditerranée, n85-86, Le post-islamisme, pp.265-273, sur https://www.persee. $\mathrm{fr} / \mathrm{doc} / \mathrm{remmm}$ 0997-1327_1999_num_85_1_2649

- Vacchiano, F. (2014), «A la recherche d'une citoyenneté globale. L'expérience des adolescents migrants en Europe ", Revue européenne des migrations internationales, vol.30, $n^{\circ} 1$, pp.59-81, sur http://journals.openedition.org/remi/6761

\section{RAPPORTS ET THÈSES :}

- Clavé-Mercier, A. ( $\left.2^{\circ} 14\right)$, Des états et des "Roms » : une anthropologie du sujet entre transnationalisme et politiques d'intégration de migrants Bulgares en France, Thèse de doctorat en ethnologie, Bordeaux, Ecole doctorale Sociétés, politique, santé publique, sur https://tel.archives-ouvertes.fr/tel-01418979/document.

- CNAPE (février 2018), Accompagner et accueillir les MNA au regard de leurs besoins, sur http://www.justice.gouv.fr/art_pix/1_CNAPE_Rapport_Accompagnement_accueil_ MNA_2018.pdf

- Doineau, E. et Godefroy, J-P. (28 juin 2017), Rapport d'information sur la prise ne charge des Mineurs Non Accompagnés, fait au nom de la commission des affaires sociales et transmis au Sénat, sur https://www.senat.fr/notice-rapport/2016/r16-598notice.html

- Etiemble, A. et Zanna, O. (juin 2013), Des typologies pour faire connaissance avec les MIE et mieux les accompagner. Synthèse, collectif Topik, sur https://www.infomie.net/ IMG/pdf/synthese_-_actualisation_typologie_mie_2013-2.pdf

- France Terre D’Asile (2017), L'accueil et la prise en charge des Mineurs Isolés Etrangers en France, sur http://www.infomie.net/IMG/pdf/la_prise_en_charge_et_ I_accueil_des_mie_pages.pdf 
- Ministère de la justice (2016), Foire Aux Questions. Mineurs Non Accompagnés, sur http://www.justice.gouv.fr/art_pix/FAQ_mineurs_isoles.PDF

- ONPE (février 2017), Mineurs non accompagnés : quels besoins et quelles réponses ?, dossier thématique coordonné par Jamet, L. et Keravel, E., sur https://www.onpe. gouv.fr/system/files/publication/dossier_mna_web2.pdf

- UNHCR (décembre 2018), C'est bien qu'on nous écoute, sur https://www.unhcr.org/ $\mathrm{fr}-\mathrm{fr} / 5 \mathrm{c} 17 \mathrm{~cd} 034$.

- Van de Velde, C. (2007), "Devenir adulte : quatre modèles européens ", Agora Débats / Jeunesses, $n^{\circ} 45$, pp.22-31, sur http://www.ressourcesjeunesse.fr/IMG/pdf/ agora45VanDeVelde.pdf

\section{CONFÉRENCES :}

- Kobanda, D., Tanon, F., Vitturi, L. (2011), « Table ronde 1 : Les causes des migrations des mineurs isolés étrangers ", Migrations et société, n¹36, pp.18-24, publié dans https://www.cairn.Info/revue-migrations-societe-2011-4-page-18.htm

- Oui, A. (septembre 2012), "Les jeunes vulnérables face au système d'aide sociale. Politiques publiques, pratiques professionnelles et trajectoires de jeunes", intervention au colloque de l'EHESP, publié par l'ONED, sur https://www.onpe.gouv.fr/ system/files/publication/20130220_ehesp_ao.pdf

- Yaillian, A. et Olie, E. (2014), " Immigration et théorie de l'attachement ", $12^{\mathrm{e}}$ congrès de I'Encéphale, sur file:///C:/Users/am\%C3\%A9lie/Downloads/PO-432-YAILIAN.pdf

\section{FILMS DOCUMENTAIRES :}

- Diallo, T.S (2018), Difficile à raconter, Guinée, Le Grenier des Ombres.

- Fandio, P. (2017), Migrants - retour d'enfer, Hemisphere Media Production Africa, France.

- Oujdi, R. (2016), J'ai marché jusqu'à vous - récits d'une jeunesse exilée, France, Comic Strip Production.

\section{OUTILS MÉTHODOLOGIQUES :}

- Quivy, R. et Van Campenhoudt, L. (1995), Manuel de recherches en sciences sociales, Paris, Edition Dunod.

- Kaufmann, J.-C. (1996), L'entretien compréhensif, Paris, édition Nathan, collection 128.

- Jounin, N. (2014), Voyage de classes, Paris, Edition La Découverte.

- Olivier de Sardan, J.-P. (1995), « La politique du terrain. Sur la production de données en anthropologie.", Revue Enquête, 1/1995, pp.71-109, sur https://journals. openedition.org/enquete/263 


\section{TABLE DES MATIÈRES}

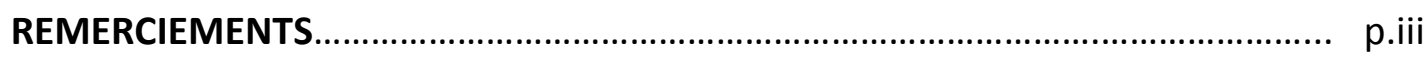

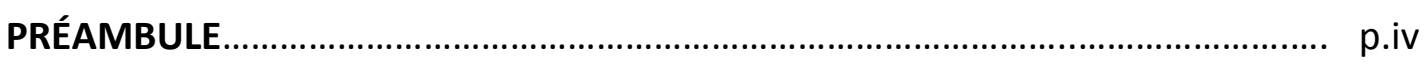

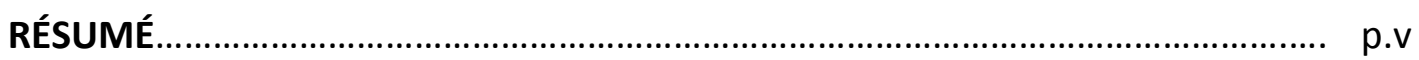

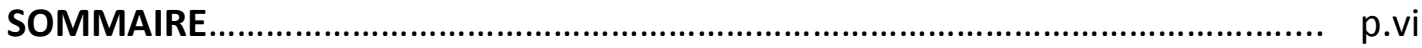

INTRODUCTION........................................................................................... p.1

$\checkmark$ Les Mineurs Non Accompagnés, actualité et cadre législatif..................... p.1

$\checkmark$ Un public spécifique faisant émerger de nouvelles interrogations........... p.4

$\checkmark$ Des institutions aux réseaux informels.................................................... p.8

$\checkmark$ Des récits, des tendances......................................................................... p. p.12

$\checkmark$ Quelques histoires de vie introductives................................................ p.14

PARTIE 1. UNE MIGRATION AUX COUTOURS PARTICULIERS.............. p.17

1. UNE EXPÉRIENCE NOUVELLE DE LA MIGRATION............................................ p.17

1.1. Culture de la migration et imaginaire migratoire ........................................... p.17

1.2. Des contextes de départ diversifiés.................................................................. p.20

1.3. La migration comme traduction d'un rapport moderne au monde................ p. 23

2. TANTÔT ACTEUR, TANTÔT AGENT DU PROJET MIGRATOIRE............................ p. p.28

2.1. Projet migratoire et autonomie......................................................................... p.28

2.2. Projet individuel versus projet familial.......................................................... p.29

2.3. Rôles vis-à-vis de la famille......................................................................... p... p. p.

3. PRENDRE LA ROUTE OU LA SUIVRE............................................................ p.34

3.1. Immersion dans un « monde migratoire "..................................................... p.34

3.2. Une traversée facteur de fragilisation bien que formatrice............................ p.36

3.3. Des niveaux d'information disparates........................................................ p. p.38

CONCLUSION DE LA PREMIÈRE PARTIE.......................................................... p.40

PARTIE 2. RENCONTRE AVEC UNE SOCIETE D'ACCUEIL EXIGEANTE.... p.41

1. DES DROITS ET DES DÉMARCHES.............................................................. p.41

1.1. " Les papiers, il n'y a pas ça chez nous ! " ........................................................ p.41

1.2. Un accès spécifique au droit commun............................................................. p.43

1.3. Une évaluation de la minorité légitimée mais contraignante...........................p.45 
2.1. Être étayé dans un contexte inconnu........................................................... p.47

2.2. Enclencher les projets d'insertion et $s^{\prime}$ intégrer........................................... p. p.50

2.3. Une fois installé, reconquérir ses libertés........................................................ p.52

3. EXIGENCES DE RÉGULARISATION ET D'AUTONOMISATION........................... p.55

3.1. De la méconnaissance du droit au séjour à la conscientisation des enjeux.....p.55

3.2. Une insertion socio-professionnelle par étapes.............................................. p.5

3.3. Une injonction à l'autonomie pressante............................................................. p.60

CONCLUSION DE LA DEUXIÈME PARTIE............................................................... p.64

PARTIE 3. UNE JEUNESSE DANS L'ENTRE-DEUX .................................... p.65

1. ENTRE DEUX ÂGES, ENTRE DEUX CULTURES................................................... p.65

1.1. L'âge des incohérences statutaires................................................................ p.65

1.2. Âge administratif versus âge des responsabilités.............................................. p.68

1.3. Devenir adulte là-bas et ici................................................................................ p. 70

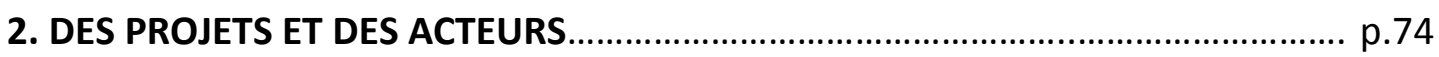

2.1. Différentes logiques d'action...................................................................... p. p. 74

2.2. L'âge qu'il faut.............................................................................................. p.77

2.3. Nature des liens avec la famille...................................................................... p. p 80

3. ADAPTATION, INTÉGRATION ET INDIVIDUATION........................................... p.81

3.1. Des étayages multiples pour s'adapter........................................................... p.81

3.2. « Je veux être comme eux, entre eux " ............................................................. p.84

3.3. Une individuation progressive......................................................................... p.87

CONCLUSION DE LA TROISIÈME PARTIE........................................................... p.90

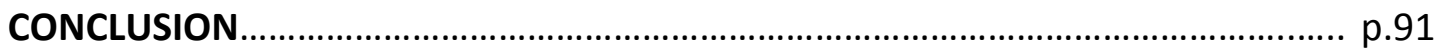

SOURCES ET RÉFÉRENCES BIBLIOGRAPHIQUES.................................................... p.96

TABLE DES MATIÈRES................................................................................... p. 100

ANNEXES

Annexe 1 : Cadre législatif de la prise en charge des Mineurs Non Accompagnés..p.I

Annexe 2 : Tableau synoptique de la recherche........................................................ p.III

Annexe 3 : Guide d'entretien................................................................................ p.IV

Annexe 4 : Tableau récapitulatif des entretiens....................................................... p.VII

Annexe 5 : Une illustration d'entretien............................................................... p.XI 


\section{ANNEXE 1 : CADRE LÉGISLATIF DE LA PRISE EN CHARGE DES MNA}

\section{La Convention Internationale des Droits de l'Enfant du 20 novembre 1989}

Article 20: "Tout enfant qui est temporairement ou définitivement privé de son milieu familial, ou qui dans son propre intérêt ne peut être laissé dans ce milieu, a droit à une protection et à une aide spéciales de l'Etat. "

Article 2 : "Les Etats parties s'engagent à respecter les droits qui sont énoncés dans la présente convention et à les garantir à tout enfant relevant de leur juridiction, sans distinction aucune, indépendamment de toute considération de race, de couleur, de sexe, de langue, de religion, d'opinion politique ou autre de l'enfant ou de ses parents ou représentants légaux, de leur origine nationale, ethnique ou sociale, de leur situation de fortune, de leur incapacité, de leur naissance ou de toute autre situation ».

$\checkmark \quad$ La loi du 5 mars 2007 réformant la protection de l'enfance a modifié les dispositions suivantes du Code de l'Action Sociale et des Familles :

Article L112-3 : " La protection de l'enfance vise à garantir la prise en compte des besoins fondamentaux de l'enfant, à soutenir son développement physique, affectif, intellectuel et social et à préserver sa santé, sa sécurité, sa moralité et son éducation, dans le respect de ses droits. La protection de l'enfance a également pour but de prévenir les difficultés que peuvent rencontrer les mineurs privés temporairement ou définitivement de la protection de leur famille et d'assurer leur prise en charge. »

Cet article stipule que les Mineurs Non Accompagnés relèvent de la protection de l'enfance.

Article L223-2: "En cas d'urgence et lorsque le représentant légal du mineur est dans l'impossibilité de donner son accord, l'enfant est recueilli provisoirement par le service qui en avise immédiatement le procureur de la république. »

Les services de l'Aide Sociale à l'Enfance sont chargés de recueillir provisoirement les Mineurs Non Accompagnés.

$\checkmark \quad$ La loi $n^{\circ}$ 2016-297 du 14 mars 2016 relative à la protection de l’enfant a inséré deux nouveaux alinéas au code civil :

\section{Article 375-5 du code civil :}

"Lorsqu'un service de l'aide sociale à l'enfance signale la situation d'un mineur privé temporairement ou définitivement de la protection de sa famille, selon le cas, le procureur de la République ou le juge des enfants demande au ministère de la justice de lui communiquer, pour chaque département, les informations permettant l'orientation du mineur concerné. »

"Le procureur de la République ou le juge des enfants prend sa décision en stricte considération de l'intérêt de l'enfant, qu'il apprécie notamment à partir des éléments ainsi transmis pour garantir des modalités d'accueil adaptées. » 
$\checkmark$ La loi $n^{\circ}$ 2016-297 du 14 mars 2016 relative à la protection de l'enfant a inséré également un nouvel article au code de l'action sociale et des familles :

Article L221-2-2 : " Pour permettre l'application du $3^{\mathrm{e}}$ alinéa de l'article 375-5 du code civil, le président du Conseil Départemental transmet au ministère de la justice les informations dont il dispose sur le nombre de mineurs privés temporairement ou définitivement de la protection de leur famille dans le département. Le ministère de la justice fixe les objectifs de répartition proportionnée des accueils de ces mineurs entre les départements, en fonction des critères démographiques et d'éloignement géographique.»

Cette loi donne un fondement légal au dispositif de répartition territoriale des Mineurs Non Accompagnés.

Pour mettre en œuvre ces dispositions, le décret en conseil d’Etat $n^{\circ} \mathbf{2 0 1 6 - 8 4 0}$ du 24 juin 2016 fixe les conditions d'accueil, d'évaluation et d'orientation des mineurs privés temporairement ou définitivement de la protection de leur famille (clé de répartition). Le président du Conseil Départemental statue sur la reconnaissance ou non de la qualité de mineur privé temporairement ou définitivement de la protection de sa famille à partir du rapport de synthèse et de l'avis du service chargé de l'évaluation. En cas de doute sur l'authenticité des documents, le préfet peut être interpelé. En cas de doute sur l'âge, l'autorité judiciaire est saisie.

L'arrêté ministériel du 17 novembre 2016 pris en application du décret n²016-840 du 24 juin 2016 fixe les modalités d'évaluation de la minorité et de l'isolement familial des personnes se déclarant mineures privées temporairement ou définitivement de la protection de leur famille.

Au sein de la Sous-Direction des Missions de Protection Judiciaire et d'Education (SDMPJE), la Mission Mineurs Non Accompagnés (MMNA) coordonne le dispositif national de mise à l'abri, d'évaluation et d'orientation des Mineurs Non Accompagnés. Elle fait fonctionner la cellule nationale d'appui à l'orientation.

\section{SOURCES}

- Foire Aux Questions. Mineurs Non Accompagnés (Ministère de la justice).

http://www.justice.gouv.fr/art_pix/FAQ_mineurs_isoles.PDF

- Arrêté du 17 novembre 2016.

https://www.legifrance.gouv.fr/affichTexte.do?cidTexte=JORFTEXT000033420352\&categorie Lien=id

- Directives du parlement européen et du conseil du 13 décembre 2011.

https://www.gisti.org/spip.php?article2675

- MNA : focus sur le décret du 24 juin 2016.

http://www.justice.gouv.fr/justice-des-mineurs-10042/mineurs-non-accompagnes-focus-

sur-le-decret-du-24-juin-2016-29227.html 


\begin{tabular}{|c|c|c|c|c|}
\hline THÉMATIQUE & PROBLÉMATIQUE & HYPOTHÈSES & PLAN & MÉTHODOLOGIE \\
\hline 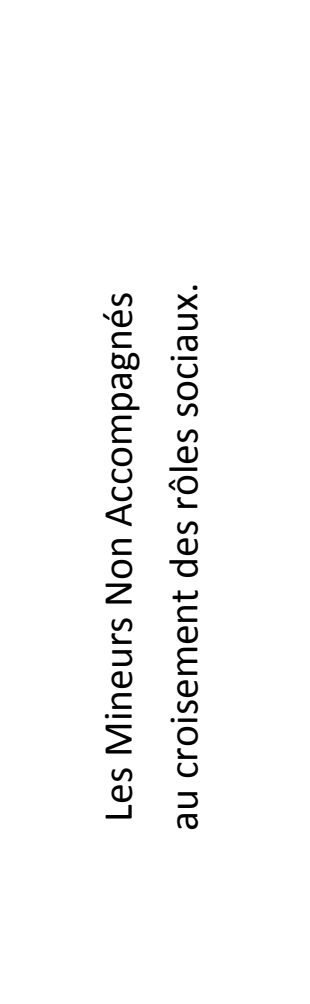 & 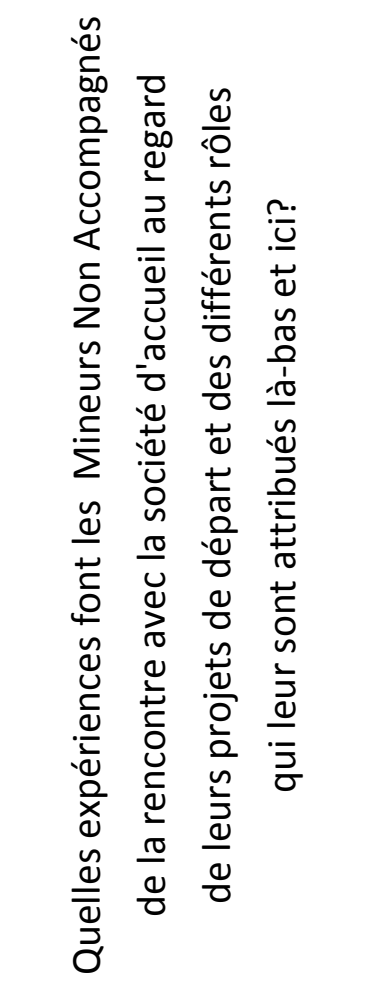 & $\begin{array}{l}\text { 1. La société d'accueil présente } \\
\text { des exigences en décalage avec } \\
\text { les représentations et les } \\
\text { attentes des Mineurs Non } \\
\text { Accompagnés, tout en } \\
\text { favorisant la réalisation de } \\
\text { leurs projets. } \\
\text { 2. Les Mineurs Non } \\
\text { Accompagnés adoptent des } \\
\text { stratégies d'action pouvant } \\
\text { menacer leur « identité } \\
\text { intégratrice ». }\end{array}$ & $\begin{array}{l} \\
\text { Partie } 3 \text { : Une jeunesse dans } \\
\text { l'entre-deux. }\end{array}$ & 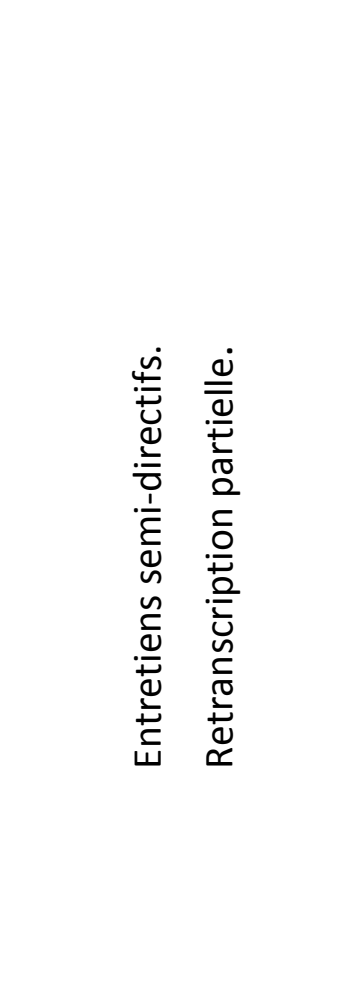 \\
\hline
\end{tabular}




\section{ANNEXE 3 : GUIDE D'ENTRETIEN}

Remarque : Ce guide a servi de support mais il a été adapté à chaque type de structure.

\section{QUESTION GÉNÉRALE}

Objectif : savoir comment la personne vit globalement sa situation post-migratoire et si le regard qu'elle porte sur sa trajectoire est positif ou négatif.

Que pouvez-vous nous dire de votre situation actuelle?

\section{HISTOIRE FAMILIALE ET PROJET MIGRATOIRE}

Objectif : mettre en parallèle l'histoire familiale avec le projet de départ et savoir si les stratégies d'acteurs sont en lien avec des attentes familiales.

Pourriez-vous vous présenter?

1. Quel âge avez-vous ? D'où venez-vous ? Comment viviez-vous dans votre pays ? Que faisiez-vous?

2. Pourquoi avez-vous quitté votre pays ? Votre départ a-t-il été pensé et organisé avec votre famille?

3. Pour quelles raisons avez-vous choisi la France ? Qui vous en avait parlé et à quel moment ? Quelles informations aviez-vous reçues ?

4. Êtes-vous en contact avec votre famille ? Aimeriez-vous pouvoir l'aider plus tard ? Compte-t-elle sur vous ? Vous sentez-vous libre de parler de vos relations familiales? Si ce n'est pas le cas, pourquoi et comment le vivez-vous?

\section{RAPPORT AU DISPOSITIF D'AIDE SOCIALE À L'ENFANCE}

Objectif : savoir quelles étaient les attentes vis-à-vis de l'Aide Sociale à l'Enfance et comment est vécu le dispositif.

Comment vivez-vous le système de protection de l'enfance ?

1. Y a-t-il des foyers dans votre pays ? Si oui, comment fonctionnent-ils ? Y a-t-il des éducateurs et si c'est le cas, quel est leur rôle ? Avant de venir en France, saviez-vous que les services de protection de l'enfance existaient ? Si oui, qui vous en avait parlé et à quel moment ? Qu'est-ce que vous imaginiez ? Pourquoi avez-vous fait une demande d'accompagnement? 
2. Saviez-vous qu'il y avait une phase d'évaluation de la minorité ? Comment l'avezvous ressentie? Et votre premier accueil ? Comment vivez-vous les exigences administratives et institutionnelles? Comment vous sentez-vous dans votre service actuel ? Avez-vous de bonnes relations avec l'équipe et les autres jeunes ? Y at-il des éléments qui vous surprennent et si oui, lesquels ? Comment percevez-vous les règles de fonctionnement ? Avez-vous l'impression que vos besoins sont satisfaits ? Si ce n'est pas le cas, pouvez-vous préciser?

3. Qu'est-ce qu'un éducateur selon vous ? Pensez-vous avoir besoin des éducateurs? Pourquoi ? Est-ce qu'ils vous aident ? Si oui, à quoi ? Si non, pour quelles raisons ?

\section{ETRE ENFANT, ETRE ADULTE}

Objectif : savoir ce que représente dans la culture de la personne le passage à la majorité, à mettre en lien avec les injonctions de la société d'accueil.

C'est quoi pour vous être enfant et être adulte?

1. Jusqu'à quel moment est-on considéré comme un enfant dans votre pays ? Que font les enfants et quels rôles ont-ils ? Est-ce qu'ils bénéficient là-bas d'une protection particulière ? Pourquoi protège-t-on les enfants en France ? Qu'en pensez-vous ?

2. Dans votre pays, parle-t-on de la période de l'adolescence ? Comment sont considérés les jeunes et ont-ils des rôles particuliers au sein de la famille ? Sont-ils différents là-bas et ici ? Si oui, en quoi ? Trouvez-vous qu'ils sont plus matures là-bas ou ici ?

3. À quel âge a lieu la majorité dans votre pays ? Qu'est-ce que cela change là-bas de devenir majeur ? Sur quels critères est-on considéré comme un adulte ? Comment se passe le passage à l'âge adulte ? Peut-on ou doit-on faire de nouvelles choses ?

4. Savez-vous ce que cela changera pour vous de devenir majeur ici? Quelles informations avez-vous sur le droit au séjour ? Êtes-vous inquiet?

5. L'âge d'une personne a-t-il de l'importance pour vous dans les relations ? Certains jeunes sont amenés à déclarer un âge différent du leur, comment pensez-vous qu'ils vivent cette situation ? Est-il compliqué pour eux de ne pas pouvoir donner leur âge réel et si oui, en quoi ? Quels sont selon vous les avantages et les inconvénients à se déclarer mineurs?

\section{INSCRIPTION DANS SON ENVIRONNEMENT}

Objectif : repérer comment la personne comprend et évolue dans son environnement.

Comment vous sentez-vous à Bordeaux ? 
1. Y a-t-il des choses qui vous surprennent en France?

2. Comment s'organisent actuellement vos journées ? Que faites-vous ?

3. Qui est présent autour de vous ? Avez-vous des amis? Sur qui pouvez-vous compter? Qui allez-vous voir en cas de besoin ? Pourquoi avez-vous confiance en ces personnes en particulier? Avez-vous l'impression d'être bien accueilli en France ? Vous sentez-vous intégré ?

4. Trouvez-vous que les jeunes rencontrés ici pensent de la même façon que vous ? Avez-vous les mêmes envies ou préoccupations ? Lesquels par exemple ? Ont-ils des habitudes de vie ou des fonctionnements différents ? $Y$ a-t-il des manières de se comporter en France ou des codes qui vous surprennent ? Pouvez-vous donner des exemples? Si vous comparez ce que vous faisiez enfant dans votre pays avec la vie des enfants ici, voyez-vous des différences? Et pour les jeunes? Les adultes ? Qu'en pensez-vous?

5. Est-ce que vous savez comment fonctionnement les institutions de service public (Caisse Primaire d'Assurance Maladie, Caisse d'Allocations Familiales, Conseil Départemental, écoles...) ? Avez-vous déjà rempli des dossiers administratifs ? Est-ce que vous comprenez à quoi ils servent?

\section{SITUATION ACTUELLE ET PROJECTIONS}

Objectif : savoir comment la personne vit sa situation actuelle et si elle porte un regard positif ou négatif sur son avenir.

\section{Comment percevez-vous votre situation ?}

1. Comment vous sentez-vous dans votre vie aujourd'hui ? Qu'est-ce qui se passe bien et qu'est-ce qui est difficile ? Avez-vous l'impression d'être confronté à des exigences et si oui, lesquelles?

2. Comment imaginez-vous la fin de la prise en charge à l'Aide Sociale à l'Enfance ? Savez-vous à quel moment elle s'arrêtera ? Cela vous inquiète-t-il ? Que signifie pour vous être autonome ? Pensez-vous que la prise en charge à l'Aide Sociale à l'Enfance vous aidera à être autonome et si oui, en quoi ? Auprès de qui pourrez-vous avoir du soutien quand vous aurez quitté le dispositif?

3. Quelle vie aimeriez-vous avoir plus tard ? Où aimeriez-vous habiter ? Envisagez-vous de repartir dans votre pays ? Qu'est-ce que vous aimeriez faire ? Pensez-vous pouvoir mener à bien vos projets ? Ce serait quoi pour vous, réussir votre vie? 


\begin{tabular}{|c|c|c|c|c|c|c|c|c|}
\hline \multicolumn{4}{|c|}{ PERSONNE INTERROGÉE } & \multirow{2}{*}{$\begin{array}{l}\text { DISPOSITIF } \\
\text { D'ACCUEIL }\end{array}$} & \multicolumn{4}{|c|}{ ENTRETIEN } \\
\hline Prénom & $\begin{array}{l}\text { Sexe } \\
M / F\end{array}$ & Nationalité & Âge & & Date et durée & Lieu & $\begin{array}{l}\text { Prise de notes } \\
\text { ou enregistré }\end{array}$ & Tonalité \\
\hline $\begin{array}{c}\text { Oussy } \\
\text { Malek } \\
\text { Issan } \\
\text { François }\end{array}$ & $\begin{array}{l}M \\
M \\
M \\
M\end{array}$ & $\begin{array}{c}\text { Malienne } \\
\text { Malienne } \\
\text { Guinéenne } \\
\text { Camerounaise }\end{array}$ & $\begin{array}{l}18 \text { ans } \\
19 \text { ans } \\
18 \text { ans } \\
18 \text { ans }\end{array}$ & $\begin{array}{l}\text { Prado SRSA } \\
\text { Prado SRSA } \\
\text { Prado SRSA } \\
\text { Prado SRSA }\end{array}$ & $\begin{array}{l}28 / 01 / 2019 \\
1 \mathrm{~h} 17\end{array}$ & $\begin{array}{l}\text { Une salle du } \\
\text { SRSA }\end{array}$ & Enregistré & $\begin{array}{l}\text { Le film suscite des } \\
\text { réactions. Dynamisme et } \\
\text { nombreux échanges. }\end{array}$ \\
\hline 2. Malek & $M$ & Malienne & 19 ans & Prado SRSA & $\begin{array}{c}01 / 02 / 2019 \\
42 \mathrm{mn}\end{array}$ & $\begin{array}{c}\text { Colocation } \\
\text { institutionnelle }\end{array}$ & Enregistré & $\begin{array}{l}\text { Peu enclin à développer, } \\
\text { assez fermé. }\end{array}$ \\
\hline 3. Issan & $M$ & Guinéenne & 18 ans & Prado SRSA & $\begin{array}{c}01 / 02 / 2019 \\
42 \mathrm{mn}\end{array}$ & $\begin{array}{l}\text { Appartement } \\
\text { institutionnel }\end{array}$ & Enregistré & $\begin{array}{l}\text { Très pénible et douloureux. } \\
\text { Se cache les yeux. }\end{array}$ \\
\hline 4. François & M & Camerounaise & 18 ans & Prado SRSA & $\begin{array}{l}30 / 01 / 2019 \\
1 \mathrm{~h} 16\end{array}$ & $\begin{array}{l}\text { Appartement } \\
\text { institutionnel }\end{array}$ & Enregistré & $\begin{array}{l}\text { La traversée est centrale } \\
\text { dans le discours. }\end{array}$ \\
\hline 6. Léa & $\mathrm{F}$ & Congolaise & 20 ans & Prado SRSA & $\begin{array}{c}29 / 04 / 2019 \\
?\end{array}$ & $\begin{array}{l}\text { Appartement } \\
\text { institutionnel }\end{array}$ & Prise de notes & $\begin{array}{l}\text { Dans l'échange. Réservée } \\
\text { sur le contexte de départ. }\end{array}$ \\
\hline 7. Farhad & $\mathrm{M}$ & Afghane & 17 ans & Prado SRSA & $\begin{array}{c}20 / 02 / 2019 \\
1 \text { h07 }\end{array}$ & $\begin{array}{c}\text { Chambre en } \\
\text { famille logeuse }\end{array}$ & Enregistré & Dans l'échange. \\
\hline 8. Bakkir & $M$ & Malienne & 17 ans & Prado SRSA & $\begin{array}{c}01 / 03 / 2019 \\
?\end{array}$ & $\begin{array}{l}\text { Bureau du } \\
\text { SRSA }\end{array}$ & Prise de notes & $\begin{array}{c}\text { Ne parle pas du passé. Peu } \\
\text { de réflexivité. }\end{array}$ \\
\hline
\end{tabular}




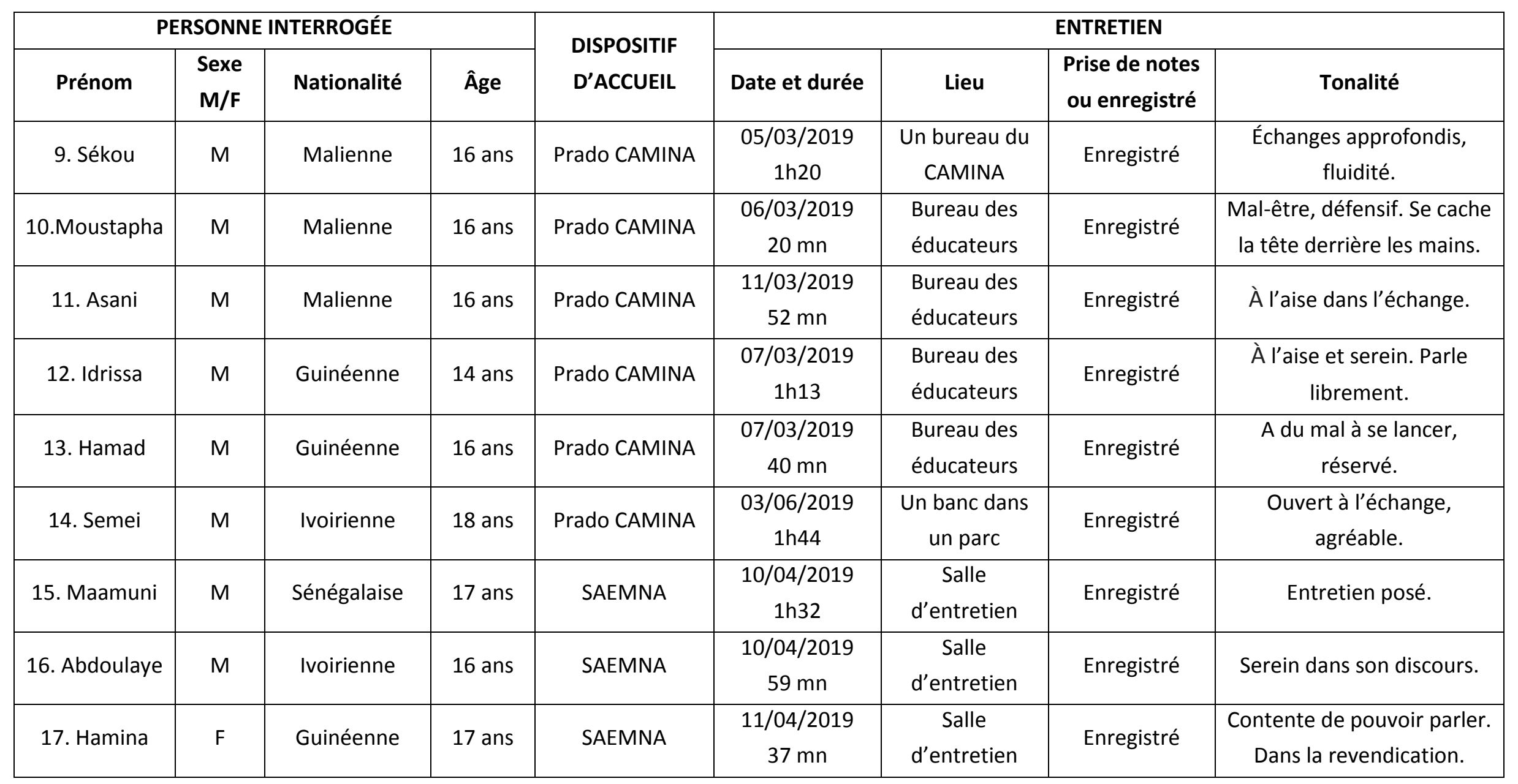




\begin{tabular}{|c|c|c|c|c|c|c|c|c|}
\hline \multicolumn{4}{|c|}{ PERSONNE INTERROGÉE } & \multirow{2}{*}{$\begin{array}{l}\text { DISPOSITIF } \\
\text { D'ACCUEIL }\end{array}$} & \multicolumn{4}{|c|}{ ENTRETIEN } \\
\hline Prénom & $\begin{array}{l}\text { Sexe } \\
M / F\end{array}$ & Nationalité & Âge & & Date et durée & Lieu & $\begin{array}{c}\text { Prise de notes } \\
\text { ou enregistré }\end{array}$ & Tonalité \\
\hline 18. Ouma & M & Guinéenne & 17 ans & SAEMNA & $\begin{array}{c}10 / 04 / 2019 \\
1 \mathrm{~h} 32\end{array}$ & $\begin{array}{c}\text { Salle } \\
\text { d'entretien }\end{array}$ & Enregistré & Fragilité apparente. \\
\hline 19. Osei & M & Guinéenne & 16 ans & SAEMNA & $\begin{array}{c}11 / 04 / 2019 \\
1 \mathrm{~h} 23\end{array}$ & $\begin{array}{c}\text { Salle } \\
\text { d'entretien }\end{array}$ & Enregistré & Assuré. Revendicatif. \\
\hline 20. Hamidi & M & Algérienne & 16 ans & SAEMNA & $\begin{array}{c}11 / 04 / 2019 \\
37 \mathrm{mn}\end{array}$ & $\begin{array}{c}\text { Salle } \\
\text { d'entretien }\end{array}$ & Enregistré & $\begin{array}{l}\text { Peu à l'aise avec le français } \\
\text { mais dans l'échange. }\end{array}$ \\
\hline 21. Harambee & M & Guinéenne & 17 ans & SAEMNA & $\begin{array}{c}12 / 04 / 2019 \\
1 \mathrm{H} 09\end{array}$ & $\begin{array}{c}\text { Salle } \\
\text { d'entretien }\end{array}$ & Enregistré & $\begin{array}{l}\text { Défensif au début puis } \\
\text { exprime son vécu. }\end{array}$ \\
\hline 22. Asad & M & Guinéenne & 15 ans & SAEMNA & $\begin{array}{c}12 / 04 / 2019 \\
54 \mathrm{mn}\end{array}$ & $\begin{array}{c}\text { Salle } \\
\text { d'entretien }\end{array}$ & Enregistré & $\begin{array}{c}\text { Exprime ses ressentis et ses } \\
\text { difficultés. }\end{array}$ \\
\hline 23. Vinza & M & Ivoirienne & 16 ans & Squid & $\begin{array}{c}30 / 04 / 2010 \\
?\end{array}$ & Salle du squat & Prise de notes & $\begin{array}{l}\text { Contexte saugrenu. Mal- } \\
\text { être et impuissance. }\end{array}$ \\
\hline 24. Saïd & M & Ivoirienne & 16 ans & Squid & $\begin{array}{c}30 / 04 / 2010 \\
?\end{array}$ & Salle du squat & Prise de notes & Posé et réfléchi. \\
\hline 25. Bayou & M & Guinéenne & 16 ans & Squid & $\begin{array}{c}30 / 04 / 2010 \\
?\end{array}$ & Salle du squat & Prise de notes & $\begin{array}{l}\text { Difficulté à élaborer. Met } \\
\text { mal à l'aise dans la relation. }\end{array}$ \\
\hline 26. Issam & M & Gambienne & 16 ans & Squid & $\begin{array}{c}01 / 05 / 2019 \\
?\end{array}$ & Salle du squat & Prise de notes & Très fragile et réservé. \\
\hline 27. Lumumba & M & Guinéenne & 16 ans & Squid & $\begin{array}{c}01 / 05 / 2019 \\
?\end{array}$ & Salle du squat & Prise de notes & Mal-être et impuissance. \\
\hline
\end{tabular}




\begin{tabular}{|c|c|c|c|c|c|c|c|c|}
\hline \multicolumn{4}{|c|}{ PERSONNE INTERROGÉE } & \multirow{2}{*}{$\begin{array}{l}\text { DISPOSITIF } \\
\text { D'ACCUEIL }\end{array}$} & \multicolumn{4}{|c|}{ ENTRETIEN } \\
\hline Prénom & $\begin{array}{l}\text { Sexe } \\
M / F\end{array}$ & Nationalité & Âge & & Date et durée & Lieu & $\begin{array}{c}\text { Prise de notes } \\
\text { ou enregistré }\end{array}$ & Tonalité \\
\hline 28. Ange & M & Guinéenne & 17 ans & R d'accueil & $\begin{array}{l}04 / 06 / 2019 \\
1 \mathrm{~h} 28\end{array}$ & $\begin{array}{l}\text { Salle de classe } \\
\text { du foyer }\end{array}$ & Enregistré & $\begin{array}{c}\text { Elaboration et réflexivité. } \\
\text { Très agréable. }\end{array}$ \\
\hline 29. Lamin & M & Ivoirienne & 16 ans & R d'accueil & $\begin{array}{c}\text { 04/06/2019 } \\
?\end{array}$ & $\begin{array}{c}\text { Salle de classe } \\
\text { du foyer }\end{array}$ & Prise de notes & Revendicatif. \\
\hline 30. Chilemba & M & Guinéenne & 16 ans & R d'accueil & $\begin{array}{c}\text { 07/06/2019 } \\
?\end{array}$ & $\begin{array}{l}\text { Salle de classe } \\
\text { du foyer }\end{array}$ & Prise de notes & $\begin{array}{l}\text { Sur la défensive au début, } \\
\text { puis dans l'échange. }\end{array}$ \\
\hline 31. Jahali & M & Guinéenne & 16 ans & R d'accueil & $\begin{array}{c}\text { 07/06/2019 } \\
?\end{array}$ & $\begin{array}{l}\text { Salle de classe } \\
\text { du foyer }\end{array}$ & Prise de notes & $\begin{array}{l}\text { Malaise, méfiance et } \\
\text { incompréhension. }\end{array}$ \\
\hline 32. Anton & M & Kosovare & 26 ans & $\begin{array}{l}\text { Sorti du } \\
\text { dispositif }\end{array}$ & $\begin{array}{l}02 / 04 / 2019 \\
40 \mathrm{mn}\end{array}$ & $\begin{array}{c}\text { Bungalow du } \\
\text { chantier }\end{array}$ & Enregistré & $\begin{array}{c}\text { Contexte particulier, le } \\
\text { sien. A l'aise et agréable. }\end{array}$ \\
\hline 33. Redei & M & Camerounaise & 21 ans & $\begin{array}{l}\text { Sorti du } \\
\text { dispositif }\end{array}$ & $\begin{array}{c}09 / 05 / 2019 \\
1 \mathrm{~h} 13 \mathrm{mn} \\
07 / 06 / 2019 \\
32 \mathrm{mn}\end{array}$ & $\begin{array}{l}\text { Appartement } \\
\text { personnel }\end{array}$ & Enregistré & $\begin{array}{c}\text { Entre complicité et } \\
\text { représentation. }\end{array}$ \\
\hline
\end{tabular}


Entretien avec Sékou, le 05 mars 2019, dans un bureau du CAMINA ${ }^{47}$.

\section{PRÉSENTATION}

2. CONTEXTE DE DÉPART ET RELATIONS FAMILIALES

\section{VÉCU DE SA SITUATION ACTUELLE}

\section{4. ÊTRE ADULTE}

\section{PRÉSENTATION}

« Je m'appelle Sekou G. J'ai 16 ans. Je viens d'avoir 16 ans dimanche passé. Je suis au collège, en quatrième. Je viens du Mali, de la région de Kayes. Cela fait un an et trois ou quatre mois que je suis là. J'étais à Paris avant de venir à Bordeaux. Je suis arrivé ici au mois de mars de l'année dernière ". C'est à Bordeaux qu'il a été évalué.

« II y a un moment, j'étais en difficultés, mais au jour d'aujourd'hui c'est bon, cela se passe bien. Ça ne se passe pas bien, bien, bien, mais ça se passe un peu bien. II $y$ avait des jours où je n'arrivais pas à dormir, où je passais toute la nuit à pleurer. C'était à cause du juge. "

\section{CONTEXTE DE DÉPART ET RELATIONS FAMILIALES}

\section{Vie au Mali et départ}

Quand Sekou avait 3 ou 4 ans, son père n'avait pas assez de moyens pour le garder à la maison alors il l'a " donné » à un " maître " afin qu'il puisse apprendre le Coran. Son village natal était dans la région de Kayes. "Ils $m^{\prime}$ ont envoyé pour aller à Macina " (au Nord du Mali). "J'ai passé toute ma vie là-bas. À un moment, je me suis dit que ça n'allait pas, que ça ne marchait pas bien ». On lui enseignait le Coran mais il ne savait pas ce que cela signifiait car il ne parlait pas l'arabe. «Tu ne peux pas rester chez quelqu'un qui ne veut pas, qui ne peut rien faire pour toi. Le Coran, c'est bien, mais tu ne peux pas vivre avec ça ».

Au sujet du " maître ", " on le paye mais pas avec l'argent direct ». Sekou devait sortir pour mendier et rapporter de quoi manger. Ils étaient nombreux dans la maison, une centaine. Il faisait tout là-bas. Un jour où il était parti en Mauritanie avec le fils du « maître » pour mendier, Sekou a décidé avec un " pote » de ne pas rentrer et il s'est enfui. Il s'est ensuite rapproché du Maroc, avant de prendre le bateau pour l'Espagne. Au départ, il pensait rester en Mauritanie mais des gens lui ont parlé du Maroc et de la

\footnotetext{
${ }^{47}$ Carré d'Accueil pour les Mineurs Non Accompagnés.
} 
possibilité là-bas de trouver du travail. " On ne sait pas comment ça se passe ". "Nous aussi on est rentré dedans comme ça ". En Mauritanie, il y a la traite d'esclaves. Les arabes "ne sont pas gentils du tout ». Un transporteur de ciment les a emmenés. Au début, il a refusé car ils étaient des "gamins". Mais ils ont finalement réussi à partir avec lui.

\section{Représentations de la France et de l'Europe}

Sekou ne connaissait pas la France. Mais dans son village, il y a beaucoup de gens qui partaient là-bas et quand ils revenaient en vacances au Mali, tout le monde voyait qu'ils étaient bien habillés alors ils voulaient partir aussi. "Quand on voit les choses, nous-mêmes on sait que l'Europe et l'Afrique, c'est différent. On sait qu'ici, ils vivent mieux que chez nous ". À tous les niveaux. «Par exemple, moi je suis né là-bas, je n'ai jamais été à l'école, je n'ai jamais fait deux ans ou même un mois complet avec mes parents. II y a plusieurs jeunes comme moi. Là, je vois les jeunes ici, mes potes au collège, qui vivent avec leurs parents. Le matin, ils les déposent en voiture, le soir, ils viennent les chercher... "

Sekou ne savait pas qu'il y avait des foyers avant de venir. "Arrivé au Maroc, j'ai pensé que quand je serais en Europe, ma vie allait changer. C'est ça que j'avais dans ma tête. Mais je ne savais pas comment cela allait se passer... Je n'ai pas pensé qu'il y avait des foyers... " Si des Maliens lui demandaient des conseils, il leur dirait de ne pas venir. "Là, c'est la galère ". II a des "potes " à Bordeaux qui dorment dans la rue. "C'est impossible ! » II évoque le départ, le trajet, la violence et une fois ici, " ça recommence à zéro. " "Etre dans la rue, ce n'est pas facile. " II n'imaginait pas qu'il serait amené à dormir dehors. En Afrique, on ne le croirait pas s'il racontait !

"Au foyer, on est bien mais il n'y a pas grand-chose ". Sekou aimerait avoir son appartement. Il pensait qu'il pourrait trouver un travail. Avant, il ne savait pas qu'il fallait des titres de séjours mais il a été accompagné à $\mathrm{ALIFS}^{48}$ et on lui a expliqué. La demande de titre de séjour l'inquiète, mais " pas trop en fait ". II ne veut pas " se prendre la tête pour rien, alors que ça ne va pas encore arriver ».

\section{Relations avec sa famille}

Sekou a une sœur plus jeune, qu'il n'a rencontré qu'une fois. Son père a aussi des enfants avec ses deux autres femmes mais Sekou ne sait rien d'eux. Chaque femme vit dans une maison différente. Ses parents ne savaient pas ce qu'il vivait chez le « maître " mais ne pouvaient de toute façon rien faire pour lui. Durant sept années, il n'a pas été en contact avec eux, n'avait ni téléphone, ni la possibilité d'envoyer du courrier. II ne sait pas s'ils avaient des nouvelles de lui ou pas. Peut-être par le biais du

\footnotetext{
${ }^{48}$ Association du Lien Interculturel Familial et Social.
} 
« maître » lorsqu'il se rendait dans le village pour mendier. Ils n’ont pas été informés de son départ pour l'Europe.

Maintenant, Sekou est un peu en lien avec ses parents. " Ils sont contents mais pas trop ». Sa mère lui pose des questions, lui demande avec qui il est. Elle s'inquiète, ne sait pas comment cela se passe pour lui. II n'a pas eu souvent l'occasion de communiquer avec eux car le portable vient juste d'arriver au foyer. Et puis dans son village, il n'y a pas de téléphone. Pour s'appeler, il faut qu'ils se donnent rendez-vous et que sa mère aille en ville. II aimerait leur envoyer un téléphone pour pouvoir communiquer. II voudrait aller les voir mais pas pour rester là-bas. Et faire venir sa mère ? "Elle ne va pas accepter". Et puis, " elle ne peut pas, elle est vieille maintenant $"$.

\section{VÉCU DE SA SITUATION ACTUELLE \\ Scolarité}

"Au collège, j'aimerais apprendre beaucoup mais comme je n'ai jamais été à l'école... " Sekou explique être allé à l'école coranique, " pour lire le coran seulement, dans la tête ". II apprenait pas cœur mais ne savait ni lire ni écrire. En arrivant, il ne parlait pas français mais il a progressé. Il a des difficultés en cours, n'arrive pas à suivre dans certaines matières, comme en technologie. " Je n'ai jamais utilisé le téléphone au pays. Quand on me dit de faire des choses avec l'ordinateur, c'est impossible! C'est difficile! "Sekou n'avait jamais étudié non plus les Sciences et Vie de la Terre. "Cela me pose beaucoup de problèmes ». "En anglais et en espagnol, je ne peux pas. Je connais quatre ou cinq langues dans mon pays. Là, j'arrive à apprendre le français. Je me débrouille. Mais l'anglais et l'espagnol en plus, je ne peux pas ".

Sékou est en quatrième mais aimerait arrêter le collège l'année prochaine. II envisage de travailler, en électricité. "Au foyer, on vit bien, on nous donne les habits, à manger. Mais j'ai besoin de choses aussi. Je ne peux pas continuer à aller à l'école jusqu'à 18 ans. Cela ne me plaît pas, j'aimerais travailler. " II voudrait pouvoir répondre seul à ses besoins. Les éducateurs lui disent que la prise en charge va s'arrêter à ses 18 ans et qu'ils ne pourront pas continuer à le soutenir. Il lui faut un apprentissage.

\section{Différences culturelles}

Chez lui, c'est un manque de respect de regarder l'adulte dans les yeux. " On ne parle pas trop avec un adulte ". Juste avec la famille mais on ne regarde pas dans les yeux! «Non !!! » Même pas les parents. On baisse la tête. II ne sait pas si l'on peut regarder sa femme dans les yeux. Les jeunes oui, mais pas les filles. 
Sekou s'est habitué, en France, à regarder les gens dans les yeux. Une fois, au début, un policier lui a dit qu'il mentait car il baissait les yeux. " Chez nous, c'est un manque de respect quand on regarde dans les yeux ".

Une autre différence culturelle qu'il constate, c'est que les jeunes ici ont le droit de parler quand un adulte les dispute. Ils peuvent s'expliquer. Ce n'est pas comme au Mali. II trouve cela bien et laissera ses enfants s'exprimer. "Tout le monde a le droit de parler $»$.

« Moi j'aimerais m'intégrer ici. Apprendre comment on vit ici, comment être un bon citoyen français. Cela va me dire que faire après. Avec l'école, on a commencé à apprendre beaucoup, beaucoup de choses. Surtout dans ce collège, on a des cours de FLE (Français Langue Etrangère) et on discute beaucoup avec elle. Les éducateurs aussi ici, ils nous aident beaucoup. Cela ne se fait pas, on ne dit pas ça... " "Tout est différent ici. II y a des choses que j'ai apprises ici. C'est beaucoup ! Je ne peux pas tout citer!»

"Par exemple, si quelqu'un te donne quelque chose ou te rend service, tu lui dis merci. Tu ne peux pas demander quelque chose sans dire s'il vous plaît. " Ces expressions de politesse existent aussi dans sa langue mais « on n'utilise pas; ici on utilise ». Son éducateur ne lui donne pas les choses s'il ne dit pas s'il te plaît. "Avec lui, on arrive à comprendre tout ça. On ne peut pas vivre avec quelqu'un si on ne le respecte pas, si on ne sait pas comment ça se passe. C'est intéressant de nous dire ça ".

Sekou n'a pas rencontré de situations où ses actes étaient mal perçus. "Moi je suis timide, je ne parle pas trop. J'ai peur de dire les choses qu'il ne faut pas dire. Sinon, je ne suis pas timide. Là, je m'amuse avec tout le monde. Avec les éducateurs, c'est pareil. Mais à l'école, je suis timide un peu. Je connais moins. Mais là, ça fait quatre mois. J'arrive à me faire des potes. "

Il évoque des différences. "Par exemple, au pays je n'allais pas à l'école. Là, ils m’ont mis à l'école. Ça j'aime bien. Là, il n'y a pas l'insécurité. Tu dors tranquille, il n'y a personne qui vient te taper. Tout est organisé en Europe, ici. Par exemple, j'étais malade, j'ai été opéré, j'ai été chez le docteur, je n'ai pas payé. Je ne sais pas comment c'est payé mais moi je n'ai pas payé avec mon argent. Là, tout va bien maintenant, je mange bien ici. Au pays, je n'avais pas tout ça! Ah là-bas, ce sont tes sous! Si tu es malade, tu vas guérir! Je n'ai jamais été à l'hôpital. Tu guéris tout seul. Il y a des médicaments que tu prends dans les arbres, tu coupes. ॥

\section{Intégration}

"Comme moi, on est quatre » dans tout le collège. Sekou explique que parmi les huit cents élèves de l'établissement, ils ne sont que quatre mineurs étrangers et ce 
serait les premiers! II ne croise pas forcément les autres qui n'ont pas les mêmes horaires. "Tu te trouves tout seul, Noir au milieu de huit cents élèves Français ! " II rit. "C'est dur, c'est dur. Mais on va y arriver quand même. C'est dur mais on va y arriver. ")

Sekou n'a pas trop de copains dans sa classe mais il a sympathisé avec des élèves d'autres niveaux, de la sixième à la troisième, qui viennent à lui quand il est seul. Au début, on venait lui poser des questions. "Tu vois dix élèves qui viennent te demander d'où tu viens. Ils étaient gentils. Mais ils ont arrêté. Maintenant, les choses commencent à changer un peu. Ils ne sont pas méchants mais ils ne s'imposent pas. C'est à nous d'aller les voir, de les faire parler mais eux ils ne viennent pas. " II ne sait pas pourquoi. "Chez nous, quand un jeune vient, on l'accueille. Ici, ils ne sont pas méchants mais je ne sais pas comment ça se passe. Tout le monde est dans sa maison. Chez nous, tout le monde est dehors. On se connaît. Ici, c'est différent. Quand tu viens ici pour t'intégrer dans les classes, c'est difficile. "

«Maintenant, c'est à nous d'aller les voir car ce n'est pas eux qui se sont déplacés. C'est nous qui nous sommes déplacés pour venir ici. Quand on arrive parmi les gens, on doit être comme eux. On ne peut pas être comme eux naturellement mais on doit apprendre avec eux. Par exemple, je ne veux pas être dans une classe et être toujours tout seul, rester dans ma culture et ne pas changer. J'aimerais être comme eux. Je ne peux pas changer ma peau mais j'aimerais être comme eux. La même culture. J'aimerais faire tout ce qu'ils font ".

Sekou a du mal à trouver des exemples pour expliquer en quoi il se sent différent. Il évoque les élèves qui se rassemblent, qui se font des copains. En revanche, quand lui rejoint un groupe, il a l'impression que l'on s'arrête un peu de parler. " J'aimerais être entre eux ". Il dit se sentir encore différent des autres. " Je ne suis pas totalement intégré. Je fais de mon mieux, je parle avec tout le monde. Certains ne veulent pas lui parler mais il les laisse. Le week-end, les autres lui disent qu'ils jouent aux jeux vidéo. Au foyer, ils n'en ont pas. Certains élèves se rendent aussi au McDonald's. Lui, il n'y est jamais allé à Langon et il n'a mangé qu'une fois à celui de Bordeaux.

\section{Exigences administratives}

Arrivé au mois de mars, il a été logé dans un hôtel durant deux mois par le département. Puis, l'âge déclaré a été contesté bien qu'il ait été évalué mineur. "J'ai été mis à la rue. Ils m'ont mis dehors " durant deux ou trois mois. II a déposé un recours en justice avec son avocat. Sachant qu'il avait des problèmes de santé, il a été opéré et hospitalisé, puis finalement mis à l'abri le temps de récupérer ses documents 
administratifs. Il a finalement bénéficié d'une prise en charge l'été dernier, qui l'a conduit au CAMINA ${ }^{49}$ de Langon.

Sekou reconnaît que ses documents ne sont pas complets. II manque des papiers. Son père va les refaire faire. "Ils m'ont donné six mois $d^{\prime} O P P^{50}$. Ma prise en charge était de six mois. Les six mois sont déjà passés. Ils m'ont demandé de faire envoyer mon extrait de naissance avec le jugement supplétif. J'ai envoyé le jugement supplétif mais ils ont dit que ce n'était pas complet. Cela fait deux ou trois mois. J'appelle mes parents; chez nous le jugement supplétif existe mais quand il arrive ici, on nous dit que ce n'est pas bon. Là, il y a des difficultés. "

" Ici, quand on est mineur, on te prend en charge ou je ne sais pas quoi. Mais il faut des preuves. C'est ça que j'ai compris. C'est bizarre ! On ne peut pas quitter chez nous et venir ici... Moi, j'aimerais avoir 25 ans en fait, 30 ans comme ça, profiter de pouvoir tout faire tout seul! Là où je suis, par exemple au foyer, je n'aimerais pas y être toute la journée. II y a des gens qui te disent de faire ça ou de ne pas faire ça. Si je pouvais choisir une vie, un âge, ce ne serait pas 15 ou 16 ans. Ça ne serait pas ça. C'est comme une prison, moi je considère ça comme une prison. Il y a des règles. En Afrique, il n'y a pas cela. Même si tu vis avec tes parents, tu sors à I'heure que tu veux, tu rentres à l'heure que tu veux, même si tu as 5 ou 6 ans. Tu as grandi comme ça. Quand on arrive ici, on nous dit de ne pas sortir après $22 \mathrm{~h} 00$. Et chaque jour à l'école. C'est difficile. Si j'avais choisi un âge ici, je n'aurais pas pris 15 ou 16 ans. »

«Moi je trouve que ce n'est pas logique mais c'est leur boulot. On ne peut pas dire, mais ce n'est pas logique. Tu ne peux pas venir ici et mentir, dire que tu as 15 ans, cela n'a rien à voir en fait. Tu peux dire que tu as 25 ans et rester en France ! Cela n'a rien à voir de mentir sur ton âge, l'âge que tu vas garder jusqu'à la mort ! Cela n'a rien à voir en fait $"$.

La chef de service lui a expliqué beaucoup de choses, sur les prises en charge à la minorité par exemple. Mais Sekou a un copain qui a demandé l'asile à Bordeaux et qui perçoit une allocation, que lui n'a pas. II a même son appartement tout seul ! Lui, il n'aurait pas menti pour venir ici. II ne comprend pas. " Mais c'est leur droit aussi de ne pas nous comprendre. " Personne ne lui a jamais dit avoir menti sur son âge. "Je ne peux pas croire ça même ». "Ça peut, mais je ne crois pas ». Le juge lui a parlé de ces situations mais il n'est pas d'accord avec lui. "Dans un foyer, non. Chaque jour à l'école non. Je ne peux pas comprendre ça ".

Sait-il ce que signifie la protection ? « Je ne comprends pas. La protection, cela me dit quelque chose ". II revient sur son copain, adulte, majeur, qui a obtenu l'asile et est protégé aussi. Même si on lui donnait des millions pour vivre dans un foyer avec

\footnotetext{
${ }^{49}$ Carré d'Accueil pour les Mineurs Non Accompagnés.

${ }^{50}$ Ordonnance de Placement Provisoire.
} 
des jeunes de 10 ans, il ne pourrait pas. II compare avec le collège et les classes d'âge. "Vivre avec quelqu'un qui n'a pas le même âge, un jour, neuf mois ou dix mois, ensemble, tu ne peux pas tenir, tu ne peux pas! Moi, c'est ce que je trouve ». Dans sa classe, il y en a qui sont plus jeunes, qui ont 13 ou 14 ans et cela ne le gêne pas. Mais pas avec des élèves de 10 ans ! " Je ne peux pas! " " Je ne vois pas l'intérêt de changer ton âge ».

Ce qui le touche en ce moment, c'est qu'en allant à Paris faire les démarches consulaires, ils n'ont pas été bien accueillis et ils n'ont pas pu tout faire. "On est les mêmes mais eux ils n'arrivent pas à faire ». Bien qu'ils aient parlé dans leur langue, ils n'ont pas été reçus. II ne comprend pas. Alors qu'il y a des français qui « font tout pour nous ". Cela le touche de ne pas avoir ses papiers.

Sa prise en charge a été renouvelée jusqu'en avril. S'il n'a pas le jugement supplétif, elle prendra fin. "Ça m'inquiète ». Il connait un jeune camerounais qu'ils ont " mis dehors " et qui vit chez son patron maintenant. Et si cela s'arrête pour lui ? " Je ne veux pas que ça s'arrête mais si ça s'arrête, je vais retourner à Bordeaux parce qu'ici, il n'y a pas de squats. Je ferai encore des recours. »

\section{Soutiens}

Quand Sekou a besoin, il va voir la chef de service. "Je m'entends bien avec elle ". II n'y avait personne pour fêter son anniversaire dimanche dernier et les éducateurs n'étaient même pas au courant. II ne l'a toujours pas célébré ! La chef de service lui a proposé de le faire demain.

Quand il n'a pas le moral, c'est elle aussi qu'il va voir. Avec les autres, il s'entend bien mais " on ne peut pas se comprendre. " "Avec elle, on se comprend bien. Avec les éducateurs, on ne se comprend pas». Alors que lui pense avoir raison, les éducateurs considèrent qu'il a tort. "Julia quand je la vois, ce n'est pas ma maman mais je la vois comme une maman. Elle peut me dire des choses, d'arrêter ça... Elle est gentille. Je respecte tout le monde mais je lui dis les choses à elle. Je l'écoute plus. C'est elle qui m'a fait comprendre beaucoup de choses. Quand elle me parle, je comprends vite ". Le fait qu'elle soit chef de service ne change rien. "Moi je considère Julia comme Julia mais chef de service non. Je ne vois pas ça. " "J'ai confiance en elle " et en une autre femme à Paris, une Malienne qui l'a accueilli à son arrivée. II a passé un mois chez elle. Mais elle n'a pas pu le garder quand elle a su qu'il était mineur. Il était entré en France avec le petit frère de cette dame.

Fait-il confiance aux adultes? "Aux adultes, non! En France ici, non! » Mis à part cette dame et Julia. Au Mali, " pas trop " non plus. En France, il ne connait pas bien les adultes. Envers les jeunes du service, il a confiance. Mais les personnes plus âgées, il ne veut pas les déranger. 


\section{5. ÊTRE ADULTE}

\section{Question de maturité}

Il y a des choses que les autres connaissent plus que lui. "Eux, ils me disent des histoires que je n'ai jamais entendues". II évoque certaines matières comme la technologie, dans lesquelles "ils connaissent tout, plus que moi. " "Dans la vie, même, ils connaissent plus que moi. Mais j'ai vécu des choses qu'eux n'ont pas vécues. Par exemple, moi je peux dire que je suis né dans une maison mais que j'ai grandi dans la rue. Au Maroc, c'était pire, en Mauritanie pareil... Je peux essayer de comprendre des choses mais eux ils ne peuvent pas comprendre. On dit que quelqu'un qui a fait dix capitales connait plus que celui qui a fait dix ans d'école. Eux, ils peuvent apprendre à l'école, mais ils ne voient pas. C'est ça que j'ai compris. »

Les éducateurs disent souvent aux jeunes du foyer qu'ils sont plus mâtures que les Français et il entend le même discours d'autres personnes en ville. Ils se fondent sur la taille, alors qu'il y a aussi des Français qui sont grands ou plus costauds que lui. " Je ne suis pas plus mâture qu'eux! " II justifie son propos par le fait que les autres élèves réussissent mieux à faire les devoirs, à rédiger. Ils y arrivent directement, le font vite. Pour lui, c'est une preuve de maturité.

Sekou a certains copains plus jeunes mais qui connaissent plus de choses que lui. II parle d'une amie âgée de 12 ans qui lui dit ce qu'il ne doit pas faire. "Elle est toute petite mais c'est elle qui me donne des conseils. Alors qu'elle n'a que 12 ans ! » II ne sait pas pourquoi elle est mûre mais il pense que c'est du fait de l'éducation.

Sekou compare l'éducation reçue dans les familles et au foyer où il y a certes des éducateurs, mais pas toute la journée. II ne les voit que ponctuellement. "On est éduqués mais pas comme ceux qui habitent avec les parents. " Dans la journée, il n'a personne qui peut lui dire ce qu'il fait de bien ou pas, s'il n'a ni les éducateurs ni l'école, alors que les autres ont leurs parents, "matin, midi et soir ». Un parent ne peut pas dire à son fils de faire des bêtises. "Ils ont ça mais moi je n'ai pas ça ".

"Les éducateurs, on ne se voit pas trop en fait. lls peuvent me voir mais on ne reste pas ensemble. Ils sont là quand j'ai besoin d'aller à l'hôpital ou de faire mes courses" ".

Il revient sur son amie. "Elle me dit des choses que je n'ai jamais entendues. Je n'aurais jamais imaginé qu'à 12 ans on pouvait dire ça ». II donne l'exemple d'une fois où elle l'a vu fumer. Elle ne lui parlait plus, expliquant que la cigarette tuait et gaspillait l'argent. II n'avait pas pensé à cela.

\section{La majorité}

Dans certains domaines, Sekou ne peut pas penser comme un petit car il n'a pas grandi avec ses parents et doit "se débrouiller » pour avoir les choses. II se dit lui- 
même qu'il ne doit pas agir de telle ou telle manière, n'a personne pour le conseiller. " Je suis tout seul».

Se considère-t-il comme un enfant, un adolescent ou un adulte ? II demande ce qu'est un adolescent. Après avoir écouté l'explication, il répond être un adolescent. II ne se considère pas comme un adulte. "Je ne suis pas un enfant non plus ". Au Mali, on ne parle pas d'adolescent. "Tu es un enfant jusqu'à 5 ans, 6 ans, après cela s'arrête là. Tu te débrouilles. II y a des enfants qui travaillent depuis leurs 3 ans. Ce ne sont pas des adultes mais tu travailles, tu fais la même chose que les adultes. »

La majorité, "cela existe mais pas trop, pas comme ici. " Si la famille a de l'argent, les parents s'occupent de leurs enfants même jusqu'à leurs 30 ou 40 ans. Sinon, il faut se débrouiller.

Le fait de signer seul les papiers à 18 ans ? "Chez nous, il n'y a même pas de papiers ". II ne sait pas si la majorité existe dans les grandes villes car il ne connaît que la vie en village et ne peut pas dire non plus à quel âge on est adulte au Mali. Il ne s'est pas dit qu'il était adulte quand il est parti. Mais il savait qu'il devrait se débrouiller seul. " À l'école coranique, tu sais quel est ton travail, ce qu'il faut faire. II n'y pas d'adultes ou quoi que ce soit... »

Avoir 18 ans ici, il comprend " un peu mais pas trop ". Il a compris que quand on est petit ici, on n'a pas beaucoup de " pouvoir ». Ses copains ne peuvent pas aller le voir si les parents refusent. II ne comprend pas trop mais n'a pas plus d'avis sur cette question.

\section{Sa vie dans dix ans}

Plus tard, Sekou voudrait vivre à Langon. "J'aime Langon. Je connais, je maîtrise un peu. Je me déplace tout seul. Au début, c'était difficile, mais maintenant, j’y arrive. " "J'aimerais avoir du travail d'abord. Avant tout, c'est le travail. Avoir mon appart aussi. »

Envisage-t-il de repartir au Mali ? "Au mali non! Pas du tout non ! Pour les vacances j'aimerais y aller un peu mais retourner là-bas, non ! Je n'aime pas ! Je n'aime pas du tout! J'aime le Mali mais avec la vie que j'ai vécue là-bas, je ne voudrais pas retourner là-bas et faire ça encore. Parce que j'ai donné ma vie à la mort une fois. Je suis passé par l'eau. Quand tu passes par l'eau, tu as donné ta vie à la mort. Tu ne sais pas si tu vas remonter. "

« J'ai toujours pensé que ça serait difficile. Je n'arrive pas à comprendre mais je me suis dit dans ma tête que tout serait difficile. Ce que j'ai vécu a toujours été difficile jusqu'à présent. Je ne peux pas imaginer autre chose. » 